\title{
Sinais de Produção de Novos Bósons Vetoriais no LHC
}

\author{
Dorival Gonçalves Netto \\ Orientador: Prof. Dr. Oscar J. P. Éboli
}

Dissertação apresentada ao Instituto de Física da Universidade de São Paulo para a obtenção do título de Mestre em Ciências.

Comissão examinadora:

Prof. Dr. Oscar J. P. Éboli (IF-USP)

Prof. Dr. Orlando L. G. Peres (Unicamp)

Prof. Dr. Rogério Rosenfeld (IFT-UNESP)

São Paulo

2009 


\section{FICHA CATALOGRÁFICA}

Preparada pelo Serviço de Biblioteca e Informação do Instituto de Física da Universidade de São Paulo

Gonçalves Netto, Dorival

Sinais de produção de novos bósons vetoriais no LHC - São Paulo, 2009.

Dissertação (Mestrado) - Universidade de São Paulo. Instituto de Física, Departamento de Física Matemática

Orientador: Prof. Dr. Oscar José Pinto Éboli

Área de Concentração: Física

Unitermos: 1. Física de partículas; 2. Física além do modelo padrão. 


\section{Resumo}

Neste trabalho realizamos uma abordagem fenomenológica da observação de novos bósons de spin-1 associados ao setor de Quebra de Simetria Eletrofraca. Como motivação a essa análise estudamos primeiramente o caso especial de modelos baseados no mecanismo de Quebra espontânea de Simetria por Condições de Contorno, os quais também apresentam uma torre de bósons vetoriais de Kaluza-Klein assegurando a unitariedade no espalhamento entre bósons gauge.

$\mathrm{Na}$ análise fenomenológica efetuamos uma abordagem independente de modelo analisando o potencial do Large Hadron Collider (LHC) na detecção de novos bósons vetoriais associados ao setor de quebra de simetria. Para tal estudamos os processos $p p \rightarrow l^{ \pm} j j \mathbb{E}_{T}, l^{+} l^{\prime-} \mathbb{E}_{T}$, $l^{\prime \pm} l^{+} l^{-} E_{T}$ e $l^{+} l^{-} j j\left(l, l^{\prime}=e, \mu\right.$ e $j=$ jato) obtendo que o LHC possui um grande potencial de descoberta ou exclusão dessa classe de extensões ao Modelo Padrão. 


\section{Abstract}

In this work we performed a phenomenological observation of new spin-1 bosons associated with the Electroweak Symmetry Breaking sector. As motivation for this analysis we previously studied the special case of models based on the mechanism of Eletroweak Symmetry Breaking via Boundary Conditions, which also have a tower vector of Kaluza-Klein bosons ensuring unitarity in scattering between gauge bosons.

In the phenomenological analysis we performed a model independent approach to analyzing the potential of the Large Hadron Collider (LHC) in the detection of new vector bosons associated with the symmetry breaking sector. For this we studied the processes $p p \rightarrow l^{ \pm} j j \mathbb{E}_{T}$, $l^{+} l^{\prime-} E_{T}, l^{\prime \pm} l^{+} l^{-} E_{T}$ and $l^{+} l^{-} j j\left(l, l^{\prime}=e, \mu\right.$ e $j=$ jets $)$ getting that the LHC has great potential for discovery or exclusion of this class of extensions to the Standard Model. 


\section{Agradecimentos}

Ao Prof. Oscar Éboli, pela orientação e pelo grande incentivo.

À Fundação de Amparo à Pesquisa do Estado de São Paulo (FAPESP) pela bolsa concedida.

À todos os meus grandes amigos que fiz durante esses anos de universidade, em especial à Ricardo e Everton companheiros sempre presentes durante todo o mestrado.

Aos meus pais Selma e Dorival e a minha irmã Ana Carolina, à todos eles o meu amor sempre incondicional. 


\section{Sumário}

1 Introdução $\quad 8$

2 O Modelo Padrão das Partículas Elementares $\quad 10$

2.1 O Modelo Padrão . . . . . . . . . . . . . . . . . . . . . . . 10

2.1.1 Interações Fortes (QCD) . . . . . . . . . . . . 15

2.1.2 Interações com Correntes Carregadas . . . . . . . . . . . 15

2.1.3 Interações com Correntes Neutras . . . . . . . . . . 16

2.1.4 Interação entre os bósons de gauge eletrofracos . . . . . . . 17

2.2 Quebra Espontânea de Simetria . . . . . . . . . . . . . . . . 19

2.2.1 O Teorema de Goldstone . . . . . . . . . . . . 19

2.2.2 O Mecanismo de Higgs . . . . . . . . . . . 20

2.2.3 Massa para os férmions e a matriz CKM . . . . . . . . 23

2.2.4 As interações do Higgs . . . . . . . . . . . . . . . 26

2.3 Simetria Custodial . . . . . . . . . . . . . . 26

2.4 Resultados experimentais . . . . . . . . . . . . . . 29

2.5 Limites teóricos na massa do Higgs . . . . . . . . . . . . . . . 32

2.5.1 Unitariedade . . . . . . . . . . . . . . 32

2.5.2 Trivialidade e Estabilidade do Vácuo . . . . . . . . . . . 35

2.6 O Problema da Hierarquia do Modelo Padrão . . . . . . . . . . . 37

3 Quebra espontânea de simetria por condições de contorno $\quad 40$

3.1 Teorias de gauge em uma dimensão extra compacta: fixação de gauge e condições de contorno . . . . . . . . . . . . . . . 40

3.1.1 A origem da massa para os KK's . . . . . . . . . . . 45

3.2 Teorias de Gauge com escalares nas branas . . . . . . . . . . . 46

3.3 Toy models . . . . . . . . . . . . . . . . . . . . . 48

3.3.1 Geração de massa para o fóton . . . . . . . . . . . 48

3.3.2 Geração de massa para os bósons de $S U(2) \ldots . .49$ 
3.3.3 Higgsless model com simetria custodial . . . . . . . . . . . 51

3.4 Unitariedade e os modos de Kaluza-Klein . . . . . . . . . . . . . 54

4 Sinais de Produção de Novos Bósons Vetoriais no LHC 60

4.1 Estrutura da análise . . . . . . . . . . . . . . . . 62

4.2 Análise dos cortes . . . . . . . . . . . . . . 65

4.2.1 $p p \rightarrow Z^{\prime} \rightarrow W^{+} W^{-} \rightarrow l^{ \pm} j j \mathbb{E}_{T} \ldots \ldots \ldots 65$

$4.2 .2 \quad p p \rightarrow Z^{\prime} \rightarrow W^{+} W^{-} \rightarrow l^{+} l^{\prime-} \mathbb{E}_{T} \ldots \ldots \ldots \ldots . \ldots . \ldots . \ldots 71$

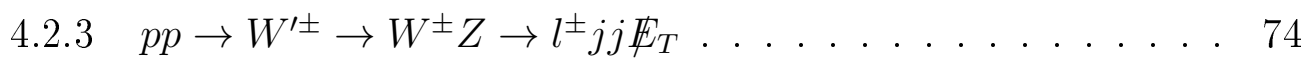

4.2.4 $p p \rightarrow W^{ \pm} \rightarrow W^{ \pm} Z \rightarrow l^{ \pm} l^{+} l^{-} \mathbb{E}_{T} \ldots \ldots \ldots . \ldots . \ldots 75$

4.2.5 $\quad p p \rightarrow W^{ \pm} \rightarrow W^{ \pm} Z \rightarrow l^{+} l^{-} j j \ldots \ldots \ldots . \ldots . \ldots 77$

4.3 Observabilidade do sinal . . . . . . . . . . . . . . 79

4.4 Conclusões . . . . . . . . . . . . . . . . . . 83

5 Conclusão $\quad 84$ 


\section{Lista de Figuras}

2.1 Potencial da lagrangeana (2.41) no caso (a) com $\mu^{2}>0$ e no caso (b) com $\mu^{2}<0$, onde temos a quebra espontânea da simetria. . . . 20

2.2 Comparação entre os resultados experimentais e o melhor ajuste global ao MP, ref. [19]. . . . . . . . . . . . . . 30

$2.3 \triangle \chi^{2}=\chi^{2}-\chi_{\min }^{2}$ em função da massa do Higgs obtido através de um ajuste global dos dados do LEP, SLC e Tevatron. A faixa colorida vertical indica regiões com $95 \%$ confiança de exclusão através de buscas diretas no LEP e Tevatron, ref. [19]. . . . . . . . . . . . . . 31

2.4 Diagramas que contribuem ao espalhamento $\omega^{+} \omega^{-} \rightarrow \omega^{+} \omega^{-}$. . . . 33

2.5 Regiões permitidas à $M_{H}$ em função do cutoff da teoria $\Lambda$. Nos extremos superior e inferior em preto temos os limites de Trivialidade e Estabilidade respectivamente. Nas partes em vermelho temos regiões de ajuste fino de $1 \%$ e $10 \%$ como indicado no gráfico. A região em branco é consistente com um ajuste fino menor que $10 \%$, ref. [18]. . . . . . . . . . . . . . . 39

3.1 Espectro de massa dos modos de Kaluza-Klein. . . . . . . . . . . . 44

3.2 Quebra da simetria $S U(2) \rightarrow U(1)$ por condições de contorno. . . . 50

3.3 Quebra de simetria por condições de contorno no Higgsless model com dimensão extra plana, ref. [11]. . . . . . . . . . . . . . . 51

3.4 Cutoff da teoria sem Higgs com métrica plana em função da massa do primeiro bóson vetorial excitado, ref. [30]. . . . . . . . 58

4.1 Diagramas de produção de $Z^{\prime}$ e $W^{\prime}$ via fusão de bósons eletrofracos. 61

4.2 Diagramas de produção de $Z^{\prime}$ e $W^{\prime}$ via processos Drell Yan. . . . . 61

4.3 Diagrama do sinal $p p \rightarrow Z^{\prime} \rightarrow W^{+} W^{-} \rightarrow l^{ \pm} j j \mathbb{E}_{T}, \operatorname{com} l^{ \pm}=e^{ \pm}, \mu^{ \pm} .65$ 
4.4 Distribuições do momento transversal do jato menos energético $p_{T}^{j \min }$, do jato mais energético $p_{T}^{j \max }$, do lépton carregado $p_{T}^{l}$ e da energia faltante $\mathbb{E}_{T}$ para o background total e sinal $p p \rightarrow Z^{\prime} \rightarrow l^{ \pm} j j \mathbb{F}_{T}$. O histograma em vermelho corresponde ao background e o azul ao sinal com $M_{Z^{\prime}}=0.5 \mathrm{TeV}$ e largura $\Gamma_{Z^{\prime}}=0.05 M_{Z^{\prime}}$. O histograma $p_{T}^{j \text { min }}$ possui apenas os cortes básicos (4.18), o histograma $p_{T}^{\text {jmax }}$ possui além dos cortes básicos o corte em $p_{T}^{j \text { min }}$ tabela $(4.2)$, o $p_{T}^{l}$ possui todos os cortes anteriores e o em $p_{T}^{j \max }$, por fim o $\mathbb{E}_{T}$ possui todos os anteriores e o em $p_{T}^{l} \ldots \ldots \ldots$. . . . . . . 68

4.5 Distribuição para a massa invariante reconstruída para o processo $p p \rightarrow j j l^{ \pm} \mathbb{E}_{T}$ utilizando o menor valor de momento longitudinal para o neutrino. Com sinal $M_{Z^{\prime}}=0.5 \mathrm{TeV}$ no painel da esquerda e $1.0 \mathrm{TeV}$ no da direita sobre os respectivos backgrounds hachurados, após os cortes $(4.18)-(4.20)$. . . . . . . . . . . . 69

4.6 Distribuições para $\Delta R_{j j}$ do sinal com estado final $l^{ \pm} j j E_{T}$ para diferentes valores de $M_{Z^{\prime}}$ no caso ilustrativo $\Gamma_{Z^{\prime}}=0.05 M_{Z^{\prime}}$. . . . 70

4.7 Diagrama do sinal $p p \rightarrow Z^{\prime} \rightarrow W^{+} W^{-} \rightarrow l^{ \pm} l^{\prime \pm} \mathbb{E}_{T}$, com $l^{ \pm}, l^{ \pm}=$

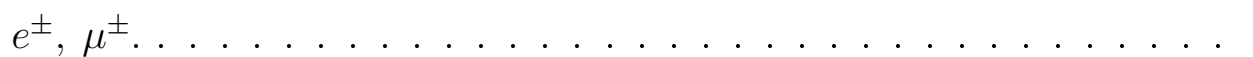

4.8 Distribuição angular azimutal da separação entre os léptons carregados do estado final $l^{+} l^{\prime-} \mathbb{E}_{T}$. A linha sólida representa a distribuição do sinal para $M_{Z^{\prime}}=0.5 \mathrm{TeV}$ com $\Gamma_{Z^{\prime}} / M_{Z^{\prime}}=0.1$ e a tracejada está associada ao background $t \bar{t}$. . . . . . . . . . . . . 72

4.9 Diagrama do sinal $p p \rightarrow W^{ \pm} \rightarrow W^{ \pm} Z \rightarrow l^{ \pm} j j \mathbb{E}_{T}$, com $l^{ \pm}=e^{ \pm}, \mu^{ \pm} . \quad 74$

4.10 Diagrama do sinal $p p \rightarrow W^{ \pm \pm} \rightarrow W^{ \pm} Z \rightarrow l^{+} l^{-} l^{\prime \pm} \mathbb{E}_{T}, \operatorname{com} l^{ \pm}, l^{ \pm \pm}=$

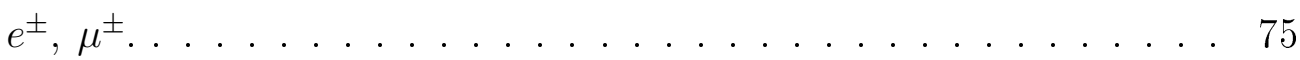

4.11 Diagrama do sinal $p p \rightarrow W^{ \pm} \rightarrow W^{ \pm} Z \rightarrow l^{+} l^{-} j j$, com $l^{ \pm}=e^{ \pm}, \mu^{ \pm} . \quad 77$

4.12 Distribuição para a massa invariante reconstruída para o processo $p p \rightarrow l l^{-} j j$ com sinal $M_{W^{\prime}}=0.5 \mathrm{TeV}$ no painel da esquerda e $1.0 \mathrm{TeV}$ no da direita sobre os respectivos backgrounds hachurados, após os cortes $(4.32)-(4.34)$. . . . . . . . . . . . 78

4.13 As regiões hachuradas referem-se ao intervalo de observação de um $Z^{\prime}$ com massas $M_{Z^{\prime}}=0.5,1.0$ e $1.5 \mathrm{TeV}$ com pelo menos $5 \sigma$ de significância na reação $p p \rightarrow Z^{\prime} \rightarrow W^{+} W^{-} \rightarrow l^{ \pm} j j \mathbb{E}_{T} . \ldots . . .80$

4.14 As regiões hachuradas referem-se ao intervalo de observação de um $Z^{\prime}$ com massas $M_{Z^{\prime}}=0.5,1.0$ e $1.5 \mathrm{TeV}$ com pelo menos $5 \sigma$ de significância na reação $p p \rightarrow Z^{\prime} \rightarrow W^{+} W^{-} \rightarrow l^{+} l^{-} \mathbb{E}_{T} . \ldots . .81$ 
4.15 As regiões hachuradas referem-se ao intervalo de observação de um $W^{\prime}$ com massas $M_{W^{\prime}}=0.5,1.0$ e $1.5 \mathrm{TeV}$ com pelo menos $5 \sigma$ de significância na reação $p p \rightarrow W^{ \pm} \rightarrow W^{ \pm} Z \rightarrow l^{ \pm} j j \mathbb{E}_{T} . \quad \ldots . . .82$

4.17 As regiões hachuradas referem-se ao intervalo de observação de um $W^{\prime}$ com massas $M_{W^{\prime}}=0.5,1.0$ e $1.5 \mathrm{TeV}$ com pelo menos $5 \sigma$ de significância na reação $p p \rightarrow W^{ \pm} \rightarrow W^{ \pm} Z \rightarrow l^{+} l^{-} j j . \ldots$. . . . . 82

4.16 As regiões hachuradas referem-se ao intervalo de observação de um $W^{\prime}$ com massas $M_{W^{\prime}}=0.5,1.0$ e $1.5 \mathrm{TeV}$ com pelo menos $5 \sigma$ de significância na reação $p p \rightarrow W^{ \pm} \rightarrow W^{ \pm} Z \rightarrow l^{ \pm \pm} l^{+} l^{-} \mathbb{E}_{T} . \ldots . .83$ 


\section{Lista de Tabelas}

2.1 Campos de matéria do Modelo Padrão com seus respectivos números quânticos. . . . . . . . . . . . . . . . 12

2.2 Acoplamentos vetorial e axial dos quarks e léptons com o $Z^{0} \ldots$. . 17

4.1 Smearing na energia $E(\mathrm{em} \mathrm{GeV})$ com erro Gaussiano. . . . . . . . 64

4.2 Cortes para o $p p \rightarrow Z^{\prime} \rightarrow W^{+} W^{-} \rightarrow l^{ \pm} j j \mathbb{E}_{T}$ em função da massa do $Z^{\prime}$. . . . . . . . . . . . . . . . . . 67

4.3 Seção de choque para o sinal $Z^{\prime}$ e background em fb para o estado final $j j l^{ \pm} \mathbb{E}_{T}(l=e, \mu)$ após os cortes (4.18) - (4.22). Nesses resultados ainda não foram incluídos as eficiências experimentais de reconstrução de jatos e léptons. Para o sinal apresentamos os resultados para o valor ilustrativo $\Gamma_{Z^{\prime}}=0.05 M_{Z^{\prime}} \ldots \ldots \ldots 70$

4.4 Seção de choque para o sinal $Z^{\prime}$ e background em fb para o estado final $l^{+} l^{\prime-} \mathbb{E}_{T}\left(l, l^{\prime}=e, \mu\right)$. Para léptons de mesmo sabor foram aplicados os cortes (4.23) - (4.25), já para léptons de sabores distintos (4.23) - (4.28). Nesses resultados ainda não foram incluídas as eficiências experimentais de reconstrução de jatos e léptons. Para o sinal apresentamos os resultados para o valor ilustrativo $\Gamma_{Z^{\prime}}=0.05 M_{Z^{\prime}}$.

4.5 Seção de choque para o sinal $W^{\prime}$ e background em fb para o estado final $j j l^{ \pm} \mathbb{E}_{T}(l=e, \mu)$ após os cortes (4.18) - (4.22) trocando $M_{W}$ por $M_{Z}$ em (4.20) e $M_{Z^{\prime}}$ por $M_{W^{\prime}}$ em (4.22). Nesses resultados ainda não foram incluídas as eficiências experimentais de reconstrução de jatos e léptons. Para o sinal apresentamos os resultados para o valor ilustrativo $\Gamma_{W^{\prime}}=0.05 M_{W^{\prime}} \ldots \ldots \ldots \ldots \ldots \ldots$ 
4.6 Seção de choque para o sinal e background com estado final $l^{\prime \pm} l^{+} l^{-} \mathbb{E}_{T}$ $\left(l, l^{\prime}=e, \mu\right)$ após os cortes (4.29) -(4.31). $\sigma_{M P}$ denota a soma das contribuições do background redutível e irredutível. Para o sinal apresentamos os resultados para o valor ilustrativo $\Gamma_{W^{\prime}}=0.05 M_{W^{\prime}}$.

4.7 Sinal do $W^{\prime}$ e background do MP para o estado final $l^{+} l^{-} j j$ com $l^{ \pm}=e^{ \pm}, \mu^{ \pm}$após os cortes (4.32) - (4.35). Nesses resultados ainda não foram incluídas as eficiências experimentais de reconstrução de jatos e léptons. Para o sinal apresentamos os resultados para o valor ilustrativo $\Gamma_{W^{\prime}}=0.05 M_{W^{\prime}} \ldots \ldots \ldots \ldots \ldots$. . . . . . . 79 


\section{Capítulo 1}

\section{Introdução}

O Modelo Padrão das Partículas Elementares é uma teoria que tem sido objeto de intenso escrutíneo experimental. Tendo suas predições paulatinamente confirmadas passando pela descoberta das correntes neutras na década de 70, pela produção direta de $W^{ \pm}$e $Z$ na década de 80 aos dias de hoje em que efetuamos testes de precisão indicando um incrível acordo entre os resultados experimentais e as predições do modelo.

Todavia o setor de quebra espontânea de simetria desse modelo ainda carece de maiores confirmações experimentais, dado que o seu ingrediente fundamental uma partícula escalar denominada Higgs, ainda não foi descoberta. Além disso temos que sua existência nos colocaria diante de um problema iminente, pois sua massa possui correções radiativas dependendo quadraticamente do cutoff da teoria, implicando em um ajuste fino não natural desse parâmetro já na escala de $1 \mathrm{TeV}$. Esse problema, denominado Problema da Hierarquia de Escalas, é um dos maiores indícios de uma possível observação de nova física no Large Hadron Collider (LHC), um acelerador de partículas tipo $p p$ construído no CERN com energia do centro de massa de $\sqrt{s}=14 \mathrm{TeV}$.

Almejando contornar esse ajuste fino um conjunto bastante amplo de teorias foi proposto ao longo dos últimos anos. Uma das propostas mais recentes são os Higgsless Models [21, 24], onde a quebra da simetria ocorre por condições de contorno em uma dimensão extra compacta, e a unitariedade no espalhamento dos bósons vetoriais $W$ e $Z$ é assegurada pela existência de torres de bósons vetoriais massivos.

Para obtermos um melhor entendimento dessas questões efetuamos no capítulo 2 deste trabalho uma abordagem sobre os aspectos fundamentais do Modelo Padrão 
analisando suas interações, o mecanismo de quebra espontânea de simetria, os limites teóricos e experimentais na massa do Higgs e o Problema da Hierarquia de Escalas.

No capítulo 3 expusemos uma possível solução ao problema da Hierarquia, a saber, o mecanismo de Quebra Espontânea de Simetria por Condições de Contorno. Mostramos suas características fundamentais e quais os requisitos que as teorias que o utilizam devem satisfazer para ser uma possível Teoria Além do Modelo Padrão.

Por fim, efetuamos uma análise independente de modelo, no capítulo 4, para os sinais de produção de novos bósons vetoriais no LHC. Os quais são previstos, por exemplo, pelas teorias com Quebra Espontânea de Simetria por Condições de Contorno. Nessa análise escolhemos 5 canais de produção dos novos bósons vetoriais, expressos nas equações (4.1) e (4.2), e apontamos o espaço de parâmetros que é passível de observação no LHC com uma luminosidade integrada de $100 \mathrm{fb}^{-1}$. 


\section{Capítulo 2}

\section{O Modelo Padrão das Partículas Elementares}

"It doesn't matter how beautiful your theory is, it doesn't matter how smart you are. If it doesn't agree with experiment, it's wrong"

Richard P. Feynman

\subsection{O Modelo Padrão}

O Modelo Padrão (MP) é uma teoria de unificação das forças forte, fraca e eletromagnética onde estas originam-se da troca de bosons de gauge de spin 1 entre as partículas que constituem a matéria. Os bósons de gauge eletrofracos estão associados ao grupo de simetria $S U(2)_{L} \otimes U(1)_{Y}$ e os das interações fortes ao grupo $S U(3)_{c}[1,2,3,4]$.

O Modelo Padrão possui dois tipos de campos:

- Bósons de Gauge: Estes bósons estão associados aos geradores do grupo de simetria do MP

$$
S U(3)_{c} \otimes S U(2)_{L} \otimes U(1)_{Y}
$$

Associadas à interação forte temos 8 partículas $\mathrm{G}_{\mu}^{1 \ldots 8}$ não massivas denominadas glúons, que correspondem ao subgrupo $S U(3)_{c}$. O índice "c" remete a "cor". Toda partícula que transforma em relação a essa parte do grupo de gauge se acopla aos glúons e possui um índice de cor. Seus 8 geradores satisfazem as seguintes propriedades:

$$
\left[T^{a}, T^{b}\right]=i f^{a b c} T^{c}, \quad \operatorname{tr}\left(T^{a} T^{b}\right)=\frac{1}{2} \delta^{a b},
$$


onde $f^{a b c}$ é o tensor de estrutura do grupo e os geradores na representação fundamental são associados às matrizes de Gell-Mann $\left(\lambda^{a}\right)$ por

$$
T^{a}=\frac{\lambda^{a}}{2}
$$

No setor eletrofraco temos 1 bóson $B_{\mu}$ associado subgrupo $U(1)_{Y}$, que corresponde ao gerador $Y$ (hipercarga), e 3 bosons de gauge $W_{\mu}^{1 \ldots 3}$ associados ao fator $S U(2)_{L}$ cujos geradores $I^{a}$ satisfazem ${ }^{1}$

$$
\left[I^{a}, I^{b}\right]=i \epsilon^{a b c} I^{c}, \quad\left[I^{a}, Y\right]=0,
$$

possuindo a seguinte representação fundamental:

$$
I^{a}=\frac{\sigma^{a}}{2} ; \quad \sigma^{1}=\left(\begin{array}{cc}
0 & 1 \\
1 & 0
\end{array}\right), \quad \sigma^{2}=\left(\begin{array}{cc}
0 & -i \\
i & 0
\end{array}\right), \quad \sigma^{3}=\left(\begin{array}{cc}
1 & 0 \\
0 & -1
\end{array}\right)
$$

Associados a cada fator do grupo temos os seguintes tensores de intensidade

$$
\begin{aligned}
G_{\mu \nu}^{a} & =\partial_{\mu} G_{\nu}^{a}-\partial_{\nu} G_{\mu}^{a}+g_{s} f^{a b c} G_{\mu}^{b} G_{\nu}^{c} \\
W_{\mu \nu}^{a} & =\partial_{\mu} W_{\nu}^{a}-\partial_{\nu} W_{\mu}^{a}+g \varepsilon^{a b c} W_{\mu}^{b} W_{\nu}^{c} \\
B_{\mu \nu} & =\partial_{\mu} B_{\nu}-\partial_{\nu} B_{\mu},
\end{aligned}
$$

onde $g_{s}, g$ e $g^{\prime}$ são respectivamente as constantes de acoplamento dos grupos $S U(3)_{c}, S U(2)_{L}$ e $U(1)_{Y}$.

- Campos de Matéria: Esse conjunto é constituído por partículas fundamentais de spin $\frac{1}{2}$. As interações desses férmions podem ser resumidas facilmente apenas dizendo como eles se transformam em relação ao grupo de gauge do MP, veja a tabela (2.1). Nesta tabela vemos que os férmions possuem três famílias de partículas, onde cada família se acopla de modo idêntico aos bósons de gauge. Além disso, temos que apenas os quarks interagem fortemente, interação inexistente entre os léptons, donde temos que estes são respectivamente tripletos e singletos de cor. Temos ainda que esses férmions são quirais $\psi_{L, R}=\frac{1}{2}\left(1 \mp \gamma^{5}\right)$, onde os férmions de mão esquerda (Left-handed) são dubletos de $S U(2)_{L}$, enquanto os de mão direita (Right-handed) são singletos. Por

\footnotetext{
${ }^{1} \mathrm{O}$ índice " $L$ " indica que só fermions de mão esquerda trasformam por $S U(2)_{L}$.
} 
fim, visando incorporar o eletromagnetismo atribui-se a hipercarga números quânticos que satisfaçam a relação de Gell-mann-Nishijima (2.7)

$$
Q=I_{3}+Y
$$

onde $Q$ será identificado como o gerador do $U(1)_{E M}$, grupo que resultará da quebra espontânea da simetria $S U(2)_{L} \otimes U(1)_{Y} \rightarrow U(1)_{E M}$, conforme veremos na seção (2.2.2).

\begin{tabular}{|c|c|c|c|c|c|}
\hline $1^{\circ}$ Família & $2^{\circ}$ Família & $3^{\circ}$ Família & $S U(3)_{c}$ & $S U(2)_{L}$ & $U(1)_{Y}$ \\
\hline$\left(\begin{array}{c}u_{L} \\
d_{L}\end{array}\right)$ & $\left(\begin{array}{c}c_{L} \\
s_{L}\end{array}\right)$ & $\left(\begin{array}{c}t_{L} \\
b_{L}\end{array}\right)$ & 3 & 2 & $\frac{1}{6}$ \\
$u_{R}$ & $c_{R}$ & $t_{R}$ & 3 & 1 & $\frac{2}{3}$ \\
$d_{R}$ & $s_{R}$ & $b_{R}$ & 3 & 1 & $-\frac{1}{3}$ \\
$\left(\begin{array}{c}\nu_{e L} \\
e_{L}\end{array}\right)$ & $\left(\begin{array}{c}\nu_{\mu L} \\
\mu_{L}\end{array}\right)$ & $\left(\begin{array}{c}\nu_{\tau L} \\
\tau_{L}\end{array}\right)$ & 1 & 2 & $-\frac{1}{2}$ \\
$\mu_{R}$ & $\tau_{R}$ & 1 & 1 & -1 \\
\hline
\end{tabular}

Tabela 2.1: Campos de matéria do Modelo Padrão com seus respectivos números quânticos.

No que segue, por simplicidade, vamos nos concentrar apenas em uma geração de quarks conforme [2]:

$$
\psi_{1}=\left(\begin{array}{l}
u \\
d
\end{array}\right)_{L}, \quad \psi_{2}=u_{R}, \quad \psi_{3}=d_{R}
$$

O que segue também é valido para o setor leptônico desde que façamos a seguinte correspondência ${ }^{2}$ :

$$
\psi_{1}=\left(\begin{array}{c}
\nu_{e} \\
e
\end{array}\right)_{L}, \quad \psi_{2}=0, \quad \psi_{3}=e_{R}
$$

Dado o conteúdo de matéria ${ }^{3}$ e o grupo de simetria do MP agora vamos montar a lagrangeana do modelo. Para tal a regra é inserir todos os termos possíveis que satisfaçam a simetria de gauge e sejam renormalizáveis. Para facilitar a exposição vamos dividir a lagrangiana do $\mathrm{MP} \mathcal{L}_{M P}$ em:

\footnotetext{
${ }^{2}$ No caso leptônico definimos $\psi_{2}=0$, pois no modelo padrão não existe neutrino de mão direita conforme a tabela (2.1).

${ }^{3} \mathrm{Na}$ verdade só após a quebra espontânea de simetria nossos campos estarão completos, com a inclusão do Higgs.
} 


$$
\mathcal{L}_{M P}=\mathcal{L}_{\text {fermion }}+\mathcal{L}_{\text {gauge }}+\mathcal{L}_{\text {escalar }}+\mathcal{L}_{\text {yuk }}
$$

onde $\mathcal{L}_{\text {fermion }}$ é a lagrangiana da parte fermiônica, $\mathcal{L}_{\text {gauge }}$ a lagrangiana dos campos de gauge, $\mathcal{L}_{\text {escalar }}$ se refere a parte escalar e $\mathcal{L}_{\text {yuk }}$ remete as interações de Yukawa férmions-escalar.

Comecemos com a parte fermiônica:

$$
\mathcal{L}_{\text {fermion }}=\sum_{j=1}^{3} i \bar{\psi}_{j}(x) \gamma^{\mu} D_{\mu} \psi_{j}(x)
$$

possuindo as seguintes derivadas covariantes ${ }^{4}$

$$
\begin{aligned}
& D_{\mu} \psi_{1}(x) \equiv\left(\partial_{\mu}+i g_{s} \frac{\lambda_{a}}{2} G_{\mu}^{a}+i g \frac{\sigma_{a}}{2} W_{\mu}^{a}+i g^{\prime} Y_{1} B_{\mu}\right) \psi_{1}(x) \\
& D_{\mu} \psi_{2}(x) \equiv\left(\partial_{\mu}+i g_{s} \frac{\lambda_{a}}{2} G_{\mu}^{a}+i g^{\prime} Y_{2} B_{\mu}\right) \psi_{2}(x) \\
& D_{\mu} \psi_{3}(x) \equiv\left(\partial_{\mu}+i g_{s} \frac{\lambda_{a}}{2} G_{\mu}^{a}+i g^{\prime} Y_{3} B_{\mu}\right) \psi_{3}(x),
\end{aligned}
$$

construídas de tal modo a garantir que $D_{\mu} \psi(x)$ transforme-se analogamente a $\psi(x)$, assegurando a invariância de (2.11) sob as transformações de gauge abaixo ${ }^{5}$ :

$$
\begin{aligned}
& \psi_{1}^{\eta}(x) \rightarrow \psi_{1}^{\prime} \eta(x)=e^{i Y_{1} \beta(x)} U_{L} U_{c \delta}^{\eta} \psi_{1}^{\delta}(x) \\
& \psi_{2}(x) \rightarrow \psi_{2}^{\prime}(x)=e^{i Y_{2} \beta(x)} \psi_{2}(x) \\
& \psi_{3}(x) \rightarrow \psi_{3}^{\prime}(x)=e^{i Y_{3} \beta(x)} \psi_{3}(x)
\end{aligned}
$$

com a transformação por $S U(2)_{L}$

$$
U_{L} \equiv \exp \left(i \frac{\sigma^{a}}{2} \alpha_{a}(x)\right)
$$

e a por $S U(3)_{c}$

$$
U_{c} \equiv \exp \left(i \frac{\lambda^{a}}{2} \theta_{a}(x)\right) .
$$

\footnotetext{
${ }^{4}$ Para $\psi_{i}$ sendo léptons a parte de interação com os gluons $\left(i g_{s} \frac{\lambda_{a}}{2} G_{\mu}^{a}\right)$ é nula, dado que esses férmions não possuem cor.

${ }^{5}$ Note que explicitamos os índices de cor, assim $\psi_{1}^{\eta}(x)$ é um quark com índice de cor $\eta=1,2,3$.
} 
A exigência da invariância da lagrangiana sob essas transformações impõe que os campos de gauge $B_{\mu}, W_{\mu}^{a}, G_{\mu}^{a}$ transformem-se infinitesimalmente segundo

$$
\begin{aligned}
& B_{\mu}(x) \rightarrow B_{\mu}^{\prime}(x)=B_{\mu}(x)-\frac{1}{g^{\prime}} \partial_{\mu} \beta(x) \\
& W_{\mu}^{a}(x) \rightarrow W_{\mu}^{\prime a}(x)=W_{\mu}^{a}(x)-\varepsilon^{j k a} \alpha^{j} W_{\mu}^{k}(x)-\frac{1}{g} \partial_{\mu} \alpha^{a}(x) \\
& G_{\mu}^{a}(x) \rightarrow G_{\mu}^{\prime a}(x)=G_{\mu}^{a}(x)-\varepsilon^{j k a} \theta^{j} G_{\mu}^{k}(x)-\frac{1}{g} \partial_{\mu} \theta^{a}(x) .
\end{aligned}
$$

Por fim, tendo em vista que os tensores de intensidade transformam-se do seguinte modo

$$
\begin{aligned}
B_{\mu \nu} & \rightarrow B_{\mu \nu} \\
\frac{\sigma^{a}}{2} W_{\mu \nu}^{a} & \rightarrow U_{L}\left(\frac{\sigma^{a}}{2} W_{\mu \nu}^{a}\right) U_{L}^{\dagger} \\
\frac{\lambda^{a}}{2} G_{\mu \nu}^{a} & \rightarrow U_{c}\left(\frac{\lambda^{a}}{2} G_{\mu \nu}^{a}\right) U_{c}^{\dagger},
\end{aligned}
$$

então ao construir a lagrangiana para os termos cinéticos dos campos de gauge como:

$$
\begin{aligned}
\mathcal{L}_{\text {gauge }} & =-\frac{1}{4} B^{\mu \nu} B_{\mu \nu}-\frac{1}{2} \operatorname{tr}\left(\frac{\sigma^{a}}{2} W_{\mu \nu}^{a} \frac{\sigma^{b}}{2} W_{\mu \nu}^{b}\right)-\frac{1}{2} \operatorname{tr}\left(\frac{\lambda^{a}}{2} G_{\mu \nu}^{a} \frac{\lambda^{b}}{2} G_{\mu \nu}^{b}\right) \\
& =-\frac{1}{4} B^{\mu \nu} B_{\mu \nu}-\frac{1}{4} W_{\mu \nu}^{a} W_{a}^{\mu \nu}-\frac{1}{4} G_{\mu \nu}^{a} G_{a}^{\mu \nu},
\end{aligned}
$$

temos de imediato a garantia da invariância de gauge para (2.18).

Note que apesar de sabermos experimentalmente da existência de bósons de gauge eletrofracos e férmions massivos não adicionamos de modo direto esses termos de massa na lagrangiana, pois:

- Termos de massa para os bósons de gauge $\left(\frac{M_{V}^{2}}{2} V_{\mu} V^{\mu}\right)$ violam a simetria de gauge, não sendo invariantes por (2.16).

- Termos de massa para os férmions $(-m \bar{\psi} \psi)$ também quebram a simetria explicitamente, pois esses termos ligam a parte Left-handed $\psi_{L}$ com a parte Righthanded $\psi_{R}$, as quais transformam-se diferentemente por $S U(2)_{L}$. 


$$
-m \bar{\psi} \psi=-m \bar{\psi}\left[\left(\frac{1-\gamma^{5}}{2}\right)+\left(\frac{1+\gamma^{5}}{2}\right)\right]^{2} \psi=-m\left(\bar{\psi}_{R} \psi_{L}+\bar{\psi}_{L} \psi_{R}\right)
$$

Na seção sobre Quebra Espontânea de Simetria, que está por vir, veremos como superar esses problemas.

\subsubsection{Interações Fortes (QCD)}

As lagrangeanas $\mathcal{L}_{\text {fermion }}(2.11)$ e $\mathcal{L}_{\text {gauge }}$ (2.18) possuem termos de interação forte, ou seja, termos de interação involvendo glúons os quais destacamos abaixo:

$$
\begin{aligned}
\mathcal{L}_{Q C D}^{i n t} & =-g_{s} G_{\mu}^{a} \sum_{f} \bar{q}_{f}^{\alpha} \gamma^{\mu}\left(\frac{\lambda^{a}}{2}\right)_{\alpha \delta} q_{f}^{\delta} \\
& +\frac{g_{s}}{2} f^{a b c}\left(\partial_{\mu} G_{\nu}^{a}-\partial_{\nu} G_{\mu}^{a}\right) G_{b}^{\mu} G_{c}^{\nu}-\frac{g_{s}^{2}}{4} f^{a b c} f_{a d e} G_{b}^{\mu} G_{c}^{\nu} G_{\mu}^{d} G_{\nu}^{e}
\end{aligned}
$$

onde $q_{f}^{\alpha}$ denota um quark de cor $\alpha$ e sabor $\mathrm{f}(\mathrm{u}, \mathrm{d}, \mathrm{c}, \mathrm{s}, \mathrm{t}, \mathrm{b})$. Na primeira linha de (2.20) temos as interações de cor entre quarks e gluons, e pelo carácter não abeliano do grupo $S U(3)_{c}$, temos também auto-interações cúbicas e quárticas entre glúons na segunda linha.

Vale destacar que a intensidade das interações fortes é governada por uma única constante de acoplamento, $g_{s}$, sendo independente das partículas que participam da interação. Fato que é consequência direta da simetria de gauge adotada, $S U(3)_{c}$.

\subsubsection{Interações com Correntes Carregadas}

Destacando os termos de interação entre bósons de gauge de $S U(2)_{L} \otimes U(1)_{Y}$ e férmions, presentes em $\mathcal{L}_{\text {fermion }}(2.11)$,

$$
-g \bar{\psi}_{1} \gamma^{\mu} \frac{1}{2}\left(\begin{array}{cc}
W_{\mu}^{3} & \sqrt{2} W_{\mu}^{+} \\
\sqrt{2} W_{\mu}^{-} & -W_{\mu}^{3}
\end{array}\right) \psi_{1}-g^{\prime} B_{\mu} \sum_{i=1}^{3} y_{i} \bar{\psi}_{i} \gamma^{\mu} \psi_{i}
$$

onde definimos os bósons de gauge carregados $W_{\mu}^{ \pm}$como

$$
W_{\mu}^{ \pm}=\frac{1}{\sqrt{2}}\left(W_{\mu}^{1} \mp i W_{\mu}^{2}\right) .
$$

Então, considerando apenas a primeira família de quarks e léptons, temos os 
seguintes termos de interação entre férmions e os bósons de gauge carregados, denominados de interações de correntes carregadas:

$$
\mathcal{L}_{c c}=-\frac{g}{\sqrt{2}}\left[W_{\mu}^{+}\left(\bar{u}_{L} \gamma^{\mu} d_{L}+\bar{\nu}_{e L} \gamma^{\mu} e_{L}\right)+W_{\mu}^{-}\left(\bar{d}_{L} \gamma^{\mu} u_{L}+\bar{e}_{L} \gamma^{\mu} \nu_{e L}\right)\right]
$$

\subsubsection{Interações com Correntes Neutras}

Observamos em (2.21) que ainda falta exibir as interações dos bósons de gauge eletrofracos $W_{\mu}^{3}$ e $B_{\mu}$. Assim como no caso das correntes carregadas vamos expressar essas interações em termos dos bósons de gauge que são auto-estados de massa, $Z_{\mu}$ e $A_{\mu}$, como veremos após a exposição do mecanismo de Higgs:

$$
\left(\begin{array}{c}
W_{\mu}^{3} \\
B_{\mu}
\end{array}\right) \equiv\left(\begin{array}{cc}
\cos \theta_{W} & \sin \theta_{W} \\
-\sin \theta_{W} & \cos \theta_{W}
\end{array}\right)\left(\begin{array}{c}
Z_{\mu} \\
A_{\mu}
\end{array}\right)
$$

Onde o ângulo $\theta_{W}$, denominado ângulo de mistura eletrofraco, é dado por

$$
\tan \theta_{W} \equiv \frac{g^{\prime}}{g}
$$

que garante a interpretação do $A_{\mu}$ como fóton, pois providencia dentre outras coisas que este campo não se acople com os neutrinos, que possuem carga elétrica nula. Então, usando (2.24) vemos que a lagrangiana das correntes neutras é diagonal nos sabores possuindo a seguinte forma

$$
\mathcal{L}_{N C}=-\sum_{i=1}^{3} \bar{\psi}_{i} \gamma^{\mu}\left[A_{\mu}\left(g I_{3} \sin \theta_{W}+g^{\prime} y_{i} \cos \theta_{W}\right)+Z_{\mu}\left(g I_{3} \cos \theta_{W}-g^{\prime} y_{i} \sin \theta_{W}\right)\right] \psi_{i}
$$

Essa expressão pode ser simplificada se tomarmos partido da relação de GellMan-Nishijimma (2.7) e da expressão (2.25) que pode ser reescrita como:

$$
\begin{gathered}
g \sin \theta_{W}=g^{\prime} \cos \theta_{W} \equiv e \\
g \cos \theta_{W}+g^{\prime} \sin \theta_{W}=\sqrt{g^{2}+g^{\prime 2}}=\frac{e}{\sin \theta_{W} \cos \theta_{W}} .
\end{gathered}
$$

Assim, 


$$
\mathcal{L}_{N C}=-\sum_{i=1}^{3} \bar{\psi}_{i} \gamma^{\mu}\left[e Q_{i} A_{\mu}+\frac{e}{\sin \theta_{W} \cos \theta_{W}}\left(I_{3}-\sin ^{2} \theta_{W} Q_{i}\right) Z_{\mu}\right] \psi_{i}
$$

Podemos ainda introduzir uma notação mais usual:

$$
\mathcal{L}_{N C}=-\sum_{f} \bar{f} \gamma^{\mu}\left[e Q_{i} A_{\mu}+\frac{e}{\sin \theta_{W} \cos \theta_{W}}\left(g_{V}^{f}-g_{A}^{f} \gamma^{5}\right) Z_{\mu}\right] f
$$

$\operatorname{com} g_{V}^{f}=I_{3}^{f}$ e $g_{A}^{f}=I_{3}^{f}\left(1-4\left|Q_{f}\right| \sin ^{2} \theta_{W}\right)$ explicitados na tabela (2.2) para os diferentes férmions $f$.

\begin{tabular}{|c|c|c|}
\hline Férmions & $g_{V}^{f}$ & $g_{A}^{f}$ \\
\hline \hline quarks up & $\frac{1}{2}-\frac{4}{3} \sin ^{2} \theta_{W}$ & $\frac{1}{2}$ \\
\hline quarks down & $-\frac{1}{2}+\frac{2}{3} \sin ^{2} \theta_{W}$ & $-\frac{1}{2}$ \\
\hline neutrinos & $\frac{1}{2}$ & $\frac{1}{2}$ \\
\hline léptons carregados & $-\frac{1}{2}+2 \sin ^{2} \theta_{W}$ & $-\frac{1}{2}$ \\
\hline
\end{tabular}

Tabela 2.2: Acoplamentos vetorial e axial dos quarks e léptons com o $Z^{0}$.

Observamos que as interações fóton-férmions, presentes no primeiro termo de (2.30), possuem um peso que é proporcinal à carga elétrica $Q$. Garantindo como já destacado que os neutrinos não se acoplem com os fótons. Por fim, se compararmos esse termo com a QED interpretamos que " $e$ ", definido em (2.27), faz o papel de constante de acoplamento eletromagnética sendo simplismente o valor absoluto da carga do elétron.

\subsubsection{Interação entre os bósons de gauge eletrofracos}

Os bósons vetoriais eletrofracos possuem termos de interação cúbicos e quárticos, todos provenientes da parte associada a $S U(2)_{L}$ em $\mathcal{L}_{\text {gauge }}(2.18)$.

$$
\mathcal{L}=-\frac{1}{4} W_{\mu \nu}^{a} W_{a}^{\mu \nu}
$$

Os termos cúbicos são 


$$
\begin{aligned}
\mathcal{L}_{3} & =-\frac{1}{2} g \varepsilon^{a b c} W_{a}^{\mu \nu} W_{b \mu} W_{c \nu} \\
& =\mathcal{L}_{W W \gamma}+\mathcal{L}_{W W Z}
\end{aligned}
$$

com

$$
\begin{aligned}
\mathcal{L}_{W W \gamma}= & i e\left[\left(\partial^{\mu} W^{-\nu}-\partial^{\nu} W^{-\mu}\right) W_{\mu}^{+} A_{\nu}-\left(\partial^{\mu} W^{+\nu}-\partial^{\nu} W^{+\mu}\right) W_{\mu}^{-} A_{\nu}\right. \\
& \left.+W_{\mu}^{-} W_{\nu}^{+}\left(\partial^{\mu} A^{\nu}-\partial^{\nu} A^{\mu}\right)\right] \\
\mathcal{L}_{W W Z}= & i e \cot \theta_{W}\left[\left(\partial^{\mu} W^{-\nu}-\partial^{\nu} W^{-\mu}\right) W_{\mu}^{+} Z_{\nu}-\left(\partial^{\mu} W^{+\nu}-\partial^{\nu} W^{+\mu}\right) W_{\mu}^{-} Z_{\nu}\right. \\
& \left.+W_{\mu}^{-} W_{\nu}^{+}\left(\partial^{\mu} Z^{\nu}-\partial^{\nu} Z^{\mu}\right)\right]
\end{aligned}
$$

Já os termos quárticos

$$
\begin{aligned}
\mathcal{L}_{4} & =-\frac{1}{4} g^{2} \varepsilon_{a b c} \varepsilon_{a d e} W_{\mu}^{b} W_{\nu}^{c} W^{d \mu} W^{e \nu} \\
& =-\frac{1}{4} g^{2}\left[\left(W_{\mu}^{a} W_{a}^{\mu}\right)^{2}-W_{a \mu} W_{\nu}^{a} W_{b}^{\mu} W^{b \nu}\right] \\
& =\mathcal{L}_{W W W W}+\mathcal{L}_{W W Z Z}+\mathcal{L}_{W W \gamma \gamma}+\mathcal{L}_{W W Z \gamma}
\end{aligned}
$$

com

$$
\begin{aligned}
\mathcal{L}_{W W W W} & =-\frac{e^{2}}{2 \sin ^{2} \theta_{W}}\left[\left(W_{\mu}^{+} W^{-\mu}\right)^{2}-W_{\mu}^{+} W^{+\mu} W_{\nu}^{-} W^{-\nu}\right] \\
\mathcal{L}_{W W Z Z} & =-e^{2} \cot ^{2} \theta_{W}\left[W_{\mu}^{+} W^{-\mu} Z_{\nu} Z^{\nu}-W_{\mu}^{+} Z^{\mu} W_{\nu}^{-} Z^{\nu}\right] \\
\mathcal{L}_{W W \gamma \gamma} & =-e^{2}\left[W_{\mu}^{+} W^{-\mu} A_{\nu} A^{\nu}-W_{\mu}^{+} A^{\mu} W_{\nu}^{-} A^{\nu}\right] \\
\mathcal{L}_{W W Z \gamma} & =-e^{2} \cot \theta_{W}\left[2 W_{\mu}^{+} W^{-\mu} Z_{\nu} A^{\nu}-W_{\mu}^{+} Z^{\mu} W_{\nu}^{-} A^{\nu}-W_{\mu}^{+} A^{\mu} W_{\nu}^{-} Z^{\nu}\right]
\end{aligned}
$$

Vale destacar que não vemos vértices com apenas fótons e Z's devido a álgebra do grupo $S U(2)_{L}$ não permitir, forçando sempre a presença de pelo menos dois bósons carregados $\mathrm{W}$. 


\subsection{Quebra Espontânea de Simetria}

Apesar do fóton ser um bóson não massivo os bósons de gauge $W^{ \pm}$e $Z$ possuem massas detectadas experimentalmente de alto valor: $m_{W} \approx 80 \mathrm{GeV}$ e $m_{Z} \approx 90$ $\mathrm{GeV}$, donde temos a necessidade iminente de inserir um mecanismo que gere massa a esses bósons sem quebrar a simetria de gauge explicitamente. Nesta seção analisaremos o mecanismo utilizado pelo MP para providenciar essas massas.

\subsubsection{O Teorema de Goldstone}

Considere a lagrangiana formada pelo campo escalar complexo $\phi=\frac{1}{\sqrt{2}}\left(\phi_{1}+i \phi_{2}\right)$

$$
\mathcal{L}=\left(\partial_{\nu} \phi\right)^{\dagger} \partial^{\nu} \phi-V(\phi), \quad V(\phi)=\mu^{2} \phi^{\dagger} \phi+\lambda\left(\phi^{\dagger} \phi\right)^{2}, \quad(\lambda>0)
$$

que é invariante sob transformações de fase globais do grupo de simetria U(1), $\phi \rightarrow e^{i \alpha} \phi$. Para que o potencial seja limitado inferiormente tomamos $\lambda>0$, já no que concerne o comportamento de $V(\phi)$ quanto ao sinal de $\mu^{2}$ vamos reescrever a lagrangiana em termos de $\phi_{1}$ e $\phi_{2}$ para procedermos a análise:

$$
\mathcal{L}=\frac{1}{2}\left(\partial_{\nu} \phi_{1} \partial^{\nu} \phi_{1}+\partial_{\nu} \phi_{2} \partial^{\nu} \phi_{2}\right)-\frac{\mu^{2}}{2}\left(\phi_{1}^{2}+\phi_{2}^{2}\right)-\frac{1}{4} \lambda\left(\phi_{1}^{2}+\phi_{2}^{2}\right)^{2}, \quad(\lambda>0)
$$

a) $\mu^{2}>0$ : Temos que o mínimo do potencial está em $\phi=0$ e o vácuo, assim como a lagrangiana, é simétrico por transformações $U(1)$ globais. Neste caso interpretamos (2.41) como uma lagrangeana de dois campos escalares reais de mesma massa $\mu$ com um termo de interação proporcional a $\lambda$.

b) $\mu^{2}<0$ : Temos o mínimo do potencial em um círculo de raio $v=\sqrt{-\frac{\mu^{2}}{\lambda}}$ no plano $\left(\phi_{1}, \phi_{2}\right)$. Neste caso apesar da lagrangeana ser invariante por U(1) o mesmo não acontece com o vácuo por este ser infinitamente degenerado. Para proceder a análise deste caso vamos, sem perda de generalidade, escolher como vácuo o ponto $\phi_{1}=v, \phi_{2}=0$ e expandir $\mathcal{L}$ em função dos campos $\eta$ e $\xi$ entorno desse como segue:

$$
\phi(x)=\frac{1}{\sqrt{2}}(v+\eta(x)+i \xi(x))
$$




$$
\mathcal{L}=\frac{1}{2}\left(\partial_{\nu} \eta \partial^{\nu} \eta+\partial_{\nu} \xi \partial^{\nu} \xi\right)+\mu^{2} \eta^{2}-\lambda v \eta\left(\eta^{2}+\xi^{2}\right)-\frac{\lambda}{4}\left(\eta^{2}+\xi^{2}\right)^{2}-V(v)
$$

De (2.43) interpretamos que no caso $\mu^{2}<0$ temos dois campos escalares reais no espectro, um massivo $(\eta)$ de massa $m_{\eta}=\sqrt{-2 \mu^{2}}$ e outro não massivo $(\xi)$, que é denominado de bóson de Goldstone. O fato deste campo ser não massivo tem origem na forma do potencial, veja figura (2.1), o qual é plano em direções tangentes a $(\xi)$ não oferecendo resistência a excitações nessa direção.

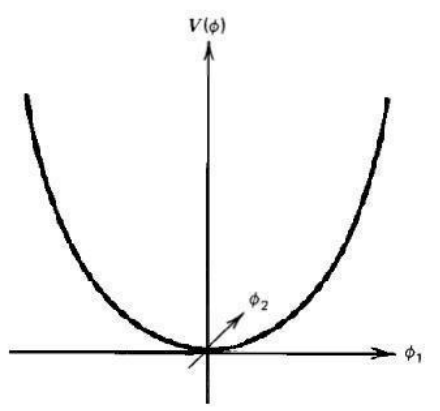

(a)

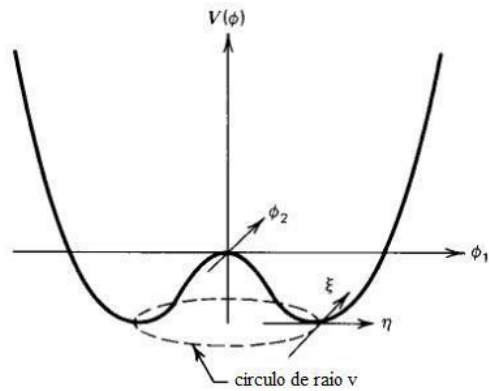

(b)

Figura 2.1: Potencial da lagrangeana (2.41) no caso (a) com $\mu^{2}>0$ e no caso (b) com $\mu^{2}<0$, onde temos a quebra espontânea da simetria.

Este é apenas um caso particular do Teorema de Goldstone: "Se uma lagrangeana é invariante sob um grupo de simetria global e continuo $G$, mas se o vácuo for invariante sob um subgrupo $H \subset G$, então teremos para cada gerador quebrado, i.e., geradores de $G$ que não pertencem a $H$, uma partícula de spin-0 sem massa (bóson de Goldstone)".

\subsubsection{O Mecanismo de Higgs}

Nesta subseção veremos como os bósons de Goldstone cooperando com os bósons de gauge podem gerar massas para esses últimos. Esta é a base do Mecanismo de Higgs do Modelo Padrão, que é o modo mais simples de superar o problema de gerar massas para os bósons de gauge $\left(W^{ \pm}, Z\right)$, termos que não podem ser introduzidos de modo direto na lagrangeana do MP por violar explicitamente a simetria do modelo.

Esse mecanismo consiste na inserção do dubleto escalar complexo 


$$
\phi \equiv\left(\begin{array}{c}
\phi^{+} \\
\phi^{0}
\end{array}\right)
$$

que transforma-se como um singleto por $S U(3)_{c}$, um dubleto por $S U(2)_{L}$ e possui hipercarga $Y_{\phi}=Q_{\phi}-I_{3}=\frac{1}{2}$. A esse dubleto associamos a lagrangiana (2.45) análoga a lagrangiana de Goldstone, só que agora invariante por $S U(3)_{c} \otimes S U(2)_{L} \otimes$ $U(1)_{Y}$ local.

$$
\begin{aligned}
\mathcal{L}_{\text {escalar }} & =\left(D_{\nu} \phi\right)^{\dagger} D^{\nu} \phi-\mu^{2} \phi^{\dagger} \phi-\lambda\left(\phi^{\dagger} \phi\right)^{2}, \quad\left(\lambda>0 ; \mu^{2}<0\right) \\
D_{\mu} \phi & =\left(\partial_{\mu}+i g I^{a} W_{\mu}^{a}+i g^{\prime} Y_{\phi} B_{\mu}\right) \phi
\end{aligned}
$$

Como $\mu^{2}<0$ aqui também teremos um vácuo degenerado que é dado pelo mínimo do potencial de $(2.45)$

$$
\left|\phi^{+}\right|^{2}+\left|\phi^{0}\right|^{2}=-\frac{\mu^{2}}{2 \lambda} .
$$

Visando manter a carga elétrica conservada escolhe-se um vácuo de tal modo que apenas o campo escalar neutro $\left(\phi^{0}\right)$ adquire um valor esperado no vácuo (vev).

$$
<0|\phi| 0>=\left(\begin{array}{c}
0 \\
v / \sqrt{2}
\end{array}\right), \quad v \equiv \sqrt{-\frac{\mu^{2}}{\lambda}}
$$

Vamos explicitar como a escolha desse vácuo quebra a simetria do modo desejado, a saber, $S U(2)_{L} \otimes U(1)_{Y} \rightarrow U(1)_{E M}$ conforme [1].

Para o vácuo ser invariante sobre um gerador $\tau$ temos que ter satisfeita $\exp (i \alpha \tau)<$ $\phi>_{0}=<\phi>_{0}$, i.e., $\tau<\phi>_{0}=0$. Deste modo facilmente constatamos que todos os 4 geradores do grupo $S U(2)_{L} \otimes U(1)_{Y}$ são quebrados

$$
\begin{aligned}
\sigma^{1}<\phi>_{0} & =\left(\begin{array}{c}
v / \sqrt{2} \\
0
\end{array}\right) \neq 0 \\
\sigma^{2}<\phi>_{0} & =\left(\begin{array}{c}
-i v / \sqrt{2} \\
0
\end{array}\right) \neq 0 \\
\sigma^{3}<\phi>_{0} & =\left(\begin{array}{c}
0 \\
-v / \sqrt{2}
\end{array}\right) \neq 0 \\
Y<\phi>_{0} & =1<\phi>_{0} \neq 0
\end{aligned}
$$


contudo o gerador do grupo $U(1)_{E M}$ continua deixando o vácuo invariante

$$
Q<\phi>_{0}=\left(I_{3}+Y\right)<\phi>_{0}=0
$$

então pelo teorema de Goldstone teremos 3 bósons não massivos.

Parametrizando a lagrangiana em relação ao mínimo do potencial escrevendo

$$
\phi(x)=\exp \left(\frac{i \zeta^{a}(x) \sigma^{a}}{2 v}\right)\left(\begin{array}{c}
0 \\
\frac{v+H(x)}{\sqrt{2}}
\end{array}\right)
$$

com quatro campos reais $\zeta^{a}(x)$ e $H(x)$. Note que apesar do terceiro gerador quebrado ortogonal a $Q$ ser $K=\frac{\sigma^{3}}{2}-Y$, utilizamos na expansão $\sigma^{3}$, pois o resultado é o mesmo tendo em vista (2.50) e que $\sigma^{3}=K+Q$.

Lembrando que os bósons de Goldstone nos permitem distinguir os diferentes vácuos sem custo de energia, então identificamos os 3 campos $\zeta^{a}(x)$ com os bósons associados aos 3 geradores quebrados. Mas como veremos podemos fazer estes campos sumirem do espectro com uma simples escolha de gauge, denominado gauge unitário:

$$
\phi \rightarrow \phi^{\prime}=\exp \left(-\frac{i \zeta^{a} \sigma^{a}}{2 v}\right) \phi=\left(\begin{array}{c}
0 \\
\frac{v+H(x)}{\sqrt{2}}
\end{array}\right)
$$

Escrevendo $\mathcal{L}_{\text {escalar }}(2.45)$ no gauge unitário

$$
\begin{aligned}
\mathcal{L}_{\text {escalar }} & =\frac{1}{2}\left(\partial_{\mu} H\right)^{2}-\lambda v^{2} H^{2}-\frac{1}{2} \lambda v^{2} H^{2}-\lambda v H^{3}-\frac{\lambda}{4} H^{4} \\
& +\frac{1}{4} g^{2}(v+H)^{2} W_{\mu}^{+} W^{-\mu}+\frac{1}{8} \sqrt{g^{2}+g^{\prime 2}}(v+H)^{2} Z_{\mu} Z^{\mu}
\end{aligned}
$$

vemos que os bósons vetoriais $W^{ \pm}$e $Z$ ganharam massa

$$
M_{W}=\frac{1}{2} g v ; \quad M_{Z}=\frac{1}{2} v \sqrt{g^{2}+g^{\prime 2}} ; \quad M_{A}=0,
$$

isso é devido a absorção dos bósons de Goldstone como suas componentes longitudinais, enquanto isso o fóton continua não massivo como desejado, pois o gerador $Q$ do $U(1)_{E M}$ não foi quebrado.

Note que além de gerar massas para os bósons de gauge em (2.53), também obtemos com este procedimento um campo escalar real $H(x)$, nomeado de Higgs. Nas seções posteriores detalharemos as interações e os limites teóricos e experi- 
mentais sobre a massa desta que é a única partícula do MP não observada experimentalmente até a presente data.

\subsubsection{Massa para os férmions e a matriz CKM}

Para solucionar o problema de gerar massa aos férmions, o MP toma partido de uma possibilidade aberta através da introdução do campo escalar complexo (2.44) no modelo. A possibilidade que estamos nos referindo diz respeito a construção de interações de Yukawa, as quais são renormalizáveis e satisfazem a simetria de gauge.

Para expressarmos essa parte da lagrangeana do MP vamos explicitar todas as famílias, pois nesse caso existirá a possibilidade de mistura entre elas mesmo antes de passarmos da base de gauge (base que utilizamos até o momento) à base de massas, cujos campos são auto-estados da matriz de massa.

$$
\begin{aligned}
& \mathcal{L}_{y u k}=-\left(\begin{array}{cc}
\bar{u}_{i}^{\prime}, & \bar{d}_{i}^{\prime}
\end{array}\right)_{L}\left[h_{(d)}^{i j}\left(\begin{array}{c}
\phi^{+} \\
\phi^{0}
\end{array}\right) d_{j R}^{\prime}+h_{(u)}^{i j}\left(\begin{array}{c}
\phi^{0 *} \\
-\phi^{-}
\end{array}\right) u_{j R}^{\prime}\right] \\
& -\left(\begin{array}{cc}
\overline{\nu_{i}^{\prime}}, & \bar{l}_{i}^{\prime}
\end{array}\right)_{L} h_{(l)}^{i j}\left(\begin{array}{c}
\phi^{+} \\
\phi^{0}
\end{array}\right) l_{j R}^{\prime}+\text { h.c. }
\end{aligned}
$$

Com $h_{(u)}^{i j}, h_{(d)}^{i j}, h_{(l)}^{i j}(\mathrm{i}, \mathrm{j}=1,2,3)$ arbitrários, ou seja, não são preditos pelo MP tendo que ser obtidos experimentalmente. A "linha" indica que estamos na base de gauge com a seguinte notação:

$$
\begin{aligned}
\nu_{i}^{\prime} & =\nu_{e}^{\prime}, \nu_{\mu}^{\prime}, \nu_{\tau}^{\prime} \\
l_{i}^{\prime} & =e^{\prime}, \mu^{\prime}, \tau^{\prime} \\
u_{i}^{\prime} & =u^{\prime}, c^{\prime}, t^{\prime} \\
d_{i}^{\prime} & =d^{\prime}, s^{\prime}, b^{\prime}
\end{aligned}
$$

Além disso note que utilizamos além do dubleto escalar complexo usual (2.44), fizemos uso também no segundo termo do dubleto

$$
\phi^{c} \equiv i \sigma^{2} \phi^{*}=\left(\begin{array}{c}
\phi^{0 *} \\
-\phi^{-}
\end{array}\right)
$$


que será responsável por dar massas aos quarks up depois da quebra da simetria, veja:

$$
\mathcal{L}_{y u k}=-\left(1+\frac{H}{v}\right)\left(\bar{d}_{i L}^{\prime} \frac{v}{\sqrt{2}} h_{v(d)}^{i j} d_{j R}^{\prime}+\bar{u}_{i L}^{\prime} \frac{v}{\sqrt{2}} h_{(u)}^{i j} u_{j R}^{\prime}+\bar{l}_{i L}^{\prime} \frac{v}{\sqrt{2}} h_{(l)}^{i j} l_{j R}^{\prime}+\text { h.c. }\right)
$$

As matrizes

$$
M_{i j}^{(d)}=\frac{v}{\sqrt{2}} h_{i j}^{(d)} ; \quad M_{i j}^{(u)}=\frac{v}{\sqrt{2}} h_{i j}^{(u)} ; \quad M_{i j}^{(l)}=\frac{v}{\sqrt{2}} h_{i j}^{(l)}
$$

são denomindas matrizes de massa. Diagonalizando-as podemos expressar os campos fermiônicos de auto-estados de gauge para auto-estados de massa.

Tendo em vista que toda matriz quadrada pode ser diagonalizada por uma transformação biunitária, então podemos sempre encontrar as matrizes $U^{(u)} L, R$, $U_{L, R}^{(d)}, U^{(l)}{ }_{L, R}$ que diagonalizam (2.59):

$$
\begin{aligned}
& \mathcal{M}^{(\mathrm{u})}=U_{L}^{(u) \dagger} M^{(u)} U_{R}^{(u)}=\operatorname{diag}\left(m_{u}, m_{c}, m_{t}\right) \\
& \mathcal{M}^{(\mathrm{d})}=U_{L}^{(d) \dagger} M^{(d)} U_{R}^{(d)}=\operatorname{diag}\left(m_{d}, m_{s}, m_{b}\right) \\
& \mathcal{M}^{(\mathrm{l})}=U_{L}^{(l) \dagger} M^{(l)} U_{R}^{(l)}=\operatorname{diag}\left(m_{e}, m_{\mu}, m_{\tau}\right)
\end{aligned}
$$

Então,

$$
\mathcal{L}_{y u k}=-\left(1+\frac{H}{v}\right)\left(\bar{d}_{i L} \mathcal{M}_{\mathrm{ij}}^{(\mathrm{d})} d_{j R}+\bar{u}_{i L} \mathcal{M}_{\mathrm{ij}}^{(\mathrm{u})} u_{j R}+\bar{l}_{i L} \mathcal{M}_{\mathrm{ij}}^{(\mathrm{l})} l_{j R}+h . c .\right)
$$

os auto-estados de massa, denotados sem "linha", são obtidos dos auto-estados de gauge por:

$$
\begin{array}{rlrl}
u_{i L} & =U_{i j L}^{(u) \dagger} u_{j L}^{\prime} ; & d_{i L}=U_{i j L}^{(d) \dagger} d_{j L}^{\prime} ; & l_{i L}=U_{i j L}^{(l) \dagger} l_{j L}^{\prime} \\
u_{i R}=U_{i j R}^{(u) \dagger} u_{j R}^{\prime} ; & d_{i R}=U_{i j R}^{(d) \dagger} d_{j R}^{\prime} ; & l_{i R}=U_{i j R}^{(l) \dagger} l_{j R}^{\prime}
\end{array}
$$

Observe que o acoplamento dos férmions com o bóson de Higgs é diretamente proporcional a massa dos férmions. Donde temos que os neutrinos, por serem considerados não massivos no MP, não se acoplam com este bóson.

Agora que apresentamos a lagrangeana (2.63) em termos dos auto-estados de 
massa, o próximo passo é ver o que muda se expressarmos as outras partes da lagrangeana do MP em termos desses auto-estados. De imediato temos que os termos cinéticos e a lagrangiana das correntes neutras possuem a mesma forma da base de gauge por serem diagonais nos sabores:

$$
\begin{aligned}
& \bar{f}_{L}^{\prime} f_{L}^{\prime}=\bar{f}_{L} U_{L}^{(f)} U_{L}^{(f) \dagger} f_{L}=\bar{f}_{L} f_{L} \\
& \bar{f}_{R}^{\prime} f_{R}^{\prime}=\bar{f}_{R} U_{R}^{(f)} U_{R}^{(f) \dagger} f_{R}=\bar{f}_{R} f_{R}, \quad f=(u, d, l)
\end{aligned}
$$

Essa invariância de sabor no MP para as correntes neutras é conhecida como mecanismo de GIM.

Ao analisar a lagrangeana para correntes carregadas vamos explorar o fato dos neutrinos não terem termos de massa, o que possibilita definí-los na base de massas como:

$$
\nu_{i} \equiv U_{i j}^{(l) \dagger} \nu_{j}^{\prime}
$$

Assim podemos escrever a lagrangeana para correntes carregadas (2.23), neste caso explicitando todas as famílias, como:

$$
\mathcal{L}_{c c}=-\frac{g}{\sqrt{2}}\left[W_{\mu}^{+}\left(\bar{u}_{i L} \gamma^{\mu} V_{i j} d_{j L}+\bar{\nu}_{i L} \gamma^{\mu} l_{i L}\right)+W_{\mu}^{-}\left(\bar{d}_{i L} \gamma^{\mu} V_{i j}^{\dagger} u_{j L}+\bar{l}_{i L} \gamma^{\mu} \nu_{i L}\right)\right]
$$

Com V definido do seguinte modo

$$
V \equiv U_{L}^{(u) \dagger} U_{L}^{(d)}=\left(\begin{array}{ccc}
V_{u d} & V_{u s} & V_{u b} \\
V_{c d} & V_{c s} & V_{c b} \\
V_{t d} & V_{t s} & V_{t b}
\end{array}\right)
$$

Então vemos que o termo leptônico mantém a sua forma graças a definição (2.66), entretanto na parte hadrônica aparece uma matriz $V$, denominada matriz de Cabibbo-Kobayashi-Maskawa (CKM). Essa matriz contém informação sobre todas as possíveis transições de sabor de qualquer quark up para todos os quarks down. Estes processos de troca de sabores entre os quarks são obtidos experimentalmente possuindo as seguintes magnitudes para os elementos da matriz CKM [10]. 


$$
V=\left(\begin{array}{ccc}
0.974 & 0.226 & 0.004 \\
0.225 & 0.973 & 0.041 \\
0.009 & 0.041 & 1
\end{array}\right)
$$

\subsubsection{As interações do Higgs}

Visando gerar massa para os bósons de gauge e férmions no MP introduzimos um dubleto escalar complexo. Esse dubleto, após a quebra espontânea de simetria, tem 3 graus de liberdade (os bósons de Goldstone) absorvidos para gerar massa para os bósons vetoriais deixando apenas um campo escalar real no espectro, o Higgs. Essa partícula é a única prevista pelo MP que até a presente data não foi detectada experimentalmente.

Uma das questões fundamentais na busca por qualquer partícula é saber quais são seus termos de interação. No caso do Higgs do MP podemos obter esses termos da lagrangiana do campo escalar (2.53) e da lagrangiana de Yukawa (2.63). Destacando dessas os termos que possuem o Higgs

$$
\begin{aligned}
\mathcal{L}_{\text {Higgs }} & =\frac{1}{2}\left(\partial_{\mu} H \partial^{\mu} H-M_{H}^{2} H^{2}\right)-\frac{M_{H}^{2}}{2 v} H^{3}-\frac{M_{H}^{2}}{8 v^{2}} H^{4} \\
& +M_{W}^{2} W_{\mu}^{\dagger} W^{\mu}\left(\frac{2}{v} H+\frac{H^{2}}{v^{2}}\right)+\frac{M_{Z}^{2}}{2} Z_{\mu} Z^{\mu}\left(\frac{2}{v} H+\frac{H^{2}}{v^{2}}\right) \\
& -\frac{H}{v}\left(\bar{d}_{i L} \mathcal{M}_{\mathrm{ij}}^{(\mathrm{d})} d_{j R}+\bar{u}_{i L} \mathcal{M}_{\mathrm{ij}}^{(\mathrm{u})} u_{j R}+\bar{l}_{i L} \mathcal{M}_{\mathrm{ij}}^{(\mathrm{l})} l_{j R}+h . c .\right),
\end{aligned}
$$

com

$$
M_{H}=\sqrt{2 \mu^{2}}=\sqrt{2 \lambda} v .
$$

Dos termos de interação em $\mathcal{L}_{\text {Higgs }}$ observamos que uma característica peculiar desse campo escalar é possuir acoplamentos sempre dependentes da massa da partícula com quem ele interage e do vev $(v)$.

\subsection{Simetria Custodial}

Observando as massas dos bósons de gauge W e Z (2.54) e a definição do ângulo de mistura temos que o MP satisfaz em ordem de árvore: 


$$
\rho \equiv \frac{M_{W}^{2}}{M_{Z}^{2} \cos ^{2} \theta_{W}}=1 .
$$

Essa relação é apenas uma das facetas da existência da simetria $S U(2)$ custodial $[3,11,12,13]$, uma simetria aproximada do MP. Para melhor analisá-la vamos definir a matriz de Higgs conforme ref. [13]

$$
\Phi=\frac{1}{\sqrt{2}}\left(\phi^{c}, \phi\right)=\frac{1}{\sqrt{2}}\left(\begin{array}{cc}
\phi^{0 *} & \phi^{+} \\
-\phi^{-} & \phi^{0}
\end{array}\right),
$$

com a simetria de gauge $S U(2)_{L} \otimes U(1)_{Y}$ agindo sobre $\Phi$ do seguinte modo

$$
\begin{aligned}
& \Phi \rightarrow U_{L} \Phi, S U(2)_{L} \\
& \Phi \rightarrow \Phi e^{-i \sigma_{3} \beta}, U(1)_{Y} .
\end{aligned}
$$

Assim podemos expressar $\mathcal{L}_{\text {escalar }}(2.45)$ como

$$
\mathcal{L}_{\text {escalar }}=\operatorname{tr}\left(\left(D_{\nu} \Phi\right)^{\dagger} D^{\nu} \Phi\right)-V(\Phi)
$$

desde que o potencial e a derivada covariante sejam

$$
\begin{aligned}
V(\Phi) & =\mu^{2} \operatorname{tr}\left(\Phi^{\dagger} \Phi\right)+\lambda\left[\operatorname{tr}\left(\Phi^{\dagger} \Phi\right)\right]^{2} \\
D_{\mu} \Phi & =\partial_{\mu} \Phi+i g \frac{\sigma^{a}}{2} W_{\mu}^{a} \Phi-i \frac{g^{\prime}}{2} B_{\mu} \Phi \sigma_{3},
\end{aligned}
$$

onde a matriz $\sigma_{3}$ aparece na transformação (2.75) e na derivada covariante devido a $\phi$ e $\phi^{c}$ terem hipercargas com sinais opostos.

Considere agora o caso em que o acoplamento da hipercarga é nulo, $g^{\prime}=0$ $\left(\sin \theta_{W}=0\right)$. Neste contexto a simetria global $S U(2)_{R}$

$$
\Phi \rightarrow \Phi U_{R}^{\dagger}
$$

fica manifesta em $\mathcal{L}_{\text {escalar }}(2.76)$. Donde temos que antes da quebra espontânea de simetria $\mathcal{L}_{\text {escalar }}$, no limite $g^{\prime}=0\left(\sin \theta_{W}=0\right)$, satisfaz a simetria global $S U(2)_{L} \otimes$ $S U(2)_{R}$

$$
\Phi \rightarrow U_{L} \Phi U_{R}^{\dagger},
$$


onde $S U(2)_{L}$ global provém da simetria de gauge.

Tomando o valor esperado do vácuo para o Higgs

$$
<0|\Phi| 0>=\frac{v}{\sqrt{2}}\left(\begin{array}{ll}
1 & 0 \\
0 & 1
\end{array}\right)
$$

temos a quebra da simetria

$$
S U(2)_{L} \otimes S U(2)_{R} \rightarrow S U(2)_{L+R}
$$

pois vemos que (2.80) fica satisfeita apenas no caso $U_{L}=U_{R}$.

$$
<\Phi>\rightarrow U_{L}<\Phi>U_{L}^{\dagger}
$$

Essa simetria resultante $S U(2)_{L+R}$ intitulamos simetria custodial.

Sob essa simetria os 3 bósons de gauge $W_{\mu}^{1,2,3}$ trasformam-se como um tripleto, o que implica que eles possuem a mesma massa. Combinando esse resultado com

$$
\begin{aligned}
\frac{1}{2} M_{Z}^{2} Z_{\mu} Z^{\mu} & =\frac{1}{2} M_{Z}^{2}\left(\cos \theta_{W} W_{\mu}^{3}-\sin \theta_{W} B_{\mu}\right)^{2} \\
& =\frac{1}{2} M_{Z}^{2} \cos ^{2} \theta_{W} W_{\mu}^{3} W^{3 \mu}+\ldots
\end{aligned}
$$

Então a massa de $W^{3}$ pela simetria custodial $S U(2)_{L+R}$ é

$$
M_{W}^{2}=M_{Z}^{2} \cos ^{2} \theta_{W}
$$

que implica $\rho=1$. Todavia no MP temos que $g^{\prime} \neq 0$, implicando na quebra explícita da simetria custodial devido as interações do grupo de gauge $U(1)_{Y}$, além disso temos que as correções aos propagadores do $\mathrm{W}$ e $\mathrm{Z}$ oriundas de férmions massivos quebram essa simetria. Devido a esses termos aparecerão correções a 1-loop para $\rho=1$, que apesar de serem muito pequenas podem ser detectadas experimentalmente.

A correção proveniente dos fémions cuja contribuição majoritária provém dos quarks top e bottom [14] é

$$
\triangle \rho \approx \frac{3 G_{F}}{8 \pi^{2} \sqrt{2}}\left(m_{t}^{2}+m_{b}^{2}-2 \frac{m_{t}^{2} m_{b}^{2}}{m_{t}^{2}-m_{b}^{2}} \ln \frac{m_{t}^{2}}{m_{b}^{2}}\right) .
$$

A segunda contribuição ao parâmetro $\rho$ provém do fato de $g^{\prime} \neq 0$, dando origem as correções radiativas nas massas do $\mathrm{W}$ e $\mathrm{Z}$ devidas ao Higgs: 


$$
\triangle \rho=\frac{11 G_{F} M_{Z}^{2} \sin ^{2} \theta_{W}}{24 \sqrt{2} \pi^{2}} \ln \left(\frac{M_{H}^{2}}{M_{Z}^{2}}\right) .
$$

Apesar de essa correção depender apenas logaritmicamente na massa do Higgs sendo menor que a correção dos férmions, graças ao parâmetro $\rho$ ser determinado com grande acurácia experimental pode-se inferir destes limites à massa do Higgs. Na verdade desse parâmetro provém um dos maiores vínculos experimentais na massa deste bóson contribuindo sobremaneira na construção do gráfico (2.3), que será detalhado na próxima seção.

Fazendo um ajuste global aos dados do LEP conforme referência [10] tomando que:

$$
\begin{aligned}
114.4 \mathrm{GeV} & \leq M_{H} \leq 191 \mathrm{GeV} \\
M_{t} & =173.1 \pm 2.9 \mathrm{GeV} \\
\alpha_{s} & =0.1215 \pm 0.0017
\end{aligned}
$$

obtemos o valor experimental para $\rho$ muito próximo da unidade

$$
\rho_{\exp }=1.00002_{-0.0004}^{+0.0007}
$$

Esse fato impõe a qualquer teoria que aspire ser uma teoria além do MP que esta tenha inserida a simetria custodial de um modo aproximado, satisfazendo os vínculos experimentais. No modelo sem Higgs que estudaremos no próximo capítulo, veremos um modo de realizar tal feito.

\subsection{Resultados experimentais}

As interações eletrofracas do MP tem sido objeto de intenso escrutíneo experimental. Comprovando, por exemplo, as predições do modelo quanto as propriedades das correntes neutras, as propriedades dos bósons vetoriais $W^{ \pm}$e $Z$ dentre inúmeros outros aspectos. Para exemplificar, ressaltamos a medição do número de sabores para os neutrinos, que foram feitas nas "fábricas de Z's" do CERN e SLAC extraída da relação entre a largura experimental do $\mathrm{Z}$ em neutrinos $\left(\Gamma_{\text {invisivel }}\right)$ e a prevista pelo MP decaindo em uma só espécie de neutrino. 


$$
N_{\nu}=\Gamma_{\text {invisivel }} / \Gamma^{M P}\left(Z \rightarrow \nu_{i} \bar{\nu}_{i}\right)
$$

onde a $\Gamma_{\text {invisivel }}$ é obtida através da relação

$$
\Gamma_{\text {invisvel }}=\Gamma_{Z}-\Gamma_{\text {hadronico }}-3 \Gamma_{\text {leptonico }} .
$$

Resultando em $N_{\nu}=2.984 \pm 0.008$ [10] que está em pleno acordo com a existência

\begin{tabular}{|c|c|c|c|}
\hline & Measurement & Fit & 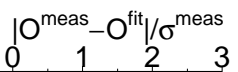 \\
\hline$\Delta \alpha_{\text {had }}^{(5)}\left(m_{z}\right)$ & $0.02758 \pm 0.00035$ & 0.02767 & \begin{tabular}{|l|l|l|} 
& & \\
\end{tabular} \\
\hline $\mathrm{m}_{\mathrm{z}}[\mathrm{GeV}]$ & $91.1875 \pm 0.0021$ & 91.1874 & \\
\hline$\Gamma_{\mathrm{z}}[\mathrm{GeV}]$ & $2.4952 \pm 0.0023$ & 2.4959 & b \\
\hline$\sigma_{\text {had }}^{0}[n b]$ & $41.540 \pm 0.037$ & 41.478 & \\
\hline $\mathrm{R}_{1}$ & $20.767 \pm 0.025$ & 20.742 & \\
\hline$A_{f b}^{0, I}$ & $0.01714 \pm 0.00095$ & 0.01643 & 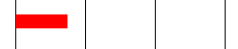 \\
\hline$A_{1}\left(P_{\tau}\right)$ & $0.1465 \pm 0.0032$ & 0.1480 & 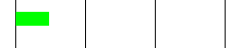 \\
\hline$R_{b}$ & $0.21629 \pm 0.00066$ & 0.21579 & 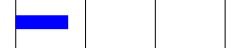 \\
\hline$R_{c}$ & $0.1721 \pm 0.0030$ & 0.1723 & 1 \\
\hline$A_{\mathrm{fb}}^{0, b}$ & $0.0992 \pm 0.0016$ & 0.1038 & \\
\hline$A_{\mathrm{fb}}^{0, \mathrm{c}}$ & $0.0707 \pm 0.0035$ & 0.0742 & \\
\hline$A_{b}$ & $0.923 \pm 0.020$ & 0.935 & \\
\hline$A_{c}$ & $0.670 \pm 0.027$ & 0.668 & 1 \\
\hline$A_{1}(S L D)$ & $0.1513 \pm 0.0021$ & 0.1480 & \\
\hline $\sin ^{2} \theta_{\text {eff }}^{\text {lept }}\left(Q_{f b}\right)$ & $0.2324 \pm 0.0012$ & 0.2314 & 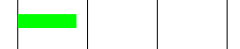 \\
\hline $\mathrm{m}_{\mathrm{w}}[\mathrm{GeV}]$ & $80.399 \pm 0.025$ & 80.378 & \\
\hline$\Gamma_{\mathrm{W}}[\mathrm{GeV}]$ & $2.098 \pm 0.048$ & 2.092 & 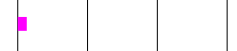 \\
\hline$m_{t}[\mathrm{GeV}]$ & $173.1 \pm 1.3$ & 173.2 & 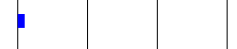 \\
\hline March 2009 & & & 1 \\
\hline
\end{tabular}
de 3 neutrinos $\nu_{e}, \nu_{\mu}, \nu_{\tau}$.

Figura 2.2: Comparação entre os resultados experimentais e o melhor ajuste global ao MP, ref. [19].

Como comprovação da concordância dos resultados experimentais com o previsto pelo MP expomos a figura (2.2), que possui na coluna da esquerda as medições de alguns observáveis obtidos no LEP, SLC e Tevatron. Na coluna da direita temos o melhor ajuste às predições do MP incluindo correções radiativas. Estes resul- 
tados mostram o impressionante ajuste do MP com os resultados experimentais, pelo menos até a escala de energia que provamos na atualidade.

Esses resultados experimentais também podem ser utilizados para prever a massa do Higgs, veja a figura (2.3). Nesta temos um ajuste feito pelo LEP Eletroweak Working Group [19] de $\triangle \chi^{2}$ em função da massa do Higgs utilizando os resultados experimentais do LEP, SLC e Tevatron. Esta figura indica uma massa para o Higgs

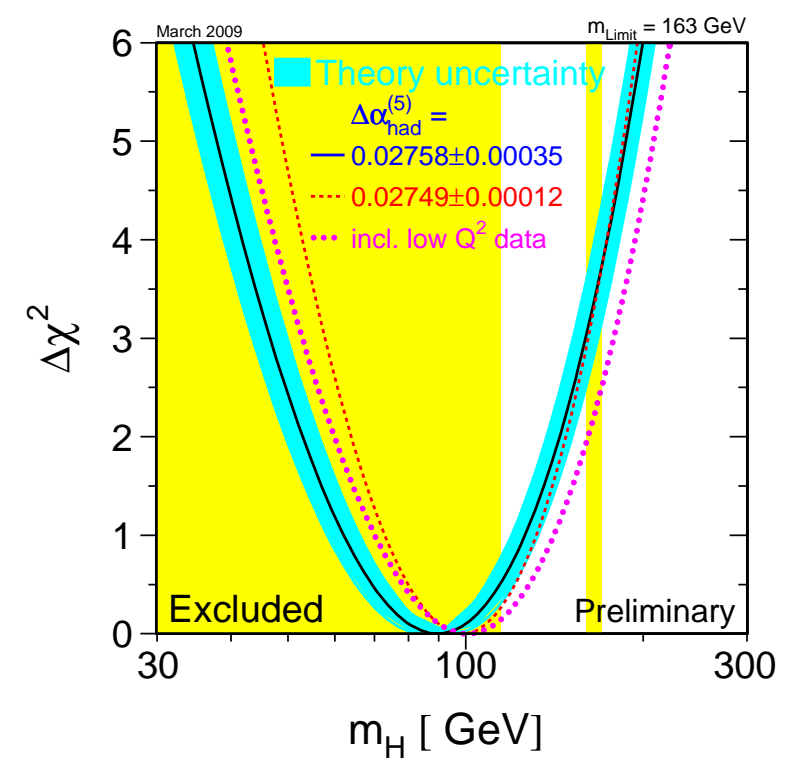

Figura 2.3: $\triangle \chi^{2}=\chi^{2}-\chi_{\min }^{2}$ em função da massa do Higgs obtido através de um ajuste global dos dados do LEP, SLC e Tevatron. A faixa colorida vertical indica regiões com $95 \%$ confiança de exclusão através de buscas diretas no LEP e Tevatron, ref. [19].

$$
M_{H}=90_{-27}^{+36} \mathrm{GeV} .
$$

Com

$$
M_{H}<163 \mathrm{GeV} \quad(95 \% \mathrm{CL}) .
$$

Contudo quando incluimos o vínculo de busca direta pelo Higgs no LEP, denotado pela faixa amarela, $M_{H}>114 \mathrm{GeV}(95 \% \mathrm{CL})$ temos que o limite superior sobe para 


$$
M_{H}<191 \mathrm{GeV} \quad(95 \% \mathrm{CL}) .
$$

Dado esse vínculo experimental temos que se o Higgs realmente existir nós estamos muito próximos de encontrá-lo.

\subsection{Limites teóricos na massa do Higgs}

Apesar do MP não fornecer um valor preciso para a massa do bósons de Higgs equação (2.71), argumentos teóricos que garantem a consistência do modelo podem nos ajudar colocando limites superior e inferior a essa massa.

\subsubsection{Unitariedade}

Podemos obter um vínculo superior à massa do Higgs simplesmente utilizando argumentos de unitariedade no espalhamento de bósons de gauge conforme [11, 14]. Para procedermos esta análise vamos tomar partido do Teorema da Equivalência [15], este nos diz que no regime de altas energias pode-se aproximar o espalhamento entre bósons vetoriaiscom polarização longitudinal pelo espalhamento entre bósons de Goldstone. Para entender essa equivalência devemos lembrar que os vetores de polarização dos bósons vetoriais $\mathrm{V}$ com momento $p^{\mu}=(E, 0,0,|\vec{p}|)$ são

$$
\epsilon_{T, 1}^{\mu}=\left(\begin{array}{c}
0 \\
1 \\
0 \\
0
\end{array}\right) ; \quad \epsilon_{T, 2}^{\mu}=\left(\begin{array}{c}
0 \\
0 \\
1 \\
0
\end{array}\right) ; \quad \epsilon_{L}^{\mu}=\frac{1}{m_{V}}\left(\begin{array}{c}
|\vec{p}| \\
0 \\
0 \\
E
\end{array}\right) \stackrel{E \gg M_{V}}{\longrightarrow} \frac{1}{M_{V}}\left(\begin{array}{c}
|\vec{p}| \\
0 \\
0 \\
|\vec{p}|
\end{array}\right)
$$

Portanto para espalhamentos a altas energias $\left(E \gg M_{V}\right)$ a principal contribuição provém da polarização longitudinal, $\left|\epsilon_{L}^{\mu}\right| \approx \frac{E}{M_{V}}$. Mas como já vimos na seção sobre Quebra Espontânea de Simetria, esse grau de liberdade longitudinal é devido justamente aos bósons de Goldstone.

Para calcularmos o espalhamento a altas energias de $W_{L}^{+} W_{L}^{-} \rightarrow W_{L}^{+} W_{L}^{-}$utilizando o Teorema da Equivalência devemos saber quais as regras de Feynman para os bósons de Goldstone. Com esse objetivo escrevemos o dubleto de Higgs como feito em [14] 


$$
\phi=\frac{1}{\sqrt{2}}\left(\begin{array}{c}
-\omega_{2}-i \omega_{1} \\
v+H+i \omega_{3}
\end{array}\right)
$$

e o potencial $V(\phi)$ do seguinte modo

$$
\begin{aligned}
V & =\mu^{2} \phi^{\dagger} \phi+\lambda\left(\phi^{\dagger} \phi\right)^{2} \\
& =\frac{M_{H}^{2}}{8 v^{2}}\left(\sum_{i=1}^{3} \omega_{i}^{2}\right)^{2}+\frac{M_{H}^{2}}{2 v} H \sum_{i=1}^{3} \omega_{i}^{2}+\ldots \\
& =\frac{M_{H}^{2}}{2 v^{2}}\left(\omega^{+} \omega^{-}\right)^{2}+\frac{M_{H}^{2}}{v} H \omega^{+} \omega^{-}+\ldots
\end{aligned}
$$

onde $\omega^{ \pm}=\left(\omega_{1} \mp i \omega_{2}\right) / \sqrt{2}$ são os bósons de Goldstone carregados. Assim conseguimos estabelecer as regras de Feynman que precisávamos como sendo $-2 i M_{H}^{2} / v^{2}$ para o vértice com 4 bósons de Goldstone e $-i M_{H}^{2} / v$ para o vértice com 2 bósons de Goldstone interagindo com o Higgs.

De posse dessas regras finalmente podemos calcular a amplitude desse espalhamento que possui os diagramas expressos na figura (2.4)

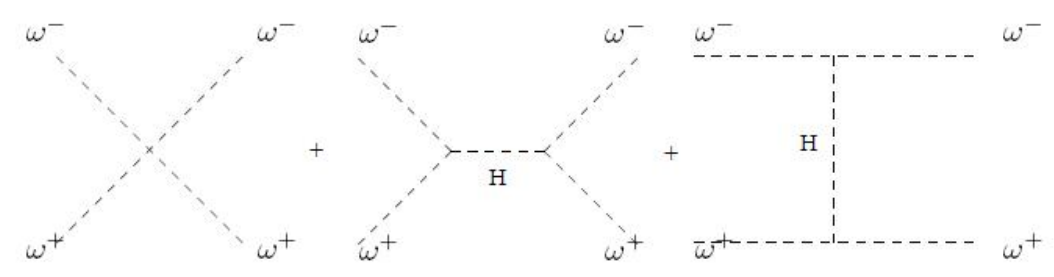

Figura 2.4: Diagramas que contribuem ao espalhamento $\omega^{+} \omega^{-} \rightarrow \omega^{+} \omega^{-}$.

$$
\mathcal{M}\left(W_{L}^{+} W_{L}^{-} \rightarrow W_{L}^{+} W_{L}^{-}\right)=-\frac{2 i M_{H}^{2}}{v^{2}}+\left(\frac{-i M_{H}^{2}}{v}\right)^{2} \frac{i}{s-M_{H}^{2}}+\left(\frac{-i M_{H}^{2}}{v}\right)^{2} \frac{i}{t-M_{H}^{2}}
$$

sendo $s=\left(p_{i n, 1}+p_{i n, 2}\right)^{2}$ o quadrado da energia do centro de massa do sistema e $t=\left(p_{\text {out }, 1}-p_{\text {in }, 1}\right)^{2}$.

Sabendo que a seção de choque diferencial e a amplitude estão relacionadas por

$$
\frac{d \sigma}{d \Omega}=\frac{|\mathcal{M}|^{2}}{64 \pi^{2} s}
$$

e que a amplitude em termos de ondas parciais 


$$
\mathcal{M}=16 \pi \sum_{l=0}^{\infty}(2 l+1) P_{l}(\cos \theta) a_{l},
$$

onde $P_{l}(x)$ são os polinômios de Legendre, podemos expressar a seção de choque através de

$$
\sigma=\frac{16 \pi}{s} \sum_{l=0}^{\infty}(2 l+1)\left|a_{l}\right|^{2} .
$$

Então se fizermos uso do Teorema óptico

$$
\sigma=\frac{1}{s} \operatorname{Im} \mathcal{M}(\theta=0)
$$

temos que para satisfazer (2.101) e (2.102) é necessário:

$$
\operatorname{Im}\left(a_{l}\right)=\left|a_{l}\right|^{2}
$$

Podendo ser reescrito como

$$
\left(\operatorname{Re} a_{l}\right)^{2}+\left(\operatorname{Im} a_{l}-\frac{1}{2}\right)^{2}=\frac{1}{4} .
$$

Lembrando que essa é apenas a expressão de um círculo no plano complexo de raio $1 / 2$ centrado no ponto $(0,1 / 2)$. Assim

$$
\left|\operatorname{Re}\left(a_{l}\right)\right| \leq \frac{1}{2}
$$

Vamos aplicar esse vínculo de unitariedade para a onda parcial $\mathrm{l}=0$ no limite $s \gg M_{H}^{2}$

$$
\begin{aligned}
a_{0}=\frac{1}{16 \pi s} \int_{-s}^{0} d t|\mathcal{M}| & =-\frac{1}{16 \pi s} \int_{-s}^{0} d t \frac{M_{H}^{2}}{v^{2}}\left(2+\frac{M_{H}^{2}}{s-M_{H}^{2}}+\frac{M_{H}^{2}}{t-M_{H}^{2}}\right) \\
& \approx-\frac{M_{H}^{2}}{16 \pi v^{2}}\left(2+\mathcal{O}\left(\frac{M_{H}^{2}}{s}\right)\right)
\end{aligned}
$$

Então com este resultado para $a_{0}$ e pelo vínculo de unitariedade pertubativa da teoria, conseguimos estabelecer um limite superior à massa do bóson de Higgs

$$
M_{H}^{2} \leq 4 \pi v^{2} \leq(872 \mathrm{GeV})^{2}
$$


Considerando outros processos além de $W_{L}^{+} W_{L}^{-} \rightarrow W_{L}^{+} W_{L}^{-}$obtemos um vínculo mais forte conforme [11]

$$
M_{H}^{2} \leq(780 \mathrm{GeV})^{2}
$$

Analisando a condição de unitariedade sob outra perspectiva $M_{H}^{2} \gg s$, ou seja, no caso em que não observa-se o Higgs, obtemos uma escala crítica de energia $\left(\sqrt{s_{c}}\right)$ onde espera-se ver alguma Física Além do Modelo Padrão. Considerando novamente o espalhamento $W_{L}^{+} W_{L}^{-} \rightarrow W_{L}^{+} W_{L}^{-}$obtemos

$$
a_{0}\left(\omega^{+} \omega^{-} \rightarrow \omega^{+} \omega^{-}\right) \stackrel{M_{H}^{2} \gg s}{\longrightarrow}-\frac{s}{32 \pi v^{2}} .
$$

Portanto para satisfazermos a condição de unitariedade (2.105) é necessário que

$$
\sqrt{s_{c}}<1.8 \mathrm{TeV}
$$

Usando outros espalhamentos esse limite pode ser reduzido para:

$$
\sqrt{s_{c}}<1.2 \mathrm{TeV}
$$

Este limite nos diz que não encontrando o Higgs do MP nós devemos ver nova física entre 1-2 TeV, fato que justifica toda a excitação da comunidade científica com a entrada em funcionamento do Large Hadron Collider (LHC), um colisor próton-próton de $\sqrt{s}=14 \mathrm{TeV}$.

\subsubsection{Trivialidade e Estabilidade do Vácuo}

Os limites que vamos impor agora à massa do Higgs provém de correções radiativas ao acoplamento quártico do Higgs $\lambda\left(Q^{2}\right)$. A equação do Grupo de Renormalização em ordem de 1-loop fornece conforme referência [14]:

$\frac{d \lambda}{d \log Q^{2}}=\frac{1}{16 \pi^{2}}\left[12 \lambda^{2}+6 \lambda \lambda_{t}^{2}-\frac{3}{2} \lambda\left(3 g^{2}+g^{\prime 2}\right)-3 \lambda_{t}^{2}+\frac{3}{16}\left(2 g^{4}+\left(g^{2}+g^{\prime 2}\right)^{2}\right)\right]+\ldots$

com $\lambda_{t}=\sqrt{2} m_{t} / v$ e as reticências indicam termos de ordens superiores, provenientes de fémions mais leves, que foram omitidos . O primeiro limite que vamos estudar é o de Trivialidade. Nesta condição $\lambda$ é muito grande e como $M_{H}^{2}=2 \lambda\left(v^{2}\right) v^{2}$ temos que $M_{H}$ também é. Esse limite permite considerarmos apenas o primeiro 
termo de (2.112)

$$
\frac{d \lambda}{d \log Q^{2}}=\frac{3}{4 \pi^{2}} \lambda^{2}
$$

Cuja solução é:

$$
\lambda\left(Q^{2}\right)=\frac{\lambda(v)}{1-\frac{3}{4 \pi^{2}} \lambda(v) \ln \left(\frac{Q^{2}}{v^{2}}\right)}
$$

Aumentando a escala de energia a partir de $Q \sim v$ vemos que $\lambda\left(Q^{2}\right)$ possui um pólo, denominado pólo de Landau, que nos fornece o valor máximo da escala de energia onde a teoria de perturbação funciona. Para não atingirmos o pólo impomos que $1 / \lambda\left(Q^{2}\right)>0$, obtendo assim um limite superior para a massa do Higgs

$$
M_{H}^{2}<\frac{8 \pi^{2} v^{2}}{3 \ln \left(\frac{\Lambda^{2}}{v^{2}}\right)}
$$

onde $\Lambda$ é o cutoff da teoria, ou seja, a escala onde aparece nova física.

Agora vamos abordar o limite de Estabilidade, este assegura $\lambda>0$ limitando o potencial do Higgs inferiormente. Na equação (2.112) os termos dominantes para $\lambda \sim 0$ são

$$
\frac{d \lambda}{d \log Q^{2}}=\frac{1}{16 \pi^{2}}\left[-3 \lambda_{t}^{2}+\frac{3}{16}\left(2 g^{4}+\left(g^{2}+g^{\prime 2}\right)^{2}\right)\right]
$$

com solução

$$
\lambda\left(Q^{2}\right)=\lambda\left(v^{2}\right)+\frac{1}{16 \pi^{2}}\left[-\frac{12 m_{t}^{2}}{v^{4}}+\frac{3}{16}\left(2 g^{4}+\left(g^{2}+g^{\prime 2}\right)^{2}\right)\right] \ln \left(\frac{Q^{2}}{v^{2}}\right) .
$$

Ao impor a condição $\lambda\left(Q^{2}\right)>0$ nos leva a um limite inferior à massa do Higgs

$$
M_{H}^{2}>\frac{v^{2}}{8 \pi^{2}}\left[\frac{12 m_{t}^{4}}{v^{4}}-\frac{3}{16}\left(2 g^{4}+\left(g^{2}+g^{\prime 2}\right)^{2}\right)\right] \ln \left(\frac{\Lambda^{2}}{v^{2}}\right),
$$

pois $\lambda\left(v^{2}\right)=M_{H}^{2} /\left(2 v^{2}\right)$.

Assim dos limites de Trivialidade e Estabilidade obtemos limites superior e inferior para a massa do Higgs em função do cutoff da teoria, como indica a figura (2.5). Supondo por exemplo que o MP é válido até $10^{16} \mathrm{GeV}$, então a massa do Higgs ficaria vinculada entre $130 \mathrm{GeV}$ e $170 \mathrm{GeV}$. Se esse vínculo não for satisfeito 
obtemos um sinal de física além do MP.

\subsection{O Problema da Hierarquia do Modelo Padrão}

Devido ao MP estar em perfeito acordo com os resultados experimentais uma questão que deve ser posta é: Se o Higgs do MP, com uma massa que satisfaça os vínculos teóricos e experimentais fosse descoberto, então isso garantiria não ver nenhum sinal de nova física até uma escala de energia muito alta? O que veremos nesta seção é que essa opção iria implicar em um ajuste fino dos parâmetros de um modo não natural, ou seja, sem nenhuma simetria que o assegure. E o mais empolgante, a escala de energia em que esse ajuste fino começa a ser importante é a escala a ser explorada pelo LHC 1-10 TeV, assim havendo nova física ela será vista em breve [16, 17].

Para entendermos melhor esse problema vamos analisar como aparecem as correções na massa dos férmions e dos bósons de gauge do MP e porque para estes não esperamos nenhum ajuste fino. Considerando primeiro a correção à massa dos férmions, temos que esta possui sempre a seguinte forma

$$
\delta m_{f} \propto m_{f} \ln \left(\frac{\Lambda^{2}}{m_{f}^{2}}\right),
$$

onde a massa do férmion renormalizada é dada pela soma da massa nua com a correção radiativa acima, $m_{f}^{r}=m_{f}+\delta m_{f}$. Então para os férmions temos que a correção da massa é sempre proporcional a sua massa nua, garantindo que as correções sejam pequenas. Este fato é uma consequência de ao tomarmos a massa do fémion nula ampliamos as simetrias da teoria, sendo a teoria neste limite invariante por transformações quirais $U(1)_{R} \otimes U(1)_{L}$

$$
\begin{aligned}
& \psi_{R} \rightarrow e^{i \theta_{R}} \psi_{R} \\
& \psi_{L} \rightarrow e^{i \theta_{L}} \psi_{L} .
\end{aligned}
$$

Como essa simetria é quebrada pelos acoplamentos de Yukawa, os quais são proporcionais a massa dos férmions, é garantido deste modo que as correções sejam proporcionais a $m_{f}$.

Situação análoga ocorre com as correções a massa dos bósons de gauge. Pois quando a massa desses bósons é nula a simetria de gauge é restaurada, fato que 
garante correções a massa dos bósons sempre proporcionais a massa nua dos mesmos.

Todavia quando consideramos a correção à massa do Higgs não existe nenhuma simetria que salvaguarde a proporcionalidade desta com a sua massa nua assegurando que esta seja naturalmente pequena. Pelo contrário, as correções são proporcionais ao quadrado do cutoff da teoria, exigindo assim um ajuste fino entre a massa nua e a correção, de modo a obtermos uma massa para o Higgs que satisfaça os limites teóricos e experimentais expostos nas seções anteriores.

Calculando os termos dominantes para essa correção obtemos conforme [11]:

$$
\delta M_{H}^{2} \approx \frac{\Lambda^{2}}{32 \pi^{2}}\left(-6 \lambda_{t}^{2}+\frac{1}{4}\left(9 g^{2}+3 g^{2}\right)+6 \lambda\right)
$$

Se tomarmos o cutoff da teoria em $\Lambda=10 \mathrm{TeV}$ temos que a massa do Higgs é dada por [17]

$$
\begin{aligned}
M_{H}^{2} & =M_{H, \text { tree }}^{2}+\delta M_{H}^{2} \\
& \approx M_{H, \text { tree }}^{2}+\frac{\Lambda^{2}}{32 \pi^{2}}\left(-6 \lambda_{t}^{2}+\frac{1}{4}\left(9 g^{2}+3 g^{\prime 2}\right)+6 \lambda\right) \\
& \approx M_{H, \text { tree }}^{2}-(100-10-5)(200 \mathrm{GeV})^{2}
\end{aligned}
$$

Donde vemos que o Problema da Hierarquia aparece claramente na escala $\Lambda=$ $10 \mathrm{TeV}$, pois para obtermos a massa do Higgs temos que fazer um ajuste fino de 1 parte em 100.

Para analisarmos qual é a escala de energia que estes cancelamentos começam a ficar importantes vamos reescrever a correção a massa do Higgs de modo que facilite essa análise [16]:

$$
\delta M_{H}^{2} \sim-\left(\frac{\Lambda}{0.7 T e V} 200 \mathrm{GeV}\right)^{2}
$$

Então para obtermos uma naturalidade devemos ter um cutoff menor que $1 \mathrm{TeV}$. Isso justifica o porquê de não termos visto física além do MP em colisores até hoje, pois esses possuem energias limitadas superiormente por $1 \mathrm{TeV}$ e somente para energias maiores que esta esse ajuste fino cresce em importância, então isso justifica a esperança de toda a comunidade científica para ver física nova com a entrada em funcionamento do LHC que possui $\sqrt{s}=14 \mathrm{TeV}$.

Combinando os limites teóricos temos os vínculos sobre a massa do Higgs ex- 


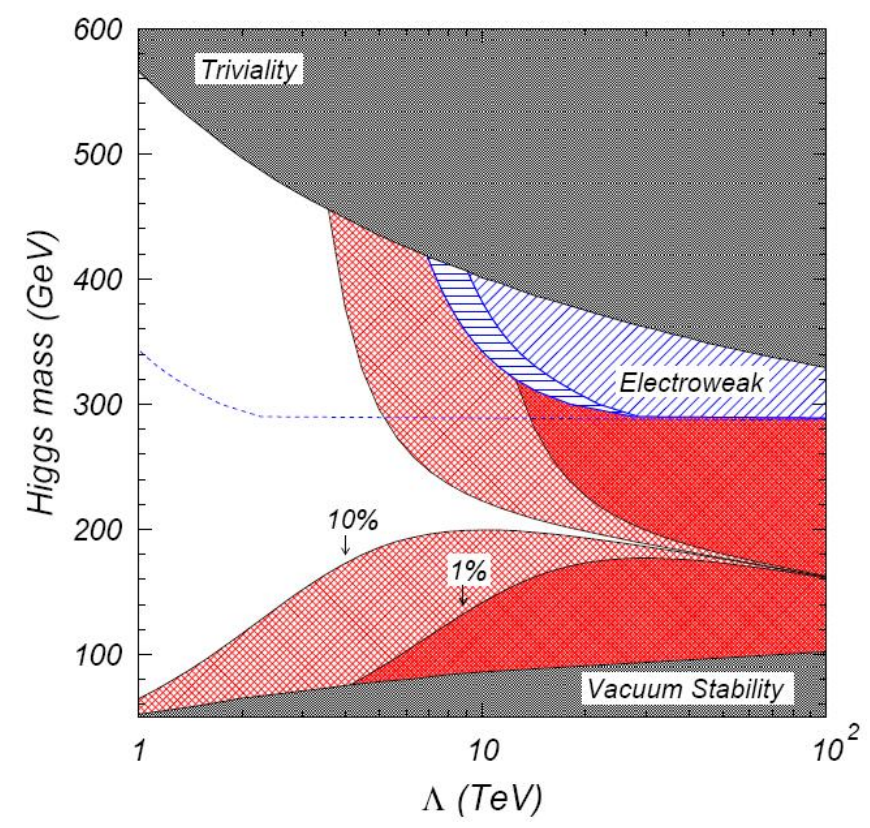

Figura 2.5: Regiões permitidas à $M_{H}$ em função do cutoff da teoria $\Lambda$. Nos extremos superior e inferior em preto temos os limites de Trivialidade e Estabilidade respectivamente. Nas partes em vermelho temos regiões de ajuste fino de $1 \%$ e $10 \%$ como indicado no gráfico. A região em branco é consistente com um ajuste fino menor que $10 \%$, ref. [18].

pressos na figura (2.5), onde nas partes em preto temos os limites de trivialidade e estabilidade e nas regiões em vermelho os limites de ajuste fino de $1 \%$ e $10 \%$. Além desses a referência [18] também apresenta na parte nomeada "Electroweak", região excluída por testes de precisão do MP referentes a extensões fortemente acopladas.

Combinando o gráfico (2.5) com o vínculo experimental (2.94) concluímos que o cutoff $\Lambda$ é sempre menor que $10 \mathrm{TeV}$, isso se considerarmos como limite para ver nova física um ajuste fino de 10\%. Logo o LHC deve nos proporcionar observar alguma física nova muito em breve. 


\section{Capítulo 3}

\section{Quebra espontânea de simetria por condições de contorno}

Vimos no capítulo anterior que o mecanismo de quebra de simetria do MP nos coloca diante de um ajuste fino já na escala de $1 \mathrm{TeV}$. Para contornar esse problema foram criados uma infinidade de modelos os quais utilizam algum tipo de mecanismo para efetuar a quebra espontânea da simetria eletrofraca. Um dos mecanismos mais recentes, cujo trabalho seminal data apenas de meados de 2003 [21], realiza a quebra da simetria por condições de contorno. Esses modelos ( Higgsless models) possuem ainda outra característica peculiar: a unitariedade do espalhamento de bósons vetoriais W e Z não é garantida pelo Higgs como no MP, mas sim por torres de bósons vetoriais massivos.

\subsection{Teorias de gauge em uma dimensão extra com- pacta: fixação de gauge e condições de con- torno}

Nesta seção, que possui um caráter introdutório, iremos obter quais são as condições de contorno consistentes para os campos de gauge em uma dimensão extra compacta $[0, \pi R]$. Nosso objetivo é partir de uma teoria em $5 \mathrm{D}$ e obter uma teoria efetiva em $4 \mathrm{D}$ ao fazer a redução dimensional

$$
S=\int d^{4} x \int_{0}^{\pi R} d y \mathcal{L}_{5 D}=\int d^{4} x \mathcal{L}_{4 D}
$$


Vamos denotar os campos de gauge como $A_{M}^{a}(x, y)$, onde $\mathrm{M}(0,1,2,3,5)$ é o índice de Lorentz ${ }^{1}, x$ é a coordenada nas 4 dimensões usuais e $y$ é a da dimensão extra. Além disso no princípio vamos adotar uma métrica plana com assinatura:

$$
g_{M N}=\operatorname{diag}(+1,-1,-1,-1,-1)
$$

Para as regiões da dimensão extra utilizaremos também uma nomenclatura especial: ao intervalo denominaremos bulk e aos extremos branas. Nesse estudo, por simplicidade, primeiramente vamos nos ater a uma teoria de gauge abeliana em 5D [20, 21, 22], cuja ação é dada por:

$$
S=\int d^{5} x\left(-\frac{1}{4} F_{M N} F^{M N}\right)=\int d^{5} x\left(-\frac{1}{4} F_{\mu \nu} F^{\mu \nu}-\frac{1}{2} F_{\mu 5} F^{\mu 5}\right)
$$

onde o tensor de intensidade $F_{M N}=\partial_{M} A_{N}-\partial_{N} A_{M}$ é expresso em termos do campo de gauge em 5D $A_{M}(x, y)$, o qual contém o campo vetorial em $4 \mathrm{D} A_{\mu}$ e o campo escalar em $4 \mathrm{D} A_{5}$. Analisando separadamente o termo adicional que depende do campo escalar em 4D

$$
\begin{aligned}
S_{A_{5}, A_{\mu}}= & \int d^{4} x \int_{0}^{\pi R} d y\left(-\frac{1}{2} F_{\mu 5} F^{\mu 5}\right) \\
= & -\frac{1}{2} \int d^{4} x \int_{0}^{\pi R} d y\left(\partial_{\mu} A_{5} \partial^{\mu} A^{5}+\partial_{5} A_{\mu} \partial^{5} A^{\mu}-2 \partial_{5} A_{\mu} \partial^{\mu} A^{5}\right) \\
= & -\frac{1}{2} \int d^{4} x \int_{0}^{\pi R} d y\left(-A_{5} \partial_{\mu} \partial^{\mu} A^{5}-A_{\mu} \partial_{5} \partial^{5} A^{\mu}-2 \partial_{5} A_{\mu} \partial^{\mu} A^{5}\right) \\
& -\frac{1}{2} \int d^{4} x\left[A_{\mu} \partial^{5} A^{\mu}+2 A_{5}\left(\partial_{\mu} A^{\mu}\right)\right]_{0}^{\pi R} .
\end{aligned}
$$

Note que ao integrarmos por partes em $x$ os termos se anulam nos extremos (infinito), contudo o mesmo não ocorre obrigatoriamente na dimensão extra por ser compacta, como explicitado na linha final. Na última igualdade dessa expressão identificamos o primeiro termo como o termo cinético do campo $A_{5}$, já o segundo futuramente veremos que pode ser identificado como termo de massa para os modos da teoria efetiva $\mathcal{L}_{4 D}$. Quanto ao terceiro termo dentro do parênteses, é um termo de mistura para os campos $A_{\mu}$ e $A_{5}$, portanto deve ser cancelado por uma escolha de gauge. Com tal intuito adicionamos a ação os termos de fixação de

\footnotetext{
${ }^{1}$ Quando nos referirmos as componentes $4 \mathrm{D}$ usuais $(0,1,2,3)$ usaremos índices gregos.
} 
gauge

$$
\begin{aligned}
S_{F G, \text { Bulk }}= & \int d^{4} x \int_{0}^{\pi R} d y-\frac{1}{2 \xi}\left(\partial_{\mu} A^{\mu}-\xi \partial_{5} A_{5}\right)^{2} \\
= & \int d^{4} x \int d y\left(-\frac{1}{2 \xi}\left(\partial_{\mu} A^{\mu}\right)^{2}-\partial_{\mu} A^{\mu} \partial_{5} A^{5}-\frac{\xi}{2} A_{5} \partial_{5} \partial^{5} A^{5}\right) \\
& +\left.\frac{\xi}{2} \int d^{4} x A_{5} \partial_{5} A^{5}\right|_{0} ^{\pi R},
\end{aligned}
$$

onde o segundo termo cancela exatamente o termo de mistura de (3.4).

Levando em conta os termos de fixação de gauge podemos obter a equação de movimento para o campo $A_{\mu}$ efetuando a variação da ação no Bulk:

$$
\left(\left(\partial_{\mu} \partial^{\mu}-\partial_{5}^{2}\right) g^{\mu \nu}-\left(1-\frac{1}{\xi}\right) \partial^{\mu} \partial^{\nu}\right) A_{\nu}(x, y)=0,
$$

já para o campo $A_{5}$ essa variação resulta na seguinte equação:

$$
\left(\partial_{\mu} \partial^{\mu}-\xi \partial_{5}^{2}\right) A_{5}(x, y)=0
$$

De posse dessas equações de movimento podemos analisar a dependência dos campos $A_{\mu}$ e $A_{5}$ com a dimensão extra. Seja

$$
A_{\mu}(x, y)=N \sum_{n} A_{\mu}^{(n)}(x) f_{n}(y)
$$

onde N é o fator de normalização, obtemos por separação de variáveis que:

$$
\begin{gathered}
\left(\left(\partial_{\mu} \partial^{\mu}+m_{n}^{2}\right) g^{\mu \nu}-\left(1-\frac{1}{\xi}\right) \partial^{\mu} \partial^{\nu}\right) A_{\nu}^{(n)}(x)=0 \\
\left(\partial_{5}^{2}+m_{n}^{2}\right) f_{n}(y)=0
\end{gathered}
$$

Donde temos que a dependência na dimensão extra do campo $A_{\mu}(x, y)$ é uma soma de senos e cossenos e as massas dos campos vetoriais em $4 \mathrm{D} A_{\mu}^{(n)}(x)$ são os autovalores da equação (3.10).

Para o campo $A_{5}(x, y)$ procedemos analogamente realizando a seguinte decomposição 


$$
A_{5}(x, y)=N \sum_{n} A_{5}^{(n)}(x) f_{n}(y)
$$

assim (3.7) resulta por separação de variáveis em:

$$
\begin{array}{r}
\left(\partial_{\mu} \partial^{\mu}+\xi m_{n}^{2}\right) A_{5}^{(n)}(x)=0 \\
\left(\partial_{5}^{2}+m_{n}^{2}\right) f_{n}(y)=0
\end{array}
$$

Observe que a massa do campo escalar em 4D $A_{5}^{(n)}(x)$ depende de gauge $m_{A_{5}^{(n)}}^{2}=$ $\xi m_{n}^{2}$. Além disso temos que os campos $A_{\mu}(x, y)$ e $A_{5}(x, y)$ compartilham da mesma equação de movimento para suas funções de onda $f_{n}(y)$.

Tendo em vista este fato podemos decompor o campo $A_{M}$ em modos de KaluzaKlein $(\mathrm{KK}) A_{M}^{(n)}$ e $\hat{A}_{M}^{(n)}$ como segue:

$$
A_{M}(x, y)=A_{M}^{(0)}(x)+\sum_{n=1}^{\infty}\left(A_{M}^{(n)}(x) \cos \left(m_{n} y\right)+\hat{A}_{M}^{(n)}(x) \sin \left(m_{n} y\right)\right)
$$

esta decomposição finalmente nos permite interpretar o segundo termo de (3.4), pois

$$
\partial_{5}^{2} A_{\mu}(x, y)=-\sum_{n=1}^{\infty} m_{n}^{2}\left(A_{\mu}^{(n)}(x) \cos \left(m_{n} y\right)+\hat{A}_{\mu}^{(n)}(x) \sin \left(m_{n} y\right)\right)
$$

nos proporciona termos de massa para os KK na teoria efetiva $\mathcal{L}_{4 D}$ que obtemos integrando a dimensão extra como expresso na equação (3.1). A teoria efetiva $4 \mathrm{D}$ terá em princípio duas torres de fótons massivos com massas inversamente proporcionais ao tamanho da dimensão extra ${ }^{2}$ como indicado na figura (3.1), onde a existência de cada torre dependerá das condições de contorno na dimensão extra.

Observe que esse mecanismo não gera massa para o modo $A_{\mu}^{(0)}(x)$. Sendo que a existência desse passa a ser um indício da manutenção da simetria original $U(1)$, apesar de todos os demais modos serem massivos. Neste ponto fica clara a importância das condições de contorno, pois este modo irá existir se impormos Neumann nas duas branas $\left.\partial_{5} A_{\mu}\right|_{0, \pi R}=0$ e não irá existir desde que impusermos

\footnotetext{
${ }^{2}$ Essa dependência da massa com o tamanho da dimensão extra será obtida após impormos as condições de contorno.
} 


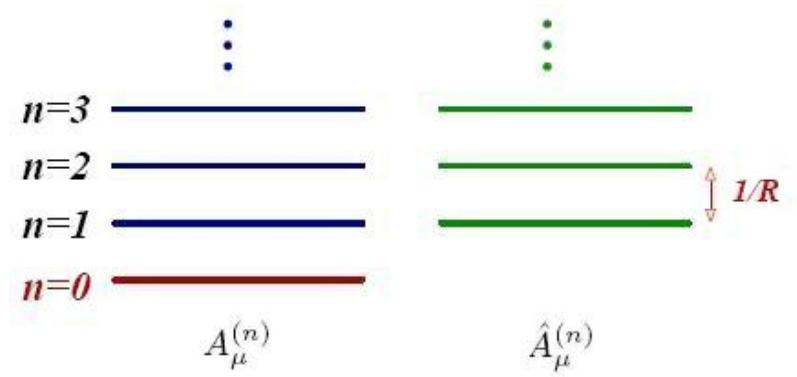

Figura 3.1: Espectro de massa dos modos de Kaluza-Klein.

Dirichlet $\left.A_{\mu}\right|^{0, \pi R}=0$ em pelo menos uma das branas. Todavia ainda devemos checar quais condições de contorno podemos aplicar de modo consistente, ou seja, respeitando as simetrias da teoria.

Dando prosseguimento a análise obtivemos que as massas dos modos $A_{5}^{(n \neq 0)}$ são dadas por $m_{A_{5}^{(n)}}^{2}=\xi m_{n}^{2}$, dependendo da escolha de gauge $\xi$. Essa dependência nos possibilitará desacoplar todos os modos $A_{5}^{(n)}(x) \operatorname{com} n \neq 0$ simplesmente adotando o gauge unitário $\xi \rightarrow \infty$, pois neste caso esses modos apresentarão massa infinita na teoria efetiva $4 \mathrm{D}$. Observe no entanto que o modo $n=0$ do campo $A_{5}$ ainda poderá existir, dependendo das condições de contorno possíveis.

Agora vamos retomar a expressão (3.4), pois ainda nos resta eliminar o termo de mistura $A_{5} \partial_{\mu} A^{\mu}$ que está nas branas. Para tal adicionamos também um termo de fixação de gauge nas branas:

$$
S_{F G, \text { branas }}=-\left.\frac{1}{2 \xi_{\text {brana }}} \int d^{4} x\left(\partial_{\mu} A^{\mu} \pm \xi_{\text {brana }} A_{5}\right)^{2}\right|_{0, \pi R}
$$

onde o sinal "-" remete a $y=0$ e o sinal "+" a $y=\pi R$, note que $\xi$ e $\xi_{\text {brana }}$ não precisam ser iguais.

Visando saber quais são as possíveis condições de contorno definimos $S_{\text {brana }}$ como a soma de todos os termos sobre as branas em (3.4), (3.5), (3.16) e fazemos a variação:

$\delta S_{\text {brana }}=\int d^{4} x\left[\left.\left(\partial_{5} A^{\mu}+\frac{1}{\xi_{\text {brana }}} \partial_{\nu} \partial^{\mu} A^{\nu}\right) \delta A_{\mu}\right|_{0, \pi R}+\left.\left(\xi \partial_{5} A_{5} \pm \xi_{\text {brana }} A_{5}\right) \delta A_{5}\right|_{0, \pi R}\right]=0$

Então as condições de contorno em um gauge arbitrário $\xi$ e $\xi_{\text {brana }}$ são

$$
\partial_{5} A^{\mu}+\frac{1}{\xi_{\text {brana }}} \partial_{\nu} \partial^{\mu} A^{\nu}=0, \quad \xi \partial_{5} A_{5} \pm \xi_{\text {brana }} A_{5}=0
$$


Que no gauge unitário $\xi_{\text {brana }} \rightarrow \infty$ se reduz a

$$
\left.\partial_{5} A^{\mu}\right|_{0, \pi R}=0,\left.\quad A_{5}\right|_{0, \pi R}=0 .
$$

A condição de contorno tipo Neumann para o campo $A_{\mu}$ impõe que este possua modo KK $n=0$, todavia a condição tipo Dirichlet para o campo $A_{5}$ obriga que este não tenha modo $n=0$. Assim teremos na teoria efetiva $4 \mathrm{D}$ um fóton não massivo $A_{\mu}^{(0)}$ e nenhum modo do campo $A_{5}$. Donde concluímos que não podemos quebrar a simetria $U(1)$ por condições de contorno se não introduzirmos nenhum novo ingrediente nessa teoria.

\subsubsection{A origem da massa para os KK's}

Visando elucidar esse mecanismo de geração de massa para os KK $A_{\mu}^{(n \neq 0)}$ vamos reformular a escolha de gauge de um modo mais simples, essa simplificação deixará explícito o fato que os campos $A_{5}^{(n \neq 0)}$ serão absorvidos como componente longitudinal dos campos vetoriais. Neste sentido esse mecanismo será análogo ao MP, onde os bósons de Goldstone são absorvidos como componente longitudinal dos bósons de gauge gerando massa para estes. Para mostrarmos tal analogia tomaremos partido do conhecimento sobre as condições de contorno dos campos $A_{\mu}$ e $A_{5}$ (3.19), as quais implicam na seguinte decomposição em modos de Kaluza-Klein:

$$
\begin{aligned}
& A_{\mu}(x, y)=\frac{1}{\sqrt{\pi R}} A_{\mu}^{(0)}+\sqrt{\frac{2}{\pi R}} \sum_{n=1}^{\infty} A_{\mu}^{(n)}(x) \cos \left(\frac{\left(n-\frac{1}{2}\right) y}{R}\right) \\
& A_{5}(x, y)=\sqrt{\frac{2}{\pi R}} \sum_{n=1}^{\infty} A_{5}^{(n)} \sin \left(\frac{\left(n-\frac{1}{2}\right) y}{R}\right)
\end{aligned}
$$

Tendo em vista essa decomposição dos campos em modos de Kaluza-Klein e a equação (3.1) obtemos a lagrangiana efetiva 4D:

$$
\begin{aligned}
\mathcal{L}_{4 D} & =\int_{0}^{\pi R} d y\left(-\frac{1}{4} F_{M N} F^{M N}\right)=\int_{0}^{\pi R} d y\left(-\frac{1}{4} F_{\mu \nu} F^{\mu \nu}-\frac{1}{2} F_{\mu 5} F^{\mu 5}\right) \\
& =\int_{0}^{\pi R} d y\left(-\frac{1}{4} F_{\mu \nu} F^{\mu \nu}-\frac{1}{2}\left(\partial_{\mu} A_{5} \partial^{\mu} A^{5}+\partial_{5} A_{\mu} \partial^{5} A^{\mu}-2 \partial_{\mu} A_{5} \partial^{5} A^{\mu}\right)\right) \\
& =-\frac{1}{4} \sum_{n=0}^{\infty} F_{\mu \nu}^{(n)} F^{(n) \mu \nu}+\frac{1}{2} \sum_{n=1}^{\infty}\left(\partial_{\mu} A_{5}^{(n)}+\left(\frac{n-\frac{1}{2}}{R}\right) A_{\mu}^{(n)}\right)^{2}
\end{aligned}
$$


Lembrando que a simetria de gauge $U(1)$ da teoria 5D, $A_{M} \rightarrow A_{M}+\partial_{M} \Lambda(x, y)$, pode ser posta em termos dos modos de Kaluza-Klein como

$$
\begin{array}{ll}
A_{\mu}^{(n)} \rightarrow A_{\mu}^{(n)}+\partial_{\mu} \Lambda^{(n)}(x), & n \in \mathbb{N} \\
A_{5}^{(n)} \rightarrow A_{5}^{(n)}-\left(\frac{n-\frac{1}{2}}{R}\right) \Lambda^{(n)}(x), & n \in \mathbb{N}^{*},
\end{array}
$$

invariância que podemos checar diretamente de (3.22). Temos que o gauge unitário pode ser fixado simplesmente impondo para $n \neq 0$ :

$$
\Lambda^{(n)}=\frac{R}{n-\frac{1}{2}} A_{5}^{(n)}, \quad n \in \mathbb{N}^{*}
$$

que implica em:

$$
\mathcal{L}_{4 D}=-\frac{1}{4} \sum_{n=0}^{\infty} F_{\mu \nu}^{(n)} F^{(n) \mu \nu}+\frac{1}{2} \sum_{n=1}^{\infty}\left(\frac{n-\frac{1}{2}}{R}\right)^{2} A_{\mu}^{(n)} A^{(n) \mu}
$$

donde conclui-se que o grau de liberdade longitudinal dos bósons vetoriais KK é fornecido justamente pelo $A_{5}^{(n \neq 0)}$ nesse "mecanismo de Higgs 5D", onde o modo $A_{5}^{(n \neq 0)}$ faz o papel de bóson de Goldstone do $A_{\mu}^{(n \neq 0)}$.

Desse mecanismo resulta um espectro de massa para os bósons vetoriais com o modo $n=0$ não massivo e os demais modos com massa:

$$
m_{n}=\frac{n-\frac{1}{2}}{R}, \quad n \in \mathbb{N}^{*} .
$$

Esse melhor entendimento do mecanismo de geração de massa reafirma a necessidade de introdução de um novo elemento na teoria se pretendermos ter todos os $\operatorname{modos} A_{\mu}^{(n)}$ massivos.

\subsection{Teorias de Gauge com escalares nas branas}

Visando ampliar as possibilidades para as condições de contorno, vamos analisar as modificações ao inserir um campo escalar nas branas com lagrangiana

$$
\mathcal{L}_{i}=\left|D_{\mu} \Phi_{i}\right|^{2}-\lambda_{i}\left(\left|\Phi_{i}\right|^{2}-\frac{1}{2} v_{i}^{2}\right)^{2}
$$


onde o índice $\mathrm{i}=1,2$ denota as branas $y=0$ e $y=\pi R$ respectivamente [20]. Tendo em vista que o vev é não nulo parametrizamos esses campos como no capítulo anterior, em termos do Higgs $h$ e do bóson de Goldstone $\pi$ :

$$
\Phi_{i}=\frac{1}{\sqrt{2}}\left(v_{i}+h_{i}\right) e^{i \pi_{i} / v_{i}}
$$

Então expandindo até a ordem quadrática nos campos obtemos nesse caso que

$$
\begin{aligned}
\mathcal{L}_{4 D}= & \int_{0}^{\pi R} d y\left(-\frac{1}{4} F_{\mu \nu} F^{\mu \nu}-\frac{1}{2}\left(\partial_{\mu} A_{5}\right)^{2}-\frac{1}{2}\left(\partial_{5} A_{\mu}\right)^{2}+\partial_{\mu} A_{5} \partial^{5} A^{\mu}\right) \\
& +\left(\frac{1}{2}\left(\partial_{\mu} h_{1}\right)^{2}-\frac{1}{2} \lambda_{1} v_{1}^{2} h_{1}^{2}+\frac{1}{2}\left(\partial_{\mu} \pi_{1}-v_{1} A_{\mu}\right)^{2}\right)_{y=0} \\
& +\left(\frac{1}{2}\left(\partial_{\mu} h_{2}\right)^{2}-\frac{1}{2} \lambda_{2} v_{2}^{2} h_{2}^{2}+\frac{1}{2}\left(\partial_{\mu} \pi_{2}-v_{2} A_{\mu}\right)^{2}\right)_{y=\pi R} .
\end{aligned}
$$

De modo análogo a seção anterior temos que introduzir termos de fixação de gauge para cancelar os termos de mistura $\left(A_{5}-A_{\mu}\right)$ e $\left(\pi-A_{\mu}\right)$

$$
\begin{aligned}
\mathcal{L}_{F G}= & -\frac{1}{2 \xi} \int_{0}^{\pi R} d y\left(\partial_{\mu} A^{\mu}-\xi \partial_{5} A_{5}\right)^{2}-\left(\frac{1}{2 \xi_{1}}\left(\partial_{\mu} A^{\mu}+\xi_{1}\left(v_{1} \pi_{1}-A_{5}\right)\right)^{2}\right)_{y=0} \\
& -\left(\frac{1}{2 \xi_{2}}\left(\partial_{\mu} A^{\mu}+\xi_{2}\left(v_{2} \pi_{2}+A_{5}\right)\right)^{2}\right)_{y=\pi R}
\end{aligned}
$$

Quanto às condições de contorno do campo $A_{\mu}$, as obtemos efetuando a variação da ação sobre as branas, que no gauge unitário resulta em

$$
\left.\left(\partial_{5} \mp v_{1,2}^{2}\right) A_{\mu}\right|^{0, \pi R}=0 .
$$

Deste modo conclui-se que a adição do campo escalar nas branas nos permite condições de contorno tipo Dirichlet $\left.A_{\mu}\right|_{i}=0$ se $v_{i} \rightarrow \infty$. Assim obtemos a possibilidade de quebrar a simetria em uma das branas, pois como já destacado se impormos a condição tipo Dirichlet, o campo do fóton não terá o modo zero, que é um indício de quebra de simetria tendo em vista que o primeiro modo existente agora também será massivo.

Cumpre ainda salientar que no limite $v_{i} \rightarrow \infty$ os campos $h_{i}, \pi_{i}$ desacoplam do campo de gauge, pois eles são não nulos apenas onde o campo de gauge é nulo. Então o efeito de um campo escalar localizado na brana quando tomamos seu vev 
tendendo a infinito é simplesmente expulsar o campo de gauge da brana e impor a inexistência de KK's não massivos para o campo vetorial.

Podemos ir ainda mais longe quanto aos escalares. Começando pelo campo do Higgs $h_{i}$ constatamos na equação (3.30) que se tomarmos partido da independência do parâmetro $\lambda_{i}$ podemos desacoplar completamente $h_{i}$ da teoria dando uma massa suficientemente alta para ele sem influenciar os demais campos. Agora quanto aos campos $\pi$ e $A_{5}$ podemos obter as condições de contorno desses fazendo a variação da ação sobre as branas assim como fizemos para a teoria de gauge pura. Deste procedimento obtemos que $\pi_{i}=A_{5} / v_{i}$ no gauge unitário, portanto $\pi_{i}$ desacopla da teoria no limite $v_{i} \rightarrow \infty$ por ser nulo. Já no que concerne o escalar $A_{5}$ temos que esse muda sua condição de contorno de Dirichlet na equação (3.19) para Neumann $\left.\partial_{5} A_{5}\right|_{0, \pi R}=0$, possibilitando assim a existência do modo zero não massivo para ele se impormos Neumann nas duas branas.

Como vimos na seção anterior todos os modos dos campos vetoriais com $n>$ 0 tornam-se massivos ao absorver os modos dos campos escalares, todavia $A_{\mu}^{(0)}$ continuou não massivo, fato que poderiamos conjecturar explicar pela inexistência do campo $A_{5}^{(0)}$ naquele caso. Como vimos nos casos sem e com escalar na brana, teremos sempre condições de contorno opostas para os campos $A_{\mu}$ e $A_{5}$, sempre trocando Dirichlet por Neumann. Deste modo conclui-se na impossibilidade de gerar os modos $A_{5}^{(0)}$ e $A_{\mu}^{(0)}$ concomitantemente. Implicando que o $A_{5}^{(0)}$ não pode ser absorvido por uma escolha de gauge e permanecerá no espectro. Então se desejarmos evitar a existência de escalares no espectro (como faremos adiante) teremos que impor ao campo vetorial $A_{\mu}$ condições de contorno tipo NeumannNeumann ou Dirichlet-Neumann.

\subsection{Toy models}

Como o intuito de ganharmos mais intuição nesse mecanismo de quebra de simetria vamos abordar três exemplos. Os dois primeiros possuem apenas um caráter didático, contudo no último será delineada a estrutura básica dos principais Higgsless Models.

\subsubsection{Geração de massa para o fóton}

Considerando uma teoria de gauge $U(1)$ no bulk definamos as condições de contorno como Dirichlet em $y=0$ e Neumann em $y=\pi R$. 


$$
\left\{\begin{array}{l}
\left.A_{\mu}\right|_{y=0}=0 \\
\left.\partial_{5} A_{\mu}\right|_{y=\pi R}=0
\end{array}\right.
$$

Resolvendo a equação de movimento (3.10) e impondo essas condições de contorno podemos expressar o campo vetorial em modos de Kaluza-Klein como

$$
A_{\mu}=\sqrt{\frac{2}{\pi R}} \sum_{n=1}^{\infty} \hat{A}_{\mu}^{(n)}(x) \sin \left(\frac{2 n-1}{2 R} y\right) .
$$

Tendo em vista que todos os campos escalares desacoplam com as condições de contorno (3.33) se fixarmos o gauge unitário, como visto anteriormente, podemos reescrever (3.30) e efetuar a redução dimensional como segue:

$$
\begin{aligned}
\mathcal{L}_{4 D} & =\int_{0}^{\pi R}\left(-\frac{1}{4} F_{\mu \nu} F^{\mu \nu}-\frac{1}{2}\left(\partial_{5} A_{\mu}\right)^{2}\right) \\
& =\sum_{n=1}^{\infty}\left(-\frac{1}{4} \hat{F}_{\mu \nu}^{(n)}(x) \hat{F}^{(n) \mu \nu}(x)+\frac{1}{2}\left(\frac{2 n-1}{2 R}\right)^{2} \hat{A}_{\mu}^{(n)}(x) \hat{A}^{(n) \mu}(x)\right)
\end{aligned}
$$

com $\hat{F}_{\mu \nu}=\partial_{\mu} \hat{A}_{\nu}-\partial_{\nu} \hat{A}_{\mu}$. Observe que temos somente uma torre de Kaluza-Klein da figura (3.1), a qual possui todos os modos massivos

$$
m_{n}=\frac{2 n-1}{2 R}, \quad n \in \mathbb{N}^{*} .
$$

Vale ressaltar que ao adotar condições de contorno tipo Dirichlet-Neumann conseguimos obter todos os modos massivos, fato que não tinhamos conseguido quando só podiamos impor Neumann-Neumann nas branas como em (3.26).

\subsubsection{Geração de massa para os bósons de $S U(2)$}

Neste exemplo começamos com uma teoria de gauge $S U(2)$ no bulk, figura (3.2), e por condições de contorno o quebramos no grupo $U(1)$ [20]. Para obter tal resultado as condições de contorno impostas são:

$$
\begin{cases}\left.\partial_{5} A_{\mu}^{1,2}\right|_{y=0}=0, & \left.A_{\mu}^{1,2}\right|_{y=\pi R}=0 \\ \left.\partial_{5} A_{\mu}^{3}\right|_{y=0}=0, & \left.\partial_{5} A_{\mu}^{3}\right|_{y=\pi R}=0\end{cases}
$$

Então ao resolver a equação de movimento (3.10) e impor as condições de contorno podemos decompor os campos $A_{\mu}^{a}$ em modos de Kaluza-Klein como segue: 


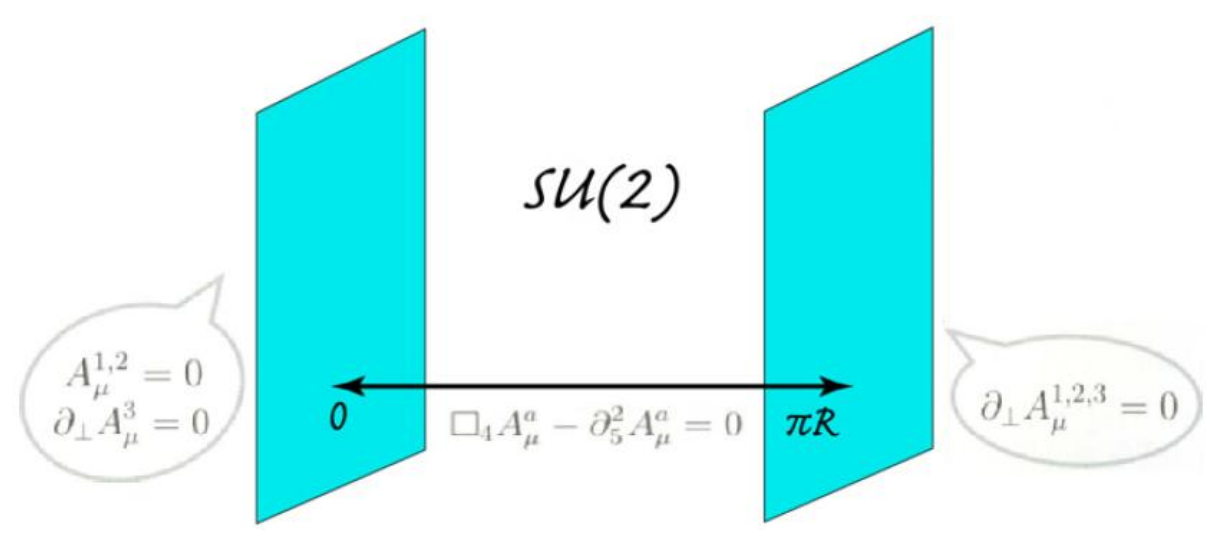

Figura 3.2: Quebra da simetria $S U(2) \rightarrow U(1)$ por condições de contorno.

$$
\begin{aligned}
& A_{\mu}^{1}(x, y)=\sum_{n=1}^{\infty} \frac{1}{\sqrt{\pi R}} \sin \left(\frac{(2 n-1) y}{2 R}\right)\left(W_{\mu}^{+(n)}(x)+W_{\mu}^{-(n)}(x)\right) \\
& A_{\mu}^{2}(x, y)=\sum_{n=1}^{\infty} \frac{i}{\sqrt{\pi R}} \sin \left(\frac{(2 n-1) y}{2 R}\right)\left(W_{\mu}^{+(n)}(x)+W_{\mu}^{-(n)}(x)\right) \\
& A_{\mu}^{3}(x, y)=\sum_{n=0}^{\infty} \sqrt{\frac{2}{2^{\delta_{n, 0}} \pi R}} \cos \left(\frac{n y}{R}\right) \gamma_{\mu}^{(n)}(x)
\end{aligned}
$$

Dessa decomposição temos as seguintes massas para os KK's $M_{\gamma^{(n)}}=n / R$ e $M_{W^{(n)}}=(2 n-1) /(2 R)$. Note que esse espectro possui uma semelhança com o MP desde que identifiquemos o fóton com o bóson não massivo $\gamma^{(0)}$, o $\mathrm{Z}$ com o primeiro estado excitado $\gamma^{(1)}$ (por este também ser neutro), e os bósons carregados $W^{ \pm}$com $W^{ \pm(1)}$. Contudo essa tentativa ingênua de obter o modelo padrão de um modelo tão simples não é bem sucedida, pois implicaria na razão

$$
\frac{M_{Z}}{M_{W}}=2
$$

a qual violaria a simetria custodial, conforme constatamos na equação (2.72). Além disso esse modelo impõe a existência de modos de Kaluza-Klein com massa da ordem de $250 \mathrm{GeV}$, dado que

$$
\frac{M_{Z^{\prime}}}{M_{Z}}=2, \quad \frac{M_{W^{\prime}}}{M_{W}}=3
$$

o que não é condizente com os resultados experimentais. 


\subsubsection{Higgsless model com simetria custodial}

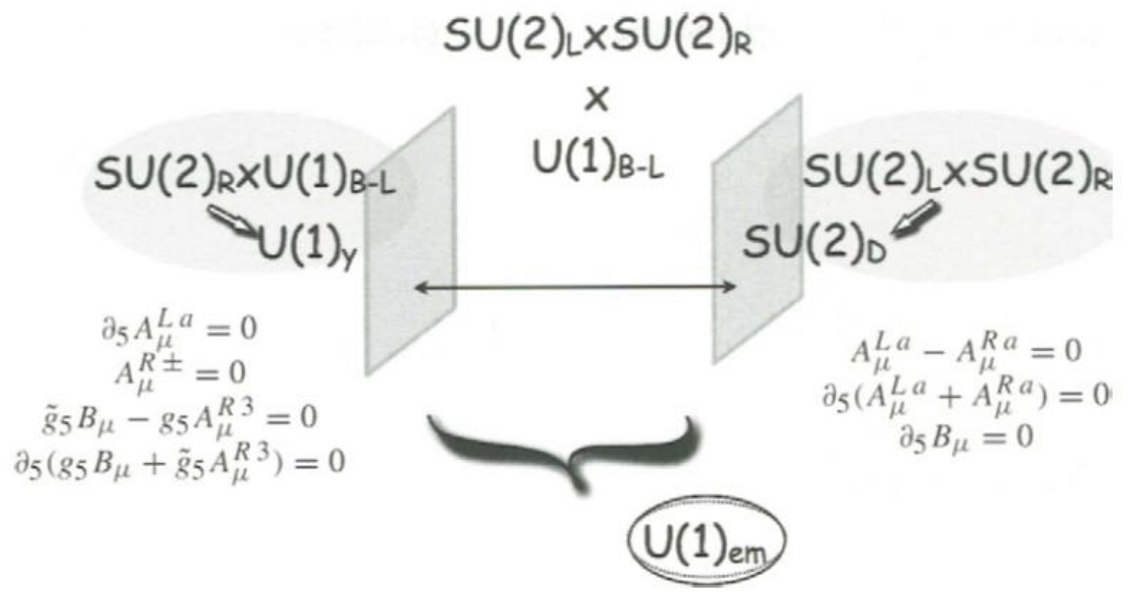

Figura 3.3: Quebra de simetria por condições de contorno no Higgsless model com dimensão extra plana, ref. [11].

O exemplo anterior nos lembra que devemos de algum modo inserir as simetrias do MP, em especial a simetria custodial $S U(2)_{D}$ em uma possível extensão a ele. Tendo em vista isso Csáki et al. [21] propõem um modelo com um grupo de simetria maior no bulk $S U(2)_{L} \otimes S U(2)_{R} \otimes U(1)_{B-L}$ de modo que ao quebrarmos essa simetria por condições de contorno evidenciemos as simetrias que deseja-se proteger $^{3}$. Com este objetivo em vista efetua-se a quebra na brana $y=0 \mathrm{em}$ $S U(2)_{L} \otimes S U(2)_{R} \rightarrow S U(2)_{D}$ mantendo a simetria $S U(2)_{D} \otimes U(1)_{B-L}$ e na brana $y=\pi R$ em $S U(2)_{R} \otimes U(1)_{B-L} \rightarrow U(1)_{Y}$ mantendo a simetria $S U(2)_{L} \otimes U(1)_{Y}$, conforme ilustrado na figura (3.3).

Designaremos por $A_{M}^{(L)}, A_{M}^{(R)}$ e $B_{M}$ os bósons de gauge $5 \mathrm{D}$ dos grupos $S U(2)_{L}$, $S U(2)_{R}$ e $U(1)_{B-L}$ respectivamente, com constantes de acoplamento $g_{5 L}, g_{5 R}$ e $\tilde{g}_{5}$. Para implementarmos a quebra na brana $y=\pi R$ levaremos em conta a combinação $\left(c A_{\mu}^{a(R)}+s A_{\mu}^{a(L)}\right)$ e $\left(-s A_{\mu}^{a(R)}+c A_{\mu}^{a(L)}\right)$, onde $c \equiv g_{5 L}$ e $s \equiv g_{5 R}$ fazem a mistura dos bósons de gauge. A combinação positiva corresponde a parte não quebrada $S U(2)_{D}$ de modo a termos na brana $y=\pi R$ as seguintes condições de contorno:

$$
\left\{\begin{array}{l}
\left.\partial_{5}\left(g_{5 R} A_{\mu}^{a(L)}+g_{5 L} A_{\mu}^{a(R)}\right)\right|_{y=\pi R}=0,\left.\quad \partial_{5} B_{\mu}\right|_{y=\pi R}=0 \\
\left.\left(-g_{5 R} A_{\mu}^{a(R)}+g_{5 L} A_{\mu}^{a(L)}\right)\right|_{y=\pi R}=0
\end{array}\right.
$$

Quanto à brana $y=0$, como denotado na figura (3.3) visamos efetuar a quebra

\footnotetext{
${ }^{3}$ A simetria $U(1)_{B-L}$ é inserida para evitar anomalias devidas a violação do número B-L por efeitos quânticos.
} 
$S U(2)_{R} \otimes U(1)_{B-L} \rightarrow U(1)_{Y}$, para isso vamos definir o bóson de gauge associado ao grupo resultante $U(1)_{Y}$ como a mistura $\left(c A_{\mu}^{3(R)}+s B_{\mu}\right)$ e para a outra combinação $\left(-s A_{\mu}^{3(R)}+c B_{\mu}\right)$ realizamos a quebra, agora a mistura é $c \equiv \tilde{g}_{5} \mathrm{e}$ $s \equiv g_{5 R}$ :

$$
\begin{cases}\left.\partial_{5}\left(\tilde{g}_{5} A_{\mu}^{3(R)}+g_{5 R} B_{\mu}\right)\right|_{y=0}=0, & \left.\partial_{5} A_{\mu}^{a(L)}\right|_{y=0}=0 \\ \left.\left(-g_{5 R} A_{\mu}^{3(R)}+\tilde{g}_{5} B_{\mu}\right)\right|_{y=0}=0, & A_{\mu}^{1,2(R)}=0\end{cases}
$$

Vale destacar que a exemplo dos casos anteriores não precisamos impor condições de contorno para os campos $A_{5}^{(L)}, A_{5}^{(R)}$ e $B_{5}$, pois como já discutimos eles são fixados ao impor as condições para os campos vetoriais, bastando trocar Dirichlet por Neumann e vice-versa. Logo não devemos nos preocupar com esses escalares, dado que em pelo menos uma das branas eles satisfazem Dirichlet, impondo por conseguinte que não possuam modo zero. Além disso, como também já vimos, todos os modos com $n>0$ são absorvidos como componente longitudinal dos KK vetoriais. Deste modo podemos focar nossa análise apenas nos bósons vetoriais.

Dado que estamos trabalhando com uma métrica plana, as funções de onda na dimensão extra serão uma combinação de senos e cossenos. Donde se utilizarmos a aproximação $g_{5 R}=g_{5 L}=g_{5}$ e definirmos $A_{\mu}^{L, R \pm} \equiv \frac{1}{\sqrt{2}}\left(A_{\mu}^{1 L, R} \mp i A_{\mu}^{2 L, R}\right)$ podemos expandir os campos vetoriais em modos de Kaluza-Klein satisfazendo as condições de contorno (conforme [11]) como:

$$
\begin{aligned}
B_{\mu}(x, y) & =g_{5} a_{0} \gamma_{\mu}(x)+\tilde{g}_{5} \sum_{n=1}^{\infty} b_{n} \cos \left(M_{n}^{Z}(y-\pi R)\right) Z_{\mu}^{(n)}(x) \\
A_{\mu}^{3(L)}(x, y) & =\tilde{g}_{5} a_{0} \gamma_{\mu}(x)-g_{5} \sum_{n=1}^{\infty} b_{n} \frac{\cos \left(M_{n}^{Z}(y)\right)}{2 \cos \left(M_{n}^{Z} \pi R\right)} Z_{\mu}^{(n)}(x) \\
A_{\mu}^{3(R)}(x, y) & =\tilde{g}_{5} a_{0} \gamma_{\mu}(x)-g_{5} \sum_{n=1}^{\infty} b_{n} \frac{\cos \left(M_{n}^{Z}(y-2 \pi R)\right)}{2 \cos \left(M_{n}^{Z} \pi R\right)} Z_{\mu}^{(n)}(x) \\
A_{\mu}^{ \pm(L)}(x, y) & =\sum_{n=1}^{\infty} c_{n} \cos \left(M_{n}^{W} y\right) W_{\mu}^{ \pm(n)}(x) \\
A_{\mu}^{ \pm(R)}(x, y) & =\sum_{n=1}^{\infty} c_{n} \sin \left(M_{n}^{W} y\right) W_{\mu}^{ \pm(n)}(x)
\end{aligned}
$$

onde o espectro de Kaluza-Klein é formado por um modo não massivo que associamos ao fóton $\gamma(x)$ e por torres de bósons carregados $W_{\mu}^{ \pm(n)}$ e neutros $Z_{\mu}^{(n)}$. O 
espectro de massas dos bósons massivos $W_{\mu}^{ \pm(n)}$ é obtido de

$$
\cos \left(2 M_{W} \pi R\right)=0
$$

logo para os bósons carregados tem-se

$$
M_{n}^{W}=\frac{2 n-1}{4 R}, \quad n \in N^{*} .
$$

Quanto ao espectro de massas dos $Z^{(n)}$ determinamos-no da equação

$$
\tan ^{2}\left(M_{Z} \pi R\right)=1+\frac{\tilde{g}_{5}^{2}}{g_{5}^{2}}
$$

que implica em

$$
\begin{gathered}
M_{n}^{Z}=\left(M_{0}+\frac{n-1}{R}\right), \quad n \in N^{*} \\
M_{n}^{Z^{\prime}}=\left(-M_{0}+\frac{n}{R}\right), \quad n \in N^{*},
\end{gathered}
$$

onde $M_{0}=\frac{1}{\pi R} \arctan \sqrt{1+2 \tilde{g}_{5}^{2} / g_{5}^{2}}$. Como $M_{n}^{Z^{\prime}}>M_{n}^{Z}$ faremos a identificação entre $M_{1}^{Z}$ com a massa do bóson neutro $Z$ do modelo padrão.

Aliando essa identificação ao fato das constantes de acoplamento do MP $g$ e $g^{\prime}$ poderem ser expressas em termos das constantes de acoplamento 5D conforme [21] como

$$
g=\frac{g_{5}}{\sqrt{\pi R}}, \quad g^{\prime}=\frac{\sqrt{2} \tilde{g}_{5}}{\sqrt{\pi R}}
$$

podemos expressar a razão entre as massas dos modos de Kaluza-Klein identificados com os bósons $\mathrm{W}$ e $\mathrm{Z}$ como

$$
\frac{M_{W}^{2}}{M_{Z}^{2}}=\frac{\pi^{2}}{16} \arctan ^{-2} \sqrt{1+\frac{g^{\prime 2}}{g^{2}}} \approx 0.85 \quad \Longrightarrow \quad \rho=\frac{M_{W}^{2}}{M_{Z}^{2} \cos ^{2} \theta_{W}} \approx 1.10 .
$$

Este resultado apesar de apresentar apenas um desvio de $10 \%$ em relação ao obtido pelos testes de precisão do MP (2.89), não pode ser considerado aceitável devido a incrível precisão em que esse parâmetro é medido.

Csáki et al. [22] identificam a origem desse desacordo como proveniente das influências da brana $y=0$, que é a única parte da dimensão extra onde a simetria 
custodial $S U(2)_{L+R}$ está quebrada. Para contornar esse problema eles propõem uma mudança na geometria: da métrica plana até então adotada para a métrica anti-de-Sitter (AdS)

$$
d s^{2}=\left(\frac{R}{z}\right)^{2}\left(\eta_{\mu \nu} d x^{\mu} d x^{\nu}-d z^{2}\right),
$$

onde $\eta_{\mu \nu}=\operatorname{diag}(1,-1,-1,-1)$ e $z$ pertence ao intervalo $\left[R, R^{\prime}\right]$. Nesse novo modelo, Warped Higgsless model, as condições de contorno são as mesmas do caso anterior com métrica plana, contudo a inserção da curvatura diminui a influência da brana onde a simetria custodial não é satisfeita obtendo

$$
\rho=\frac{M_{W}^{2}}{M_{Z}^{2} \cos ^{2} \theta_{W}} \approx 1,
$$

que está de acordo com os resultados experimentais.

Toda teoria que almeja ser uma extensão ao MP tem que necessariamente restaurar a unitariedade até uma escala de energia suficientemente alta (pelo menos mais alta que a escala onde aparece o problema da hierarquia). Dado que no MP essa restauração é efetuada pelo Higgs e que nos modelos por quebra espontânea de simetria por condições de contorno não possuimos tal escalar, devemos analisar qual seria o mecanismo de restauração de unitariedade para esses novos modelos.

\subsection{Unitariedade e os modos de Kaluza-Klein}

Se no MP removermos o Higgs almejando eliminar o problema da hierarquia recaímos em outro grave problema, a saber, a amplitude do espalhamento $W_{L} W_{L} \rightarrow$ $W_{L} W_{L}$ cresceria com $E^{2}$ violando unitariedade por volta de $1.8 \mathrm{TeV}$, conforme (2.110). Um dos papéis desempenhados pelo Higgs no MP é justamente o cancelamento dessa dependência com a energia, deste modo as teorias sem Higgs necessitam de outro dispositivo que providencie esse cancelamento. Tal objetivo pode ser alcançado simplesmente inserindo um novo bóson vetorial massivo com massa próxima a escala $\mathrm{TeV}$, cancelando a divergência quadrática do $W_{L} W_{L} \rightarrow W_{L} W_{L}$, contudo recairíamos no mesmo problema para o espalhamento do novo bóson $W^{\prime}$, ou seja, a amplitude $\mathcal{A}\left(W_{L}^{\prime} W_{L}^{\prime} \rightarrow W_{L}^{\prime} W_{L}^{\prime}\right)$ cresceria com $E^{2}$ como no caso do bóson mais leve. Os modelos sem Higgs tomam partido desse fato para cancelar a divergência através de um procedimento recursivo, este consiste na inserção de uma torre infinita de bósons de Kaluza-Klein, de tal modo que sempre existam 
bósons mais massivos para cancelar a dependência com $E^{2}$ no espalhamento dos mais leves.

Para garantir esse cancelamento algumas relações entre as constantes de acoplamento e as massas dos bósons vetoriais tem que ser satisfeitas, as quais obteremos tendo em vista a referência [20]. Por simplicidade analisaremos o caso de espalhamento elástico longitudinal entre os n-ésimos modos de Kaluza-Klein $n+n \rightarrow n+n$ com índices de gauge $a+b \rightarrow c+d$. Nesse espalhamento existem quatro tipos de diagramas, os dos canais $s, t$ e $u$ (onde há a troca de KK's) e a contribuição proveniente do acoplamento quártico. Estimamos a dependência da amplitude com a energia nesses espalhamentos através das seguintes grandezas cinemáticas no centro de massa

$$
\begin{aligned}
\epsilon_{\mu} & =\left(\frac{|\vec{p}|}{M_{n}}, \frac{E}{M_{n}} \frac{\vec{p}}{|\vec{p}|}\right) \\
p_{\mu}^{\text {in }} & =\left(E, 0,0, \pm \sqrt{E^{2}-M_{n}^{2}}\right) \\
q_{\mu}^{\text {out }} & =\left(E, \pm \sqrt{E^{2}-M_{n}^{2}} \sin \theta, 0, \pm \sqrt{E^{2}-M_{n}^{2}} \cos \theta\right),
\end{aligned}
$$

pois a altas energias temos que $\epsilon \sim E, p_{\mu} \sim E$ e o propagador $\sim E^{-2}$. Então para $E \gg M_{W}$ podemos expandir a amplitude em função da energia como

$$
\mathcal{A}=\mathcal{A}^{(4)} \frac{E^{4}}{M_{n}^{4}}+\mathcal{A}^{(2)} \frac{E^{2}}{M_{n}^{2}}+\mathcal{A}^{(0)}+\mathcal{O}\left(\frac{M_{n}^{2}}{E^{2}}\right) .
$$

No MP o termo $\mathcal{A}^{(4)}$ é nulo e o termo que possui dependência quadrática $\mathcal{A}^{(2)}$ só se anula devido a existência dos diagramas em que há troca do Higgs, como já destacado. Quanto ao caso de uma teoria sem Higgs Chivukula et al. [27] obtém a forma dos termos divergentes, após um cálculo detalhado do espalhamento elástico dos bósons $n$ trocando um bóson $k$. Este obtém a seguinte forma para a divergência com $E^{4}$ :

$\mathcal{A}^{(4)}=i\left(g_{n n n n}^{2}-\sum_{k} g_{n n k}^{2}\right)\left(\epsilon^{a b e} \epsilon^{c d e}\left(3+6 \cos \theta-\cos ^{2} \theta\right)+2\left(3-\cos ^{2} \theta\right) \epsilon^{a c e} \epsilon^{b d e}\right)$.

onde $g_{n n n n}$ e $g_{n n k}$ são as constantes de acoplamento quártico entre quatro bósons $n$ e cúbico entre dois bósons $n$ e um $k$.

Então para $\mathcal{A}^{(4)}$ ser nulo temos que estabelecer a relação que segue entre as 
constantes de acoplamento

$$
g_{n n n n}^{2}=\sum_{k} g_{n n k}^{2}
$$

Tendo em vista que nos modelos sem Higgs oriundos de dimensões extras, as constantes de acoplamento da teoria efetiva estão associadas com as funções de onda $f_{n}(y)$ por $^{4}$

$$
\begin{aligned}
g_{m n k} & =g_{5} \int d y f_{m}(y) f_{n}(y) f_{k}(y) \\
g_{m n k l}^{2} & =g_{5}^{2} \int d y f_{m}(y) f_{n}(y) f_{k}(y) f_{l}(y)
\end{aligned}
$$

deste modo podemos utilizar a relação de completeza

$$
\sum_{k} f_{k}(y) f_{k}(z)=\delta(y-z)
$$

para assegurar que (3.62) seja satisfeita, pois

$$
\begin{aligned}
\sum_{k} g_{n n k}^{2}= & g_{5}^{2} \sum_{k}\left(\int_{0}^{\pi R} d y f_{n}^{2}(y) f_{k}(y)\right)\left(\int_{0}^{\pi R} d z f_{n}^{2}(z) f_{k}(z)\right) \\
& =g_{5}^{2} \int_{0}^{\pi R} d y \int_{0}^{\pi R} d z f_{n}^{2}(y) f_{n}^{2}(z) \delta(y-z) \\
& =g_{5}^{2} \int_{0}^{\pi R} d y f_{n}^{4}(y) \\
& =g_{n n n n}^{2} .
\end{aligned}
$$

Então assumindo que a expressão (3.62) é satisfeita a divergência quadrática com a energia conforme $[20,27]$ adquire a seguinte forma:

$$
\mathcal{A}^{(2)}=\frac{i}{M_{n}^{2}}\left(4 g_{n n n n} M_{n}^{2}-3 \sum_{k} g_{n n k}^{2} M_{k}^{2}\right)\left(\epsilon^{a c e} \epsilon^{b d e}-\sin ^{2} \frac{\theta}{2} \epsilon^{a b e} \epsilon^{c d e}\right)
$$

\footnotetext{
${ }^{4} \mathrm{~A}$ expressão (3.63) é válida apenas quando adotamos a métrica plana. No caso de dimensões extras curvas aparece na integral um fator devido a curvatura. Contudo a relação (3.62) ainda será obtida, pois na relação de completeza também aparece um fator devido a curvatura ratificando o cancelamento da divergência com $E^{4}$.
} 
Assim para haver um cancelamento dessa divergência, devemos exigir que existam infinitos KK satisfazendo uma relação entre as constantes de acoplamento quárticas e cúbicas com as massas dos modos para que o primeiro parênteses se anule

$$
g_{n n n n} M_{n}^{2}=\frac{3}{4} \sum_{k} g_{n n k}^{2} M_{k}^{2} .
$$

Na prova de (3.67) no contexto de dimensões extras, conforme [21], utiliza-se apenas integração por partes e a relação de completeza $(3.64)^{5}$, obtendo que:

$$
\begin{aligned}
\sum_{k} M_{k}^{2} & \left(\int d y f_{n}^{2}(y) f_{k}(y)\right)^{2}=\frac{4}{3} M_{n}^{2} \int d y f_{n}^{4}(y)-\frac{2}{3}\left[f_{n}^{3} f_{n}^{\prime}\right] \\
& -\sum_{k}\left[f_{n}^{2} f_{k}^{\prime}\right] \int d y f_{n}^{2}(y) f_{k}(y)+2 \sum_{k}\left[f_{n} f_{n}^{\prime} f_{k}\right] \int d y f_{n}^{2}(y) f_{k}(y)
\end{aligned}
$$

onde denotamos $[F] \equiv F(\pi R)-F(0)$. Então para condições de contorno arbitrárias (3.32) o cancelamento da divergência com $E^{2}$ não é assegurado. Vemos que para haver esse cancelamento é exigido que as condições de contorno sejam Neumann ou Dirichlet.

As expressões (3.62) e (3.67), em especial, produzem:

$$
\begin{gathered}
g_{W W W W}=g_{W W Z}^{2}+g_{W W \gamma}^{2}+\sum_{k} g_{W W k}^{2} \\
4 g_{W W W W} M_{W}^{2}=3\left[g_{W W Z}^{2} M_{Z}^{2}+\sum_{k} g_{W W k}^{2}\left(M_{k}^{0}\right)^{2}\right]
\end{gathered}
$$

onde $k$ é um bóson de Kaluza-Klein neutro com massa $M_{k}^{0}$.

Se analisarmos de modo análogo o espalhamento $W_{L} Z_{L} \rightarrow W_{L} Z_{L}$ temos que ter satisfeitas novas relações entre as constantes de acoplamento e as massas para eliminar as divergências $E^{2}$ e $E^{4}[29]$, a saber:

$$
g_{W W Z Z}=g_{W W Z}^{2}+\sum_{k} g_{W Z k}^{2}
$$

\footnotetext{
${ }^{5}$ Observe que no modelo padrão o cancelamento de $E^{4}$ é garantido pelas relações $g_{W W W W}^{2}=$ $g^{2}, g_{W W Z}^{2}=g^{2} \cos ^{2} \theta_{W}$ e $g_{W W \gamma}^{2}=g^{2} \sin ^{2} \theta_{W}$, as quais podem ser obtidas diretamente de (2.33), (2.34) e (2.36).
} 


$$
\begin{array}{r}
2\left(g_{W W Z Z}-g_{W W Z}^{2}\right)\left(M_{W}^{2}+M_{Z}^{2}\right)+g_{W W Z}^{2} \frac{M_{Z}^{4}}{M_{W}^{2}} \\
=\sum_{k} g_{W Z k}^{2}\left[3\left(M_{k}^{ \pm}\right)^{2}-\frac{\left(M_{Z}^{2}-M_{W}^{2}\right)^{2}}{\left(M_{k}^{ \pm}\right)^{2}}\right]
\end{array}
$$

onde k é um bóson de Kaluza-Klein carregado de massa $M_{k}^{ \pm}$.

Devemos ressaltar que mesmo tendo satisfeitas as regras de soma obtidas para os casos $W_{L} W_{L} \rightarrow W_{L} W_{L}$ e $W_{L} Z_{L} \rightarrow W_{L} Z_{L}$ cancelando as divergências com $E^{4}$ e $E^{2}$ na amplitude, podemos ainda ter um valor muito alto para $\mathcal{A}^{(0)}$ de modo a ainda termos a unitariedade violada. Essa é uma das facetas da não renormalizabilidade da teoria 5D, que no nosso caso implica em um crescimento linear com a energia da amplitude quando levamos em conta também espalhamentos inelásticos entre os bósons de gauge, conforme [30].

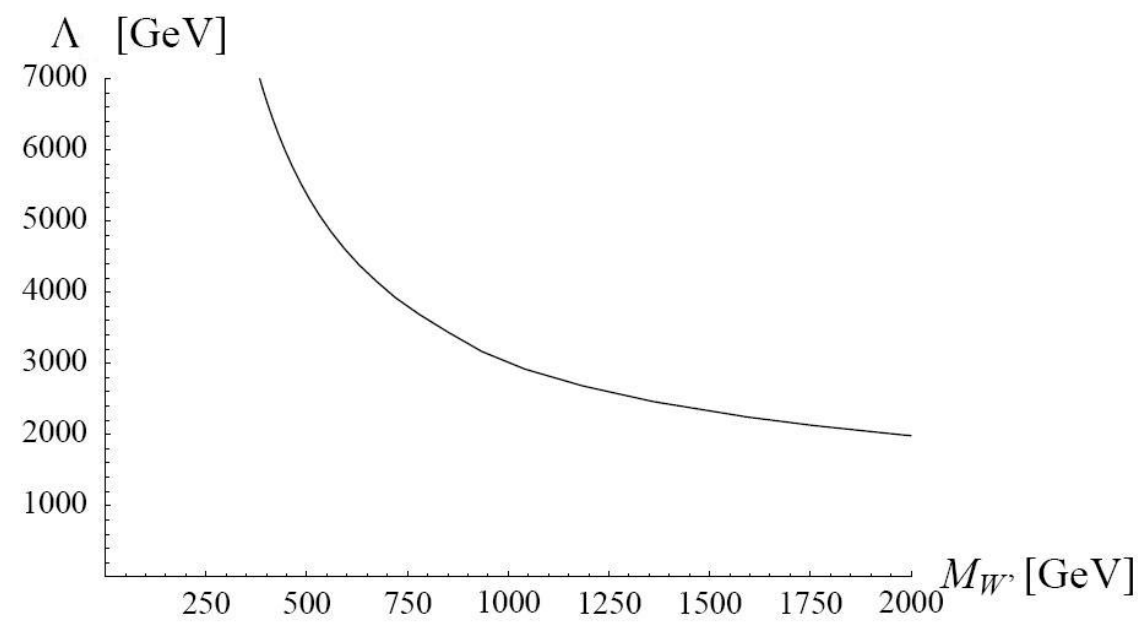

Figura 3.4: Cutoff da teoria sem Higgs com métrica plana em função da massa do primeiro bóson vetorial excitado, ref. [30].

Conforme indicado em [20], a escala de energia em que temos a violação da unitariedade pode ser obtida, a menos de um fator numérico de ordem 1, através da estimativa do cutoff da teoria 5D. Dado que as correções de 1-loop desta são sempre proporcionais a $g_{5}^{2} / 24 \pi^{3}$ e que $\left[g_{5}^{2}\right]=M^{-1}$, então podemos estimar a escala de energia em que o fator adimensional $g_{5}^{2} E / 24 \pi^{3}$ fica da ordem $\mathcal{O}(1)$, ou seja, as contribuições de 1-loop ficam tão relevantes quanto as de ordem de árvore. Assim o cutoff $\Lambda$ é estimado por 


$$
\Lambda=\frac{24 \pi^{3}}{g_{5}^{2}} .
$$

Como em teorias com dimensões extras planas a constante de acoplamento 5D $g_{5}$ pode ser expressa em termos da em $4 \mathrm{D} g$ através de

$$
g_{5}^{2}=\pi R g^{2}
$$

e como $1 / R=M_{W}^{2} / M_{K K^{(1)}}$, onde $M_{W}$ é a massa do $W$ e $M_{1}^{ \pm}$é a massa do primeiro KK acima do $W$. Então temos a seguinte dependência do cutoff com $M_{1}^{ \pm}$

$$
\Lambda=\frac{24 \pi^{2}}{g^{2} R}=\frac{24 \pi^{2}}{g^{2}} \frac{M_{W}^{2}}{M_{1}^{ \pm}},
$$

donde concluímos que quanto menor a massa do primeiro modo de Kaluza-Klein maior será o cutoff da teoria. Papucci [30] analisa essa relação entre $M_{1}^{ \pm}$e o cutoff em teorias sem Higgs com uma dimensão extra plana, como denota a figura (3.4), convém destacar que essa referência leva em conta tanto espalhamentos elásticos quanto inelásticos possuindo assim uma estimativa mais refinada que (3.73). Da figura (3.4) concluímos por exemplo que em teorias sem Higgs com uma dimensão extra plana o cutoff é sempre menor que $5 \mathrm{TeV}$ para $M_{1}^{ \pm}>500 \mathrm{GeV}$. 


\section{Capítulo 4}

\section{Sinais de Produção de Novos Bósons Vetoriais no $\mathrm{LHC}^{1}$}

A restauração da unitariedade no espalhamento $W W \rightarrow W W$ e $W Z \rightarrow W Z$ via inserção de uma torre de bósons vetoriais satisfazendo as regras de soma (3.62) e (3.67) é um mecanismo utilizado por um conjunto bastante extenso de teorias além do MP que não possuem Higgs [24, 28]. Neste conjunto se enquadram os modelos com quebra espontânea de simetria por condições de contorno, apresentados no capítulo anterior.

Apesar do mecanismo de restauração de unitariedade ser o mesmo para toda essa classe de modelos os primeiros indícios concretos a serem vistos no LHC, a saber, as novas ressonâncias vetoriais $Z^{\prime}$ e $W^{\prime}$ possuem massas, larguras e acoplamentos com os férmions altamente dependentes de modelo. Nesse sentido o melhor canal para observação dessas ressonâncias seria via espalhamento de bósons vetoriais, com troca de $Z^{\prime}$ no caso $W^{+} W^{-} \rightarrow W^{+} W^{-}$e de $W^{\prime}$ no caso $Z W^{ \pm} \rightarrow Z W^{ \pm}$, conforme figura (4.1). Todavia no LHC temos apenas a contribuição do $W^{\prime}$ via fusão de bósons vetoriais passível de observação, enquanto as contribuições do $Z^{\prime}$ continuam não observáveis mesmo com o aumento da luminosidade [29].

Visando ampliar essa análise poderíamos considerar a produção desses novos bósons vetoriais através de processos Drell-Yan figura (4.2), onde eles são produzidos pelos seus acoplamentos com quarks leves. Nesse contexto poderiamos estudar processos em que esses novos bósons decaiam em bósons de gauge do MP:

\footnotetext{
${ }^{1}$ Os resultados apresentados neste capítulo foram publicados no artigo [31].
} 


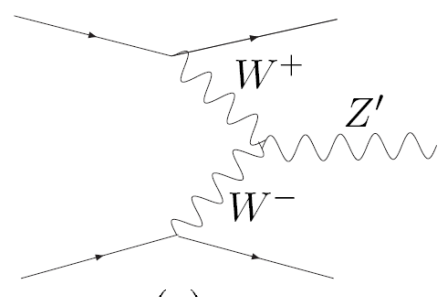

(a)

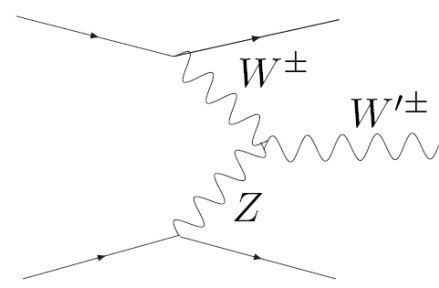

(b)

Figura 4.1: Diagramas de produção de $Z^{\prime}$ e $W^{\prime}$ via fusão de bósons eletrofracos.

$$
\begin{aligned}
& p p \rightarrow Z^{\prime} \rightarrow W^{+} W^{-} \rightarrow l^{+} l^{\prime-} \mathbb{E}_{T} \quad \& \quad l^{ \pm} j j \mathscr{E}_{T} \\
& p p \rightarrow W^{\prime} \rightarrow W^{ \pm} Z \rightarrow l^{ \pm} l^{+} l^{-} \mathbb{E}_{T} ; \quad l^{ \pm} j j \mathscr{E}_{T} \quad \& \quad l^{+} l^{-} j j
\end{aligned}
$$

onde $l$ e $l^{\prime}$ denotam elétrons e muons e $j$ denotam jatos.

Neste capítulo iremos estudar os processos (4.1) e (4.2) por meio de uma análise independente de modelo. Não consideramos o estado final puramente hadrônico dado que este possui um alto background de QCD, deste modo focamos nossa análise nos estados finais leptônicos e semi-leptônicos. Cabe destacar que a análise dos processos puramente leptônicos foi efetuada pelos demais colaboradores em [31], contudo apresento seus resultados com o intuito de ampliar nossa possibilidade de discussão.

Efetuamos aqui uma análise independente de modelo expressando os resultados em termos das massas, larguras e dos acoplamentos relevantes dos novos bósons de spin-1. Deste modo complementamos a análise já realizada para o $W^{\prime}$ no contexto de espalhamento de bósons vetoriais e evidenciamos a possibilidade de observação do $Z^{\prime}$ nos processos que analisamos, dado que os processos associados a espalhamento de bósons vetoriais neste caso não são passíveis de observação no LHC.

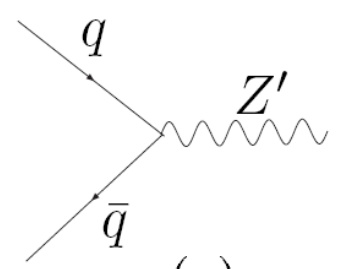

(a)

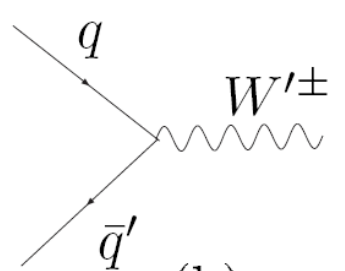

(b)

Figura 4.2: Diagramas de produção de $Z^{\prime}$ e $W^{\prime}$ via processos Drell Yan. 


\subsection{Estrutura da análise}

Visando efetuar uma análise independente de modelo dos processos (4.1) e (4.2) devemos impor apenas vínculos de consistência aos parâmetros dependentes de modelo, a saber, massas e larguras dos novos bósons vetoriais além dos seus acoplamentos com os quarks e bósons de gauge do MP.

Com isso em vista assumimos que os acoplamentos do $Z^{\prime}$ e $W^{\prime}$ com quarks leves e bósons vetoriais tem a mesma estrutura de Lorentz que os dos bósons do MP. Os Higgsless models possuem tal propriedade diferindo apenas em intensidade com o MP.

Como vimos esses modelos satisfazem determinadas regras de soma assegurando a unitariedade. No caso do espalhamento $W^{+} W^{-} \rightarrow W^{+} W^{-}$se tomarmos que sua regra de soma estiver saturada pelo primeiro bóson de Kaluza-Klein obtemos de (3.69) que

$$
g_{Z^{\prime} W W \max }=g_{Z W W} \frac{M_{Z}}{\sqrt{3} M_{Z^{\prime}}}
$$

para uma dada massa $M_{Z^{\prime}}$. Analogamente o limite de saturação do espalhamento $W^{ \pm} Z \rightarrow W^{ \pm} Z$ pode ser obtido de (3.70)

$$
g_{W^{\prime} W Z \max }=g_{W W Z} \frac{M_{Z}^{2}}{\sqrt{3} M_{W^{\prime}} M_{W}} .
$$

Esses novos parâmetros $\left(g_{Z^{\prime} W W \max }, g_{W^{\prime} W Z \max }\right)$ serão utilizados nesse trabalho apenas como fator de normalização dos acoplamentos $g_{Z^{\prime} W W}$ e $g_{W^{\prime} W Z}$. Além disso vamos tratar as larguras $\Gamma_{Z^{\prime}}$ e $\Gamma_{W^{\prime}}$ como parâmetros, pois os KK podem ter acoplamentos com outras partículas que não estamos considerando, a exemplo de quarks mais pesados $b$ e $t$ alterando o valor da largura total.

Deste modo a seção de choque dos processos (4.1) e (4.2) tem a seguinte forma geral:

$\sigma_{t o t}=\sigma_{M P}+\left(\frac{g_{V^{\prime} q \bar{q}}}{g_{V q \bar{q}}} \frac{g_{V^{\prime} W V}}{g_{V^{\prime} W V \max }}\right) \sigma_{i n t}\left(M_{V^{\prime}}, \Gamma_{V^{\prime}}\right)+\left(\frac{g_{V^{\prime} q \bar{q}}}{g_{V q \bar{q}}} \frac{g_{V^{\prime} W V}}{g_{V^{\prime} W V \max }}\right)^{2} \sigma_{V^{\prime}}\left(M_{V^{\prime}}, \Gamma_{V^{\prime}}\right)$

sendo que para os processos (4.1) $V^{\prime}=Z^{\prime}, g_{V^{\prime} W V} \equiv g_{Z^{\prime} W W}$ e $g_{V q \bar{q}} \equiv g_{Z q \bar{q}}=$ $g / \cos \theta_{W}$. Para os processos (4.2) $V^{\prime}=W^{\prime}, g_{V^{\prime} W V} \equiv g_{W^{\prime} W Z}$ e $g_{V q \bar{q}} \equiv g_{W q \bar{q}}=g / \sqrt{2}$ 2 .

\footnotetext{
${ }^{2}$ Continuamos denotando $g$, como nos capítulos anteriores, como constante de acoplamento
} 
Conforme denota a fórmula (4.5) esta análise possui três parâmetros livres: a massa do novo bóson vetorial $M_{V^{\prime}}$, a largura $\Gamma_{V^{\prime}}$ e o produto das constantes de acoplamento do bóson vetorial $V^{\prime}$ com quarks leves e com os bósons de gauge do MP. Esses parâmetros possuem apenas um vínculo, dado que os novos bósons podem se acoplar com outras partículas além de quarks leves e os bósons de gauge do MP temos:

$$
\begin{aligned}
& \Gamma_{Z^{\prime}} \geq \sum_{q=u, d} \Gamma\left(Z^{\prime} \rightarrow q \bar{q}\right)+\Gamma\left(Z^{\prime} \rightarrow W^{+} W^{-}\right) \\
& \Gamma_{W^{\prime}} \geq \Gamma\left(W^{\prime+} \rightarrow u \bar{d}\right)+\Gamma\left(W^{\prime+} \rightarrow W^{+} Z\right)
\end{aligned}
$$

tendo em vista os seguintes resultados para as larguras parciais (em $\mathrm{GeV})$ :

$$
\begin{aligned}
\Gamma\left(Z^{\prime} \rightarrow u \bar{u}\right) & =0.3\left(\frac{M_{Z^{\prime}}}{M_{Z}}\right)\left(\frac{g_{Z^{\prime} q \bar{q}}}{g_{Z q \bar{q}}}\right)^{2} \\
\Gamma\left(Z^{\prime} \rightarrow d \bar{d}\right) & =0.38\left(\frac{M_{Z^{\prime}}}{M_{Z}}\right)\left(\frac{g_{Z^{\prime} q \bar{q}}}{g_{Z q \bar{q}}}\right)^{2} \\
\Gamma\left(Z^{\prime} \rightarrow W^{+} W^{-}\right) & =0.028\left(\frac{M_{Z^{\prime}}}{M_{Z}}\right)^{3}\left(\frac{g_{Z^{\prime} W W}}{g_{Z^{\prime} W W \max }}\right)^{2} \\
\Gamma\left(W^{\prime+} \rightarrow q^{\prime} \bar{q}\right) & =0.68\left(\frac{M_{W^{\prime}}}{M_{W}}\right)\left(\frac{g_{W^{\prime} q \bar{q}}}{g_{W q \bar{q}}}\right)^{2} \\
\Gamma\left(W^{\prime+} \rightarrow W^{+} Z\right) & =0.019\left(\frac{M_{W^{\prime}}}{M_{W}}\right)^{3}\left(\frac{g_{W^{\prime} W Z}}{g_{W^{\prime} W Z \max }}\right)^{2}
\end{aligned}
$$

obtemos os seguintes vínculos para as larguras totais $\Gamma_{V^{\prime}}(\mathrm{em} \mathrm{GeV})$ :

$$
\begin{gathered}
\Gamma_{Z^{\prime}}>0.27\left(\frac{g_{Z^{\prime} q \bar{q}}}{g_{Z q \bar{q}}}\right)\left(\frac{g_{Z^{\prime} W W}}{g_{Z W W \max }}\right)\left(\frac{M_{Z^{\prime}}}{M_{Z}}\right)^{2} \\
\Gamma_{W^{\prime}}>0.40\left(\frac{g_{W^{\prime} q \bar{q}}}{g_{W q \bar{q}}}\right)\left(\frac{g_{W^{\prime} W Z}}{g_{W^{\prime} W Z \max }}\right)\left(\frac{M_{W^{\prime}}}{M_{W}}\right)^{2} .
\end{gathered}
$$

Na obtenção da seção de choque efetuamos um cálculo a nível de pártons utilizando o programa MadGraph Stand Alone ${ }^{3}$ [33] para gerar as amplitudes dos subprocessos. Para efetuar o cálculo da seção de choque foi criado um programa do grupo $S U(2)_{L}$.

${ }^{3}$ Adicionando neste os novos estados e interações. 
que realizava a integração no espaço de fase via método de Monte Carlo utilizando as amplitudes dos subprocessos $|\mathcal{M}|^{2}$ (subprocesso) gerada pelo MadGraph:

$\sigma=\int d x_{1} d x_{2} \sum_{i, j} f_{i}^{(a)}\left(x_{1}, \mu_{F}^{2}\right) f_{j}^{(b)}\left(x_{2}, \mu_{F}^{2}\right) \frac{1}{2 \hat{s}} \int d \Phi_{n} \Theta($ cortes $)|\mathcal{M}|^{2}$ (subprocesso)

onde $f_{i}^{(a)}\left(x_{i}, \mu_{F}^{2}\right)$ são as funções de distribuição partônicas, que fornecem a probabilidade de encontrar o parton $i$ dentro do hadron $a$ com fração de momento $x_{i}$, em um processo que possui uma escala típica de energia (escala de fatorização) $\mu_{F}$. A energia do centro de massa $\sqrt{s}$ se relaciona com $\hat{s}$ por $\hat{s}=x_{1} x_{2} s$. Por fim, temos que o espaço de fase é dado por

$$
d \Phi_{n}\left(P ; p_{1} \ldots p_{n}\right)=\prod_{i=1}^{n}\left(\frac{d^{3} p_{i}}{(2 \pi)^{3} 2 E_{i}}\right)(2 \pi)^{4} \delta^{4}\left(P-\sum_{i} p_{i}\right) .
$$

A seção de choque em geral não é avaliada em todo o espaço de fase, mas apenas na região onde as partículas do estado final são visíveis no detector. Na verdade adiciona-se mais cortes no espaço de fase a esses cortes básicos visando extrair algum sinal específico do background total.

Como antecipado nesta seção, nossas simulações foram realizadas a nível de pártons, ou seja, interpretamos quarks e glúons como jatos. Almejando efetuar uma análise mais próxima da realidade simulamos as resoluções experimentais inserindo um erro Gaussiano na energia (Gaussian smearing), mas não na direção, conforme tabela (4.1), onde $\oplus$ indica soma em quadratura e $\eta$ a pseudo-rapidez. ${ }^{4}$

\begin{tabular}{|c|c|c|c|}
\hline & léptons & jatos com $\left|\eta_{j}\right| \leq 3$ & jatos com $\left|\eta_{j}\right|>3$ \\
\hline$\frac{\Delta E}{E}$ & $\frac{0.1}{\sqrt{E}} \oplus 0.01$ & $\frac{0.5}{\sqrt{E}} \oplus 0.03$ & $\frac{1}{\sqrt{E}} \oplus 0.07$ \\
\hline
\end{tabular}

Tabela 4.1: Smearing na energia $E$ (em GeV) com erro Gaussiano.

Ainda visando uma simulação mais próxima ao obtido experimentalmente, para o momento transversal do neutrino atribuimos um valor distinto ao gerado pelo Monte Carlo. O momento transversal desta partícula será identificado como a soma vetorial dos momentos das partículas do estado final após o smearing, ou seja, será o momento transversal "faltante" $\not p_{T}{ }^{5}$, analogamente ao que é feito ex-

\footnotetext{
${ }^{4}$ A pseudo-rapidez $\eta$ é fornecida em termos do ângulo polar $\theta$ por: $\eta=-\ln \tan \left(\frac{\theta}{2}\right)$.

${ }^{5} p_{T}$ é o momento transversal definido por $p_{T}=\sqrt{p_{x}^{2}+p_{y}^{2}}$, onde o eixo z é o eixo de deslocamento do feixe.
} 
perimentalmente.

Além disso foi utilizado uma eficiência de 0.9 na detecção de léptons. Nos cálculos utilizamos as distribuições partônicas CTEQ6L que conforme indicado em [34] se ajustam melhor a integrações via método de Monte Carlo. Tomamos como escala de renormalização e fatorização $\mu_{F}=\mu_{R}=\sqrt{\left(p_{T}^{(l+) 2}+p_{T}^{(l-) 2}\right) / 2}$ para estados finais puramente leptônicos e para canais semi-leptônicos utilizamos $\mu_{F}=\mu_{R}=\sqrt{\left(p_{T}^{\left(j_{1}\right) 2}+p_{T}^{\left(j_{2}\right) 2}\right) / 2}$. Por fim adotamos uma energia do centro de massa de $\sqrt{s}=14 \mathrm{TeV}$.

\subsection{Análise dos cortes}

Nesta seção analisaremos os processos (4.1) e (4.2) visando extrair os sinais $Z^{\prime}$ e $W^{\prime}$ do background. Para tal discutiremos quais são os backgrounds mais importantes e os cortes mais eficientes na sua supressão.

\subsection{1 $\quad p p \rightarrow Z^{\prime} \rightarrow W^{+} W^{-} \rightarrow l^{ \pm} j j E_{T}$}

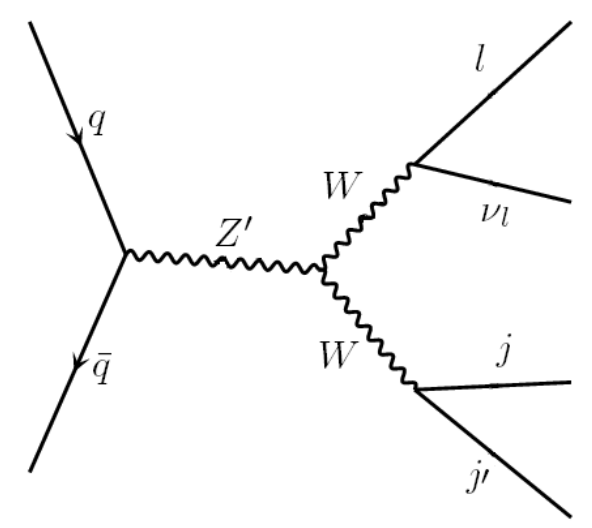

Figura 4.3: Diagrama do sinal $p p \rightarrow Z^{\prime} \rightarrow W^{+} W^{-} \rightarrow l^{ \pm} j j \mathbb{E}_{T}, \operatorname{com} l^{ \pm}=e^{ \pm}, \mu^{ \pm}$.

Vamos iniciar a análise discutindo a observabilidade do $Z^{\prime}$ no canal onde um dos $W$ decai em léptons $W \rightarrow l \nu_{l}(l=e, \mu)$ e o outro em jatos $W \rightarrow j j^{\prime}$. Apesar de possuir um background mais alto que o caso puramente leptônico, esse canal possui a vantagem de ter um branching ratio alto, $B R(W W) \approx 2 / 9 \times 6 / 9 \times 2=8 / 27$, onde o fator 2 provém da inclusão do $l^{+}$e do $l^{-}$.

Esse como todos os demais sinais estudados neste capítulo possuem o novo bóson vetorial no canal $s$, como denota a figura (4.3). Isso nos possibilita ver 
o sinal de nova física como uma ressonância, desde que consigamos reconstruir a massa invariante do $Z^{\prime}$ a partir do estado final e tenhamos suprimido o background de modo a que o sinal apareça claramente.

O background do processo $p p \rightarrow Z^{\prime} \rightarrow W^{+} W^{-} \rightarrow l^{ \pm} j j \mathbb{E}_{T}$ foi calculado em ordem $\mathcal{O}\left(\alpha_{E M}^{4}\right)$ e $\mathcal{O}\left(\alpha_{E M}^{2} \alpha_{s}^{2}\right)$ de onde destacamos três contribuições dominantes:

1. O background proveniente da produção de $W j j$ com o $W$ decaindo leptonicamente:

$$
p p \rightarrow W^{ \pm} j j \rightarrow l^{ \pm} \nu_{l} j j
$$

2. O de produção do $Z j j$ com o $Z$ decaindo em léptons com um deles não sendo detectado:

$$
p p \rightarrow Z j j \rightarrow l^{+} l^{-} j j
$$

3. Por fim o background de produção de $t \bar{t}$ que é praticamente onipresente no LHC, onde no nosso caso dois dos jatos do estado final não são vistos:

$$
p p \rightarrow t \bar{t} \rightarrow b l^{ \pm} \nu_{l} \bar{b} j j
$$

neste caso geramos o $t \bar{t}$ em ordem $\mathcal{O}\left(\alpha_{s}^{2}\right)$ e decaimos os tops para o estado final.

Quanto ao $t \bar{t}$ iremos requerer o veto dos jatos adicionais com

$$
\left|\eta_{j}\right|<3 \text { e } p_{T}^{j}>20 \mathrm{GeV}
$$

garantindo que esses jatos não sejam vistos. Dado que no LHC no período de alta luminosidade possuiremos aproximadamente 25 interações por bunch crossing, então ao considerarmos a probabilidade de um evento passar por esses cortes devemos levar em conta ainda a probabilidade de detecção de jatos extras devido ao pile-up. Levando em conta esses efeitos temos que a probabilidade de um evento passar pelo veto de jatos adicionais com amplitudes proporcionais a acoplamentos puramente eletromagnéticos $\alpha_{E M}$ e com acoplamentos de QCD $\alpha_{s}$, conforme ref. [31], dadas por:

$$
P^{E W}=0.56, \quad P^{Q C D}=0.23
$$

$\mathrm{Na}$ análise dos melhores cortes para supressão desses backgrounds devemos partir de alguns cortes fundamentais, os quais estão associados as limitações dos 
detectores:

$$
\left|\eta_{l}\right|<2.5 ; \quad\left|\eta_{j}\right|<3 ; \quad \Delta R_{j j(j l)}>0.4
$$

onde $\Delta R \equiv \sqrt{(\Delta \eta)^{2}+(\Delta \phi)^{2}}$ ( $\phi$ é o ângulo azimutal) define o tamanho do cone de separação entre as partículas do estado final.

A estratégia básica de redução dos backgrounds é buscar características peculiares ao sinal das quais o background não compartilhe. Como o $Z^{\prime}$ possui uma massa muito mais alta que as partículas do MP é esperado que o sinal produza jatos e léptons com alto momento transversal. Com isso em vista realizamos um estudo das distribuições do momento transversal do jato menos energético $p_{T}^{j \text { min }}$, do jato mais energético $p_{T}^{j \max }$, do lépton carregado $p_{T}^{l}$ e da energia faltante $\mathbb{E}_{T}$ para o background total e sinal $p p \rightarrow Z^{\prime} \rightarrow l^{ \pm} j j \mathbb{E}_{T}$, como ilustra a figura (4.4) no caso especial $M_{Z^{\prime}}=0.5 \mathrm{TeV}$.

Assim a razão sinal background pode ser aumentada requerendo jatos e léptons mais energéticos. Para tal efetuamos os seguintes cortes:

$$
\begin{array}{ll}
p_{T}^{j}>p_{T}^{j \min }, & \left.p_{T}^{j}\right|_{\max }>p_{T}^{j \max } \\
p_{T}^{l}>p_{T}^{l \min }, & \mathbb{E}_{T}>\mathbb{E}_{T}^{\min }
\end{array}
$$

onde denotamos por $p_{\text {Tmax }}^{j}$ o momento tranversal do jato mais energético. $\mathrm{O}$ melhor conjunto de cortes depende da massa do $Z^{\prime}$ como expresso na tabela (4.2).

\begin{tabular}{|c|c|c|c|}
\hline$M_{Z^{\prime}}(\mathrm{TeV})$ & 0.5 & 1.0 & 1.5 \\
\hline \hline$p_{T}^{l \min }(\mathrm{GeV})$ & 75 & 100 & 100 \\
$p_{T}^{j \min }(\mathrm{GeV})$ & 60 & 75 & 100 \\
$p_{T}^{j \max }(\mathrm{GeV})$ & 110 & 75 & 100 \\
$\mathbb{E}_{T}^{\min }(\mathrm{GeV})$ & 50 & 75 & 75 \\
$\delta(\mathrm{GeV})$ & 50 & 100 & 200 \\
\hline
\end{tabular}

Tabela 4.2: Cortes para o $p p \rightarrow Z^{\prime} \rightarrow W^{+} W^{-} \rightarrow l^{ \pm} j j \mathbb{E}_{T}$ em função da massa do $Z^{\prime}$.

Outra característica peculiar ao sinal que podemos utilizar é a identificação dos dois jatos como provenientes do decaimento do $W$, requerendo que a massa invariante deles $M_{j j}=\left(p_{j_{1}}+p_{j_{2}}\right)^{2}$ seja compatível com a massa do $W$ :

$$
\left|M_{j j}-M_{W}\right|<10 \mathrm{GeV}
$$



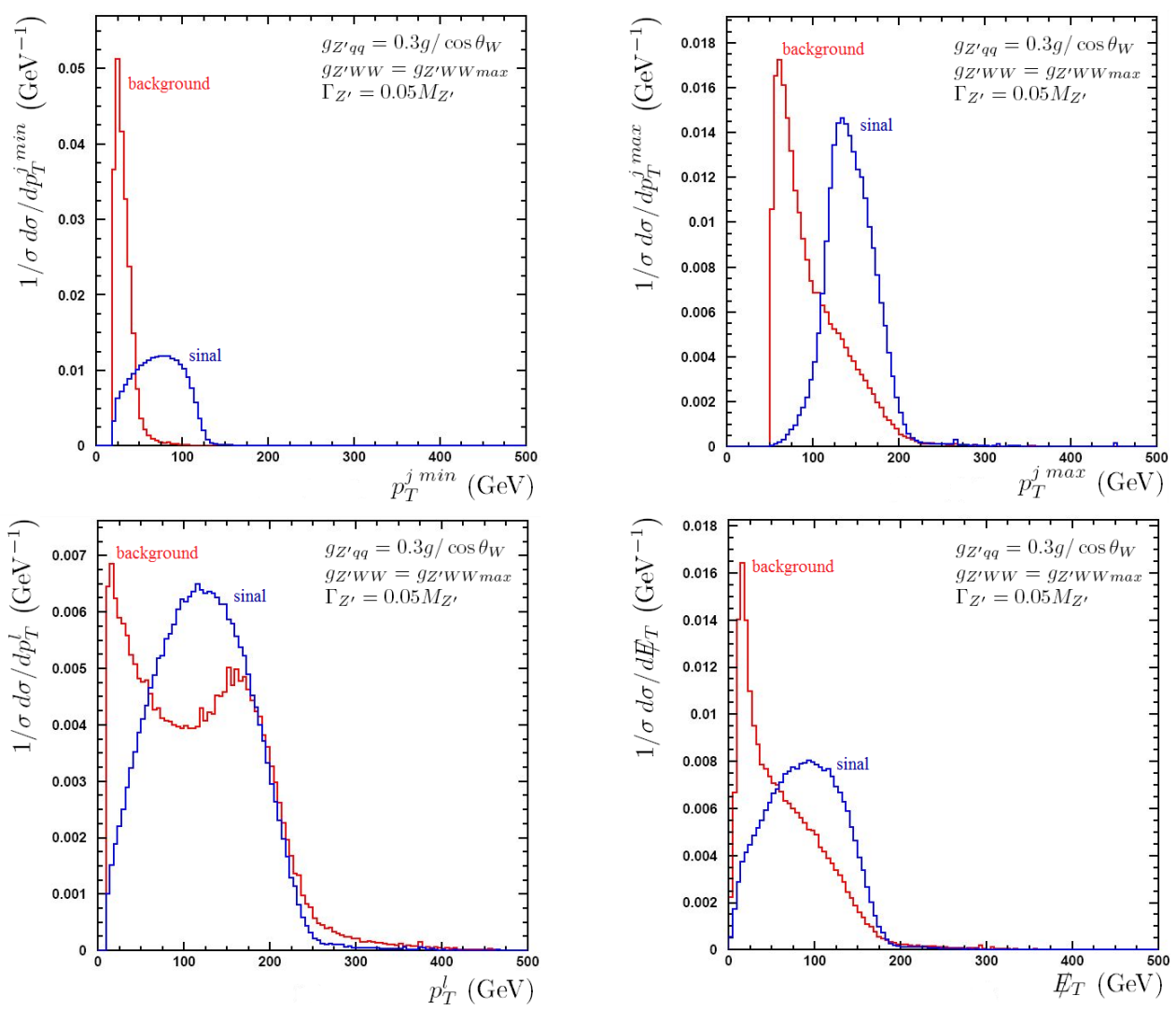

Figura 4.4: Distribuições do momento transversal do jato menos energético $p_{T}^{j \min }$, do jato mais energético $p_{T}^{j \max }$, do lépton carregado $p_{T}^{l}$ e da energia faltante $\mathbb{E}_{T}$ para o background total e sinal $p p \rightarrow Z^{\prime} \rightarrow l^{ \pm} j j \mathbb{E}_{T}$. O histograma em vermelho corresponde ao background e o azul ao sinal com $M_{Z^{\prime}}=0.5 \mathrm{TeV}$ e largura $\Gamma_{Z^{\prime}}=$ $0.05 M_{Z^{\prime}}$. O histograma $p_{T}^{j \text { min }}$ possui apenas os cortes básicos (4.18), o histograma $p_{T}^{j \max }$ possui além dos cortes básicos o corte em $p_{T}^{j \min }$ tabela (4.2), o $p_{T}^{l}$ possui todos os cortes anteriores e o em $p_{T}^{j \max }$, por fim o $\mathbb{E}_{T}$ possui todos os anteriores e o em $p_{T}^{l}$.

Podemos ir ainda mais longe, dado que o estado final possui apenas um único neutrino, isso permite-nos a reconstrução do seu momento impondo conservação do momento transversal e requerendo que a massa invariante do par neutrino- $l^{ \pm}$ igual a $M_{W}, M_{W}^{2}=\left(p_{l}+p_{\nu_{l}}\right)^{2}$, para determinarmos o momento longitudinal do neutrino, conforme ref. [32]:

$$
p_{L}^{\nu}=\frac{1}{2 p_{T}^{l 2}}\left\{\left[M_{W}^{2}+2\left(\vec{p}_{T}^{l} \cdot \vec{p}_{T}\right)\right] p_{L}^{l} \pm \sqrt{\left[M_{W}^{2}+2\left(\vec{p}_{T}^{l} \cdot \vec{p}_{T}\right)\right]\left|\vec{p}^{l}\right|^{2}-4\left(p_{T}^{l} E^{l} \mathbb{E}_{T}\right)^{2}}\right\}
$$

note que o momento longitudinal possui duas raízes. Apesar disso esse procedi- 
mento nos abriu a possibilidade de reconstrução da ressonância do $Z^{\prime}$ para isolarmos ainda mais o sinal do background. Na figura (4.5) temos a massa reconstruída do $W W, M_{r e c}^{\text {min } 2}=\left(p_{\nu_{l}}^{\text {min }}+p_{l}+p_{j_{1}}+p_{j_{2}}\right)^{2}$, com a solução de menor valor para o momento longitudinal do neutrino após os cortes (4.18) - (4.20). Nesta figura observa-se a maior amplitude do sinal em relação ao background na região próxima a $M_{Z^{\prime}}$.

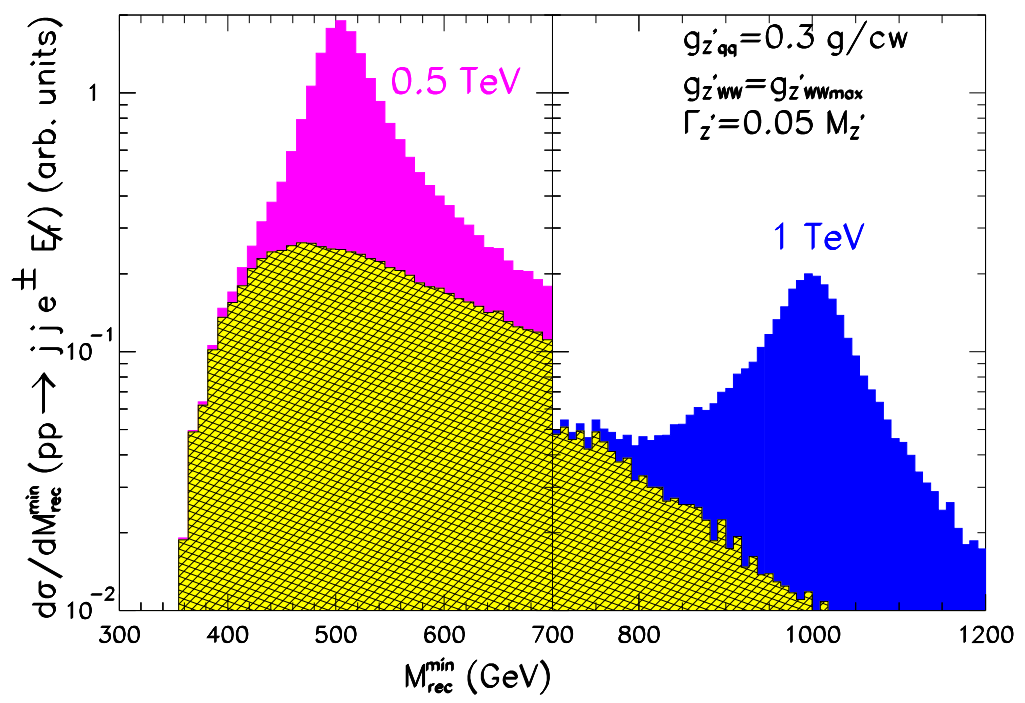

Figura 4.5: Distribuição para a massa invariante reconstruída para o processo $p p \rightarrow j j l^{ \pm} \mathbb{E}_{T}$ utilizando o menor valor de momento longitudinal para o neutrino. Com sinal $M_{Z^{\prime}}=0.5 \mathrm{TeV}$ no painel da esquerda e $1.0 \mathrm{TeV}$ no da direita sobre os respectivos backgrounds hachurados, após os cortes (4.18) - (4.20).

Para as larguras consideradas na análise a ressonância do $Z^{\prime}$ pode ser observada exigindo que $M_{r e c}^{\text {min }}$ seja compatível com $M_{Z^{\prime}}$ dentro de um intervalo $\delta$ :

$$
\left|M_{r e c}^{\min }-M_{Z^{\prime}}\right|<\delta
$$

onde esse intervalo foi escolhido de modo a garantir que a maior parte do sinal esteja nele. Os valores adotados para $\delta$ constam na tabela (4.2).

A tabela (4.3) contém a seção de choque para as diferentes contribuições do background do MP após os cortes (4.18) - (4.22), além da seção de choque para o sinal normalizado como em (4.5) para $M_{Z^{\prime}}=0.5,1.0$ e $1.5 \mathrm{TeV}$ para o valor ilustrativo $\Gamma_{Z^{\prime}}=0.05 M_{Z^{\prime}}$. Cabe destacar que após aplicarmos os cortes a parte de interferência $\sigma_{\text {int }}$ fica negligível para os valores utilizados de massas e larguras 


\begin{tabular}{|c|c|c|c|c|}
\hline$M_{Z^{\prime}}(\mathrm{TeV})$ & $\sigma_{M P}^{E W}(\mathrm{fb})$ & $\sigma_{M P}^{Q C D}(\mathrm{fb})$ & $\sigma_{M P}^{t \bar{t}}(\mathrm{fb})$ & $\sigma_{Z^{\prime}}(\mathrm{fb})$ \\
\hline \hline 0.5 & 17.6 & 208 & 19.2 & 1232 \\
1.0 & 5.6 & 46 & 5.6 & 270 \\
1.5 & 1.5 & 12 & 1.8 & 9.5 \\
\hline
\end{tabular}

Tabela 4.3: Seção de choque para o sinal $Z^{\prime}$ e background em fb para o estado final $j j l^{ \pm} \mathbb{E}_{T}(l=e, \mu)$ após os cortes (4.18) - (4.22). Nesses resultados ainda não foram incluídos as eficiências experimentais de reconstrução de jatos e léptons. Para o sinal apresentamos os resultados para o valor ilustrativo $\Gamma_{Z^{\prime}}=0.05 M_{Z^{\prime}}$.

para o $Z^{\prime}$.

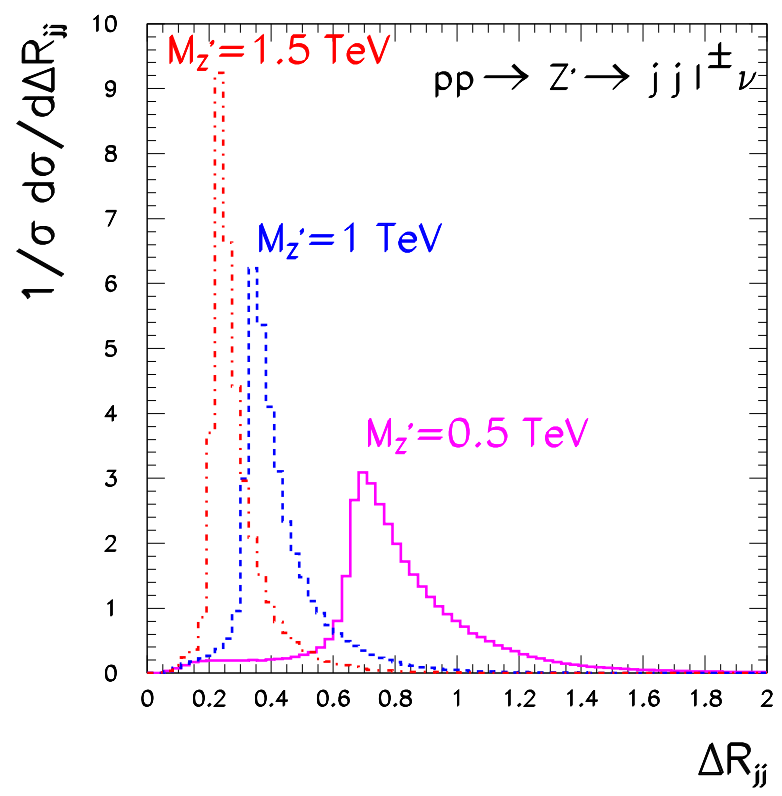

Figura 4.6: Distribuições para $\Delta R_{j j}$ do sinal com estado final $l^{ \pm} j j \mathbb{E}_{T}$ para diferentes valores de $M_{Z^{\prime}}$ no caso ilustrativo $\Gamma_{Z^{\prime}}=0.05 M_{Z^{\prime}}$.

Nessa tabela observa-se uma drástica redução para o sinal quando $M_{Z^{\prime}} \geq 1$ $\mathrm{TeV}$. Isso se deve aos $W^{\prime} s$ serem altamente energéticos nessa região de parâmetros, implicando que seus produtos sejam bem colimados não passando nos cortes básicos (4.18). Podemos constatar tal fato na figura (4.6) onde mostramos as distribuições de $\Delta R_{j j}$ para o sinal com diferentes valores de $M_{Z^{\prime}}$ tendo apenas os cortes em rapidez (4.18) aplicados. Observamos que quanto maior a massa do $Z^{\prime}$ mais colimados saem os jatos implicando no caso $M_{Z^{\prime}}=1.5 \mathrm{TeV}$ que a maior parte do sinal seja perdida quando aplicamos o corte de isolamento.

Com $\Delta R_{j j}>0.4$ implica que o algorítimo de reconstrução dos jatos decaindo 
de um $W$ muito energético iria reconstruir os dois jatos como um só. Deste modo poderiamos tentar evitar a supressão do sinal levando em conta também o estado final $l^{ \pm} j \mathbb{E}_{T}$, como discutido em Agashe et al. [35]. Todavia essa análise exige um estudo muito mais detalhado dos detalhes do detector, que está além do escopo deste trabalho. Nos ateremos apenas ao estado final $l^{ \pm} j j \mathbb{E}_{T}$, mas não podemos perder de vista que os resultados que obtemos para $M_{Z^{\prime}}$ de grande magnitude podem ainda ser melhorados.

\subsection{2 $\quad p p \rightarrow Z^{\prime} \rightarrow W^{+} W^{-} \rightarrow l^{+} l^{\prime-} \mathbb{E}_{T}$}

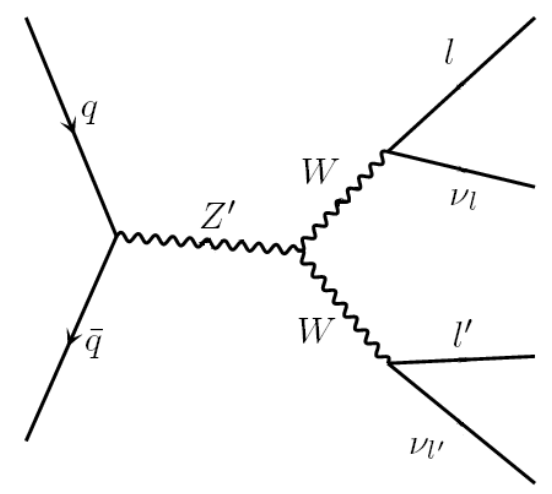

Figura 4.7: Diagrama do sinal $p p \rightarrow Z^{\prime} \rightarrow W^{+} W^{-} \rightarrow l^{ \pm} l^{\prime \pm} \mathbb{E}_{T}$, com $l^{ \pm}, l^{\prime \pm}=$ $e^{ \pm}, \mu^{ \pm}$

Podemos observar o $Z^{\prime}$ também pelo canal puramente leptônico $p p \rightarrow Z^{\prime} \rightarrow$ $W^{+} W^{-} \rightarrow l^{+} l^{\prime-} \notin_{T}$ figura (4.7), conforme referência [31]. Este canal apesar de ser mais limpo que o semi-leptônico do ponto de vista observacional, apresenta dois problemas: o primeiro refere-se a impossibilidade de reconstrução da massa do $Z^{\prime}$ a partir do estado final dado que este possui dois neutrinos, a outra deficiência desse provém do seu baixo branching ratio $B R(W W) \approx(2 / 9)^{2}=4 / 81$.

Os backgrounds do MP dominantes na produção de léptons de diferentes sabores provém da produção de pares $W^{+} W^{-}$e do $t \bar{t}$ com os tops decaindo semileptonicamente. Vamos partir dos cortes básicos em $\eta$ e $\Delta R$ para assegurar detecção e isolamento das partículas do estado final, suplementado pelo corte em $p_{T}^{l}$ dado que como no caso anterior devido ao $Z^{\prime}$ ter uma massa muito alta implica em um sinal com léptons muito energéticos:

$$
\left|\eta_{l}\right|<2.5, \quad \Delta R_{l l}>0.4, \quad \text { e } \quad p_{T}^{l}>50 \mathrm{GeV}
$$


Apesar de não ser possível reconstruir a massa invariante do sinal devido a presença de dois neutrinos no estado final, podemos caracterizar parcialmente o sinal utilizando a variável $M_{T}^{W W}$ definida por:

$$
M_{T}^{W W}=\left[\left(\sqrt{\left(p_{T}^{l^{+} l^{\prime-}}\right)^{2}+m_{l^{+} l^{\prime-}}^{2}}+\sqrt{\not p_{T}^{2}+m_{l^{+} l^{-}}^{2}}\right)^{2}-\left(\vec{p}_{T}^{+l^{\prime-}}+\vec{p}_{T}\right)^{2}\right]^{1 / 2}
$$

onde $\ddot{p}_{T}$ é o momento transversal faltante, $p_{T}^{l^{+} l^{\prime-}}$ é o momento transversal do par $l^{+} l^{-}$e $m_{l+l^{\prime}}$ é a massa invariante do par $l^{+} l^{\prime-}$. Observa-se que a razão sinal background pode ser ampliada se aplicarmos o seguinte corte:

$$
M_{T}^{W W}>\frac{M_{Z^{\prime}}}{2}
$$

Como discutido na seção anterior o background $t \bar{t}$ pode ser reduzido aplicando o veto de jatos adicionais utilizando (4.16) que corresponde as probabilidade dos eventos passar pelo veto (4.17).

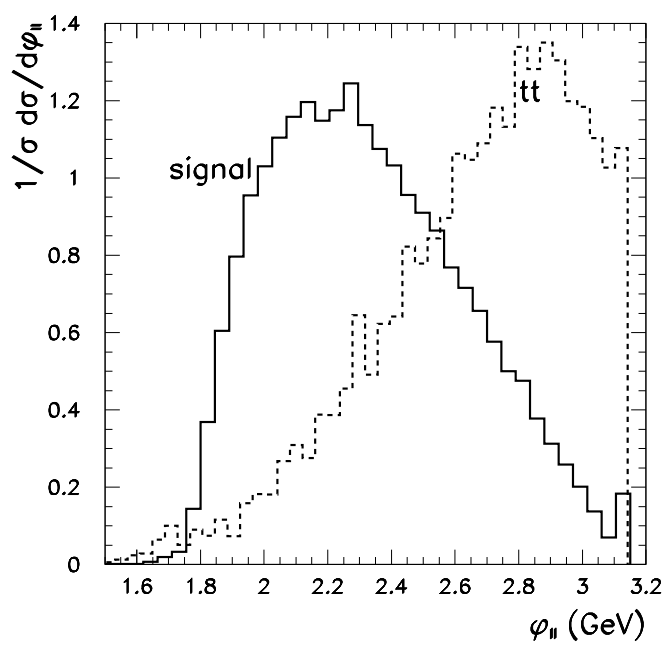

Figura 4.8: Distribuição angular azimutal da separação entre os léptons carregados do estado final $l^{+} l^{\prime-} \mathbb{E}_{T}$. A linha sólida representa a distribuição do sinal para $M_{Z^{\prime}}=0.5 \mathrm{TeV}$ com $\Gamma_{Z^{\prime}} / M_{Z^{\prime}}=0.1$ e a tracejada está associada ao background $t \bar{t}$.

Podemos obter uma maior redução do background $t \bar{t}$ se analisarmos a distribuição da separação angular azimutal entre os léptons finais, conforme figura (4.8), donde obtemos o corte 


$$
\Delta \phi_{l l^{\prime}}<2.5
$$

Quanto ao caso de léptons de mesmo sabor teremos backgrounds adicionais provenientes da produção de léptons via processos Drell-Yan e do decaimento do $Z Z$. Neste caso devemos ampliar os cortes impondo

$$
\mathbb{E}_{T}>50 \mathrm{GeV}
$$

para suprimir os processo Drell-Yan e

$$
m_{l^{+} l^{-}}>100 \mathrm{GeV}
$$

para reduzir o background $Z Z$.

$\mathrm{Na}$ tabela (4.4) expomos os resultados das seções de choque do background do MP assim como do sinal normalizado como na equação (4.5), para as massas $M_{Z^{\prime}}=0.5,1.0$ e $1.5 \mathrm{TeV}$ no caso especial $\Gamma_{Z^{\prime}}=0.05 M_{Z^{\prime}}$. Analogamente ao caso anterior para as massas e larguras utilizadas para $Z^{\prime}$ o termo $\sigma_{i n t}$ é desprezível.

\begin{tabular}{|c|c|c|c|}
\hline$M_{Z^{\prime}}(\mathrm{TeV})$ & $\sigma_{M P}^{E W}(\mathrm{fb})$ & $\sigma_{M P}^{t t}(\mathrm{fb})$ & $\sigma_{Z^{\prime}}(\mathrm{fb})$ \\
\hline \hline 0.5 & 184 & 10.8 & 793 \\
1.0 & 92.5 & 1.88 & 361 \\
1.5 & 52 & 0 & 158 \\
\hline
\end{tabular}

Tabela 4.4: Seção de choque para o sinal $Z^{\prime}$ e background em fb para o estado final $l^{+} l^{\prime-} \mathbb{E}_{T}\left(l, l^{\prime}=e, \mu\right)$. Para léptons de mesmo sabor foram aplicados os cortes (4.23) - (4.25), já para léptons de sabores distintos (4.23) - (4.28). Nesses resultados ainda não foram incluídas as eficiências experimentais de reconstrução de jatos e léptons. Para o sinal apresentamos os resultados para o valor ilustrativo $\Gamma_{Z^{\prime}}=$ $0.05 M_{Z^{\prime}}$. 


\subsection{3 $p p \rightarrow W^{ \pm} \rightarrow W^{ \pm} Z \rightarrow l^{ \pm} j j \mathbb{E}_{T}$}

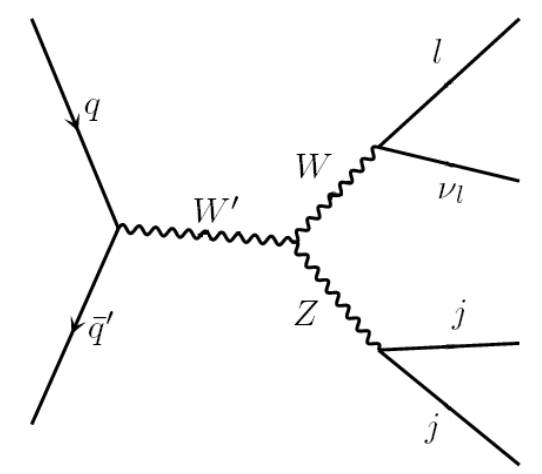

Figura 4.9: Diagrama do sinal $p p \rightarrow W^{ \pm} \rightarrow W^{ \pm} Z \rightarrow l^{ \pm} j j \mathbb{E}_{T}, \operatorname{com} l^{ \pm}=e^{ \pm}, \mu^{ \pm}$.

O sinal do $W^{\prime}$ pode ser visto com o mesmo estado final que utilizamos na busca pelo $Z^{\prime}$ com estado final $l^{ \pm} j j \mathbb{E}_{T}$. O diagrama representativo desse sinal, ilustrado na figura (4.9), indica que agora devemos mudar o corte na massa invariante dos jatos requerendo que $M_{j j}$ seja compatível com $M_{Z}$, todavia $M_{W}$ e $M_{Z}$ possuem massas muito próximas de modo que eventos associados a $W^{\prime}$ podem ser classificados como eventos de $Z^{\prime}$ se estes bósons possuirem massas compatíveis. Neste trabalho assumiremos que estes estados não são degenerados de modo a não termos que levar em conta estas contribuições.

Dado que os sinais $W^{\prime}$ e $Z^{\prime}$ possuem mesmo estado final (mesmo background), podemos utilizar os mesmos cortes (4.18) à (4.22) aplicados ao caso $p p \rightarrow Z^{\prime} \rightarrow$ $W W \rightarrow l^{ \pm} j j \mathbb{E}_{T}$, desde que trocarmos $M_{W}$ por $M_{Z}$ em $(4.20)$ e $M_{Z^{\prime}}$ por $M_{W^{\prime}}$ em $(4.22)$.

\begin{tabular}{|c|c|c|c|c|}
\hline$M_{W^{\prime}}(\mathrm{TeV})$ & $\sigma_{M P}^{E W}(\mathrm{fb})$ & $\sigma_{M P}^{Q C D}(\mathrm{fb})$ & $\sigma_{M P}^{t \bar{t}}(\mathrm{fb})$ & $\sigma_{W^{\prime}}(\mathrm{fb})$ \\
\hline \hline 0.5 & 13.2 & 210. & 11.4 & 1354. \\
\hline 1.0 & 4.0 & 50. & 4.4 & 673. \\
\hline 1.5 & 1.5 & 14. & 0.88 & 32. \\
\hline
\end{tabular}

Tabela 4.5: Seção de choque para o sinal $W^{\prime}$ e background em fb para o estado final $j j l^{ \pm} \mathbb{E}_{T}(l=e, \mu)$ após os cortes (4.18) - (4.22) trocando $M_{W}$ por $M_{Z}$ em (4.20) e $M_{Z^{\prime}}$ por $M_{W^{\prime}}$ em (4.22). Nesses resultados ainda não foram incluídas as eficiências experimentais de reconstrução de jatos e léptons. Para o sinal apresentamos os resultados para o valor ilustrativo $\Gamma_{W^{\prime}}=0.05 M_{W^{\prime}}$.

Na tabela (4.5) apresentamos a seção de choque para o background do MP após aplicarmos os cortes, além da seção de choque para o sinal normalizado como 
em (4.5) para $M_{W^{\prime}}=0.5,1.0$ e $1.5 \mathrm{TeV}$ com $\Gamma_{W^{\prime}}=0.05 M_{W^{\prime}}$. Cabe ressaltar que após aplicarmos os cortes a parte de interferência $\sigma_{i n t}$ fica negligível para os valores utilizados de massas e larguras para o $W^{\prime}$.

\subsection{4 $p p \rightarrow W^{ \pm} \rightarrow W^{ \pm} Z \rightarrow l^{ \pm \pm} l^{+} l^{-} \mathbb{E}_{T}$}

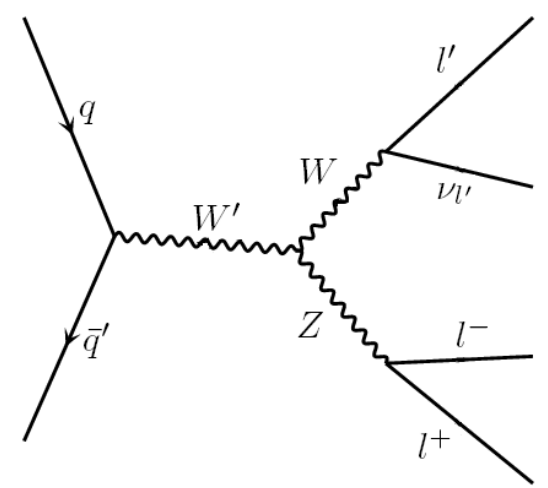

Figura 4.10: Diagrama do sinal $p p \rightarrow W^{ \pm \pm} \rightarrow W^{ \pm} Z \rightarrow l^{+} l^{-} l^{ \pm} \mathbb{E}_{T}, \operatorname{com} l^{ \pm}, l^{ \pm}=$ $e^{ \pm}, \mu^{ \pm}$.

Consideremos o estado final puramente leptônico do processo $p p \rightarrow W^{\prime} \rightarrow W Z$ seguido pelos decaimentos $W \rightarrow l^{\prime} \nu_{l^{\prime}}$ e $Z \rightarrow l l$, onde $l, l^{\prime}=e, \mu$ como denota a figura (4.10). Os backgrounds do MP dominantes originam também da produção de $W Z$ no mesmo estado final e $Z Z$ em léptons carregados com um deles sendo perdido.

Partimos dos cortes básicos para assegurar detecção e isolamento dos léptons do estado final:

$$
\left|\eta_{l}\right|<2.5, \quad \Delta R_{l l}>0.4, \quad \text { e } \quad p_{T}^{l}>10 \mathrm{GeV} .
$$

Visando reduzir ainda mais o background do MP, para a ressonância de menor massa $\left(M_{W^{\prime}}=500 \mathrm{GeV}\right)$ suplementamos que o corte para o momento transversal do lépton mais energético seja maior que $120 \mathrm{GeV}$.

Como no sinal os léptons decaindo do $Z$ possuem mesmo sabor e cargas opostas (MSCO) podemos melhorar a observação do sinal requerendo que a massa invariante desses léptons $M_{l l}^{(M S C O) 2}=\left(p_{l^{+}}+p_{l^{-}}\right)^{2}$ seja compatível com $M_{Z}$

$$
\left|M_{l l}^{(M S C O)}-M_{Z}\right|<20 \mathrm{GeV} .
$$


Dado que o estado final do sinal possui apenas um neutrino podemos reconstruir a massa invatiante $l l l^{\prime} \nu_{l^{\prime}}$ a menos da ambiguidade inerente ao momento longitudinal do neutrino. Deste modo suplementamos ainda os cortes exigindo que a massa reconstruída possua um valor compatível com a massa do $M_{W^{\prime}}=0.5$ (1.0) [1.5] $\mathrm{TeV}$ :

$$
\left|M_{r e c}^{\min }-M_{W^{\prime}}\right|<50,(100),[200] \mathrm{GeV}
$$

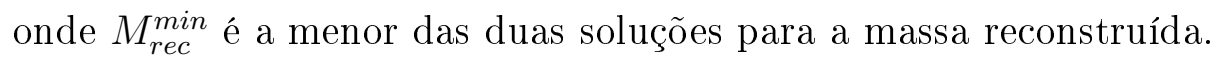

\begin{tabular}{|c|c|c|}
\hline$M_{W^{\prime}}(\mathrm{TeV})$ & $\sigma_{M P}(\mathrm{fb})$ & $\sigma_{W^{\prime}}(\mathrm{fb})$ \\
\hline \hline 0.5 & 0.87 & 297 \\
\hline 1.0 & 0.14 & 85 \\
\hline 1.5 & 0.01 & 6.4 \\
\hline
\end{tabular}

Tabela 4.6: Seção de choque para o sinal e background com estado final $l^{ \pm} l^{+} l^{-} \mathbb{E}_{T}$ $\left(l, l^{\prime}=e, \mu\right)$ após os cortes (4.29) -(4.31). $\sigma_{M P}$ denota a soma das contribuições do background redutível e irredutível. Para o sinal apresentamos os resultados para o valor ilustrativo $\Gamma_{W^{\prime}}=0.05 M_{W^{\prime}}$.

Os resultados para a seção de choque do sinal e background após os cortes (4.29) - (4.31) estão apresentados na tabela (4.6). O termo de interferência como nos casos anteriores é desprezível para o intervalo de massas e larguras adotadas para o $W^{\prime}$. Nesta tabela observamos que o MP possui um background pequeno devido ao pequeno branching ratio do $W Z$ nos léptons do estado final. O sinal além de compartilhar desse pequeno branching ratio ainda possui, como já discutido nas seções anteriores, a seção de choque reduzida para os casos de altos valores de massas para o $W^{\prime}$ que implica em um $Z$ decaindo em léptons muito energéticos e muito colimados não passando pelos cortes de isolamento $\Delta R_{l l}$. 


\subsection{5 $\quad p p \rightarrow W^{ \pm} \rightarrow W^{ \pm} Z \rightarrow l^{+} l^{-} j j$}

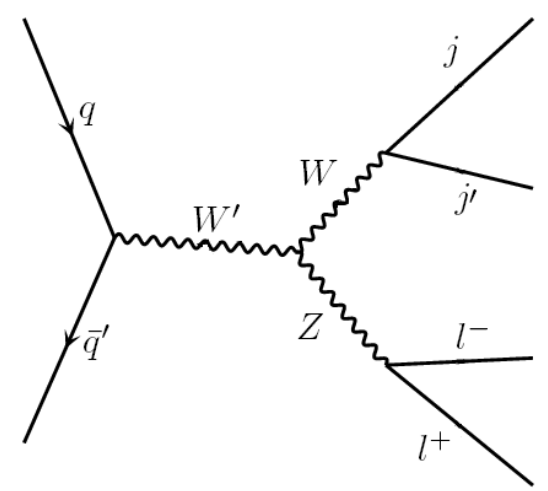

Figura 4.11: Diagrama do sinal $p p \rightarrow W^{ \pm} \rightarrow W^{ \pm} Z \rightarrow l^{+} l^{-} j j, \operatorname{com} l^{ \pm}=e^{ \pm}, \mu^{ \pm}$.

Outro canal semi-leptônico de produção de $W^{\prime}$ que estudamos foi $p p \rightarrow W^{\prime \pm} \rightarrow$ $W^{ \pm} Z \rightarrow l^{+} l^{-} j j$, conforme figura (4.11). Neste caso temos todas as partículas do estado final detectadas de modo que podemos reconstruir completamente o sinal $W^{\prime}$ além dos estados intermediários $W$ e $Z$, possibilitando uma maior redução do background.

$\mathrm{Na}$ análise dos melhores cortes partimos dos cortes fundamentais associados as limitações dos detectores:

$$
\left|\eta_{l}\right|<2.5, \quad\left|\eta_{j}\right|<3 \quad \text { e } \Delta R_{j j(j l)(l l)}>0.4
$$

Novamente o $W^{\prime}$ por possuir uma massa muito alta irá produzir jatos e léptons altamente energéticos que possibilitou obtermos como nos casos anteriores cortes ótimos para supressão do background associados ao $p_{T}$ do estado final:

$$
\begin{aligned}
& p_{T}^{l}>50,(100),[100] \mathrm{GeV} \\
& p_{T}^{j}>70,(100),[100] \mathrm{GeV}
\end{aligned}
$$

para $M_{W^{\prime}}=0.5,(1.0),[1.5] \mathrm{TeV}$. Como já destacamos podemos reconstruir a massa do $W$ decaindo hadronicamente e do $Z$ decaindo leptonicamente de modo a ampliarmos nossos cortes com: 


$$
\begin{array}{r}
\left|M_{j j}-M_{W}\right|<10 \mathrm{GeV} \\
\left|M_{l^{+} l^{-}}-M_{Z}\right|<10 \mathrm{GeV}
\end{array}
$$

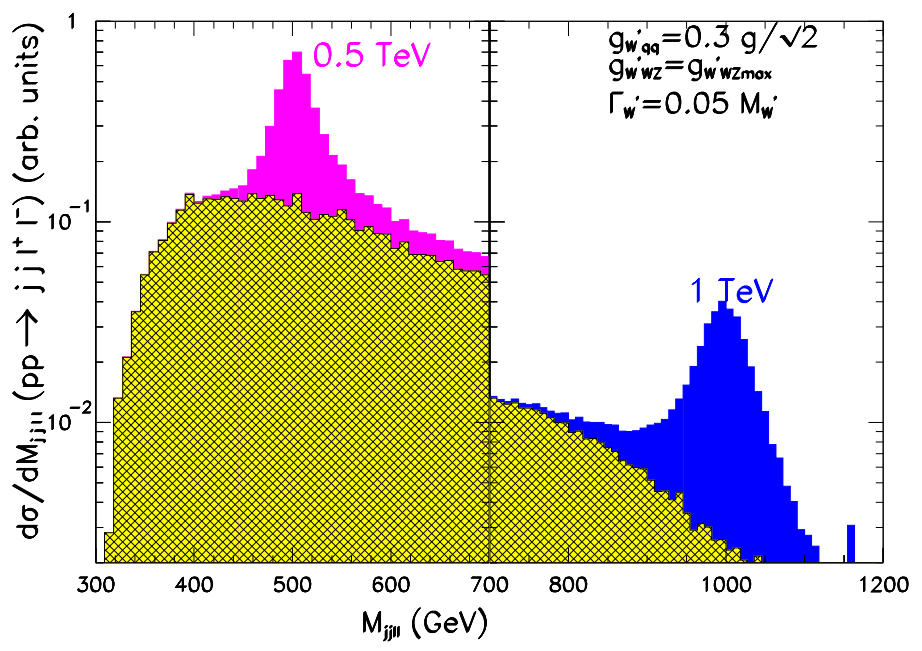

Figura 4.12: Distribuição para a massa invariante reconstruída para o processo $p p \rightarrow l l^{-} j j$ com sinal $M_{W^{\prime}}=0.5 \mathrm{TeV}$ no painel da esquerda e $1.0 \mathrm{TeV}$ no da direita sobre os respectivos backgrounds hachurados, após os cortes (4.32) - (4.34).

Neste caso podemos reconstruir a massa do $W^{\prime}$ sem ambiguidades, pois seu estado final não possui neutrinos. Na figura (4.12) temos a distribuição da massa invariante reconstruída $M_{j j l^{+} l^{-}}$após os cortes (4.32) - (4.34), que indica que podemos melhorar a razão sinal background exigindo que a massa reconstruída seja compatível com $M_{W^{\prime}}=0.5(1.0)[1.5] \mathrm{TeV}$ :

$$
\left|M_{j j l^{+} l^{-}}-M_{W^{\prime}}\right|<50,(100),[200] \mathrm{GeV}
$$

Apresentamos na tabela (4.7) as seções de choque do background MP e sinal após os cortes (4.32) - (4.35). Após os cortes a seção de choque $t \bar{t}$ ficou desprezível frente as demais, o mesmo acontecendo com $\sigma_{\text {int }}$ para as massas e larguras consideradas para o $W^{\prime}$.

Analogamente aos casos anteriores para massas mais altas do $W^{\prime}$ observa-se uma redução mais abrupta do sinal na tabela (4.7), isso se deve aos produtos do $W$ e $Z$ sairem bem colimados não passando pelos cortes de $\Delta R$ (4.32). 


\begin{tabular}{|c|c|c|c|}
\hline$M_{W^{\prime}}(\mathrm{TeV})$ & $\sigma_{M P}^{E W}(\mathrm{fb})$ & $\sigma_{M P}^{Q C D}(\mathrm{fb})$ & $\sigma_{W^{\prime}}(\mathrm{fb})$ \\
\hline \hline 0.5 & 12.2 & 17.2 & 380 \\
\hline 1.0 & 0.14 & 0.76 & 51 \\
\hline 1.5 & 0.03 & 0.12 & 3.3 \\
\hline
\end{tabular}

Tabela 4.7: Sinal do $W^{\prime}$ e background do MP para o estado final $l^{+} l^{-} j j$ com $l^{ \pm}=e^{ \pm}, \mu^{ \pm}$após os cortes (4.32) - (4.35). Nesses resultados ainda não foram incluídas as eficiências experimentais de reconstrução de jatos e léptons. Para o sinal apresentamos os resultados para o valor ilustrativo $\Gamma_{W^{\prime}}=0.05 M_{W^{\prime}}$.

\subsection{Observabilidade do sinal}

Em todos os canais estudados observamos que o termo de interferência $\sigma_{\text {int }}\left(M_{V^{\prime}}, \Gamma_{V^{\prime}}\right)$ $\left(V^{\prime}=W^{\prime}, Z^{\prime}\right)$ apresentou-se desprezível frente as contribuições do MP e do sinal, no intervalo $\Gamma_{V^{\prime}}<M_{V^{\prime}} / 2$ após aplicarmos os cortes. Isto simplifica a análise da significância estatística.

Para um número muito alto de eventos associados ao background $N_{b a c k}$, podemos impor o vínculo de $5 \sigma$ de observabilidade do $V^{\prime}$ assumindo o limite gaussiano:

$$
\begin{gathered}
\frac{N_{\text {sinal }}}{\sqrt{N_{\text {back }}}>5 \Rightarrow \frac{\sigma_{\text {sinal }} \mathcal{L}}{\sqrt{\sigma_{M P} \mathcal{L}}}>5} \\
\Rightarrow\left(\frac{g_{V^{\prime} q \bar{q}}}{g_{V q \bar{q}}} \frac{g_{V^{\prime} W V}}{g_{V^{\prime} W V \max }}\right)^{2}>5 \frac{\sqrt{\sigma_{M P}}}{\sqrt{\mathcal{L} \sigma_{V^{\prime}}\left(M_{V^{\prime}}, \Gamma_{V^{\prime}}\right)}} .
\end{gathered}
$$

onde $N_{\text {sinal }}$ é o número de eventos do sinal e $\mathcal{L}$ é a luminosidade integrada, observe ainda que utilizamos a normalização da seção de choque para o sinal como em (4.5). No que segue adotaremos $\mathcal{L}=100 \mathrm{fb}^{-1}$, com essa luminosidade temos garantido o limite gaussiano para todos os canais dado o alto número de eventos $N_{\text {back }}$. A exceção a esse fato é o canal $p p \rightarrow W^{\prime} \rightarrow W^{ \pm} Z \rightarrow l^{ \pm \pm} l^{+} l^{-} \mathbb{E}_{T}$, dado a baixa seção de choque para o background nesse caso. Neste deve-se adotar o vínculo de $5 \sigma$ de observalidade utilizando a estatística de Poisson.

O vínculo de observabilidade (4.36) não fixa completamente o intervalo do produto $g_{V^{\prime} q \bar{q}} g_{V^{\prime} W V}$, na verdade existe também um vínculo superior de consistência expresso nas equações (4.9) e (4.10), que obtivemos analisando as larguras dos novos bósons vetoriais. Tendo em vista esses limites podemos expressar os intervalos das constantes de acoplamento $g_{V^{\prime} q \bar{q}} g_{V^{\prime} W V}$ em que o sinal seria observado 


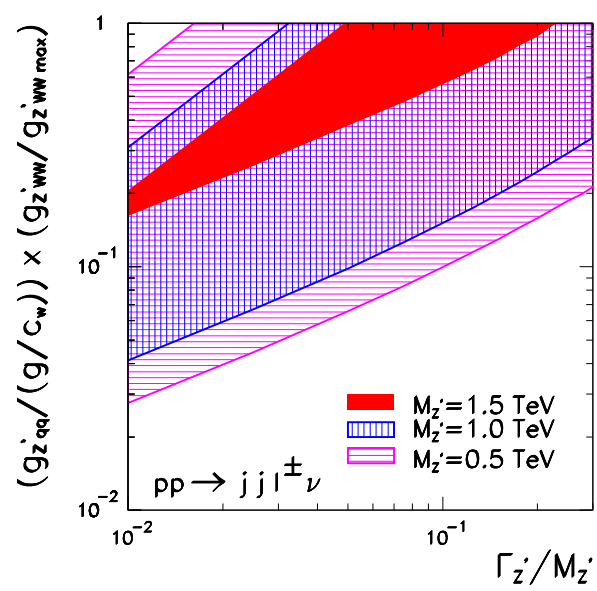

Figura 4.13: As regiões hachuradas referem-se ao intervalo de observação de um $Z^{\prime}$ com massas $M_{Z^{\prime}}=0.5,1.0$ e $1.5 \mathrm{TeV}$ com pelo menos $5 \sigma$ de significância na reação $p p \rightarrow Z^{\prime} \rightarrow W^{+} W^{-} \rightarrow l^{ \pm} j j \mathbb{E}_{T}$.

com pelo menos $5 \sigma$ de observabilidade em função da largura da nova ressonância, que expressamos nas figuras (4.13) - (4.17) para três valores de massas $M_{V^{\prime}}=0.5$, 1.0 e $1.5 \mathrm{TeV}$ para todos canais que estudamos.

Observamos que uma característica compartilhada por todos os canais é crescimento do vínculo inferior em $g_{V^{\prime} q \bar{q}} g_{V^{\prime} W V}$ com a largura total $\Gamma_{V^{\prime}}$. Isso é um simples reflexo de quanto maior a amplitude da largura mais eventos tendem a cair fora do intervalo associado a massa invariante do sinal, veja por exemplo (4.35).

Outra característica é o vínculo inferior do produto dos acoplamentos aumentar com as massas das ressonâncias $M_{V^{\prime}}$. Isso é resultado da combinação do decrescimento da seção de choque para ressonâncias mais massivas e do fato dos produtos serem muito colimados não passando nos cortes de isolamento dos jatos e léptons.

Comparando a observabilidade do $Z^{\prime}$ para o estado final semi-leptônico $l^{ \pm} j j \mathbb{F}_{T}$ figura (4.13) com o puramente leptônico $l^{+} l^{-} \mathbb{E}_{T}$ figura (4.14) constatamos uma melhor observabilidade no canal semi-leptônico com massas $M_{Z^{\prime}}=0.5$ e $1.0 \mathrm{TeV}$ devido a ele possuir um maior branching ratio e por conseguirmos reconstruir o $Z^{\prime}$ a partir do estado final. Para $M_{Z^{\prime}}=1.5 \mathrm{TeV}$ a maior observabilidade é invertida entre esses canais, pois nesse caso os jatos ficam muito colimados não passando nos cortes de isolamento (4.18).

Quanto ao $W^{\prime}$ o melhor canal de observação é o de estado final puramente leptônico $l^{ \pm \pm} l^{+} l^{-} \mathbb{E}_{T}$ devido ao seu pequeno background após os cortes. Observamos também que o estado final $l^{ \pm} j j \mathbb{E}_{T}$ possui uma sensibilidade semelhante entre o 


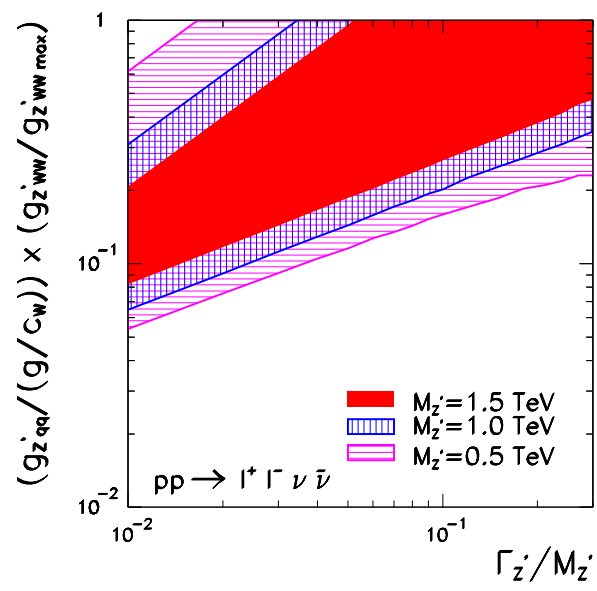

Figura 4.14: As regiões hachuradas referem-se ao intervalo de observação de um $Z^{\prime}$ com massas $M_{Z^{\prime}}=0.5,1.0$ e $1.5 \mathrm{TeV}$ com pelo menos $5 \sigma$ de significância na reação $p p \rightarrow Z^{\prime} \rightarrow W^{+} W^{-} \rightarrow l^{+} l^{-} \mathbb{E}_{T}$.

$W^{\prime}$ e o $Z^{\prime}$, a menos de pequenas diferenças oriundas dos distintos acoplamentos do $W$ e $Z$ com os quarks leves. Além disso esse estado final é um pouco mais sensível que o $l^{+} l^{-} j j$.

Constatamos também que para todas as figuras o limite inferior se aproxima de uma reta para $\Gamma_{V^{\prime}} \lesssim 0.05 M_{V^{\prime}}$ até $0.1 M_{V^{\prime}}$ dependendo do caso. E para larguras maiores foge um pouco desse comportamento linear. Isso é explicado pelo limite de largura fina que diz

$$
\begin{aligned}
\sigma_{\text {sinal }} & \propto \sigma\left(p p \rightarrow V^{\prime}\right) B R\left(V^{\prime} \rightarrow W V\right)+\mathcal{O}\left(\Gamma_{V^{\prime}} / M_{V^{\prime}}\right) \\
& =A \frac{\left(g_{V^{\prime} q \bar{q}} g_{V^{\prime} W V}\right)^{2}}{\Gamma_{V^{\prime}}}+\mathcal{O}\left(\Gamma_{V^{\prime}} / M_{V^{\prime}}\right) .
\end{aligned}
$$

onde $A$ depende apenas de $M_{V^{\prime}}$. Então na aproximação de largura fina o vínculo inferior para o produto das constantes de acoplamento fica escalonado como:

$$
\left(\frac{g_{V^{\prime} q \bar{q}}}{g_{V q \bar{q}}} \frac{g_{V^{\prime} W V}}{g_{V^{\prime} W V \max }}\right)^{2} \times \Gamma_{V^{\prime}}
$$

explicando a forma linear do vínculo inferior para pequenas larguras.

Vale destacar que apesar de o $W^{\prime}$ e $Z^{\prime}$ compartilharem um mesmo estado final $l^{ \pm} j j \mathbb{E}_{T}$, podemos diferenciar essas ressonâncias pelos demais estados finais. O $W^{\prime}$ pode ser identificado pelos sinais adicionais $l^{+} l^{-} j j$ e $l^{\prime \pm} l^{+} l^{-} E_{T}$, já o $Z^{\prime}$ apresentará 


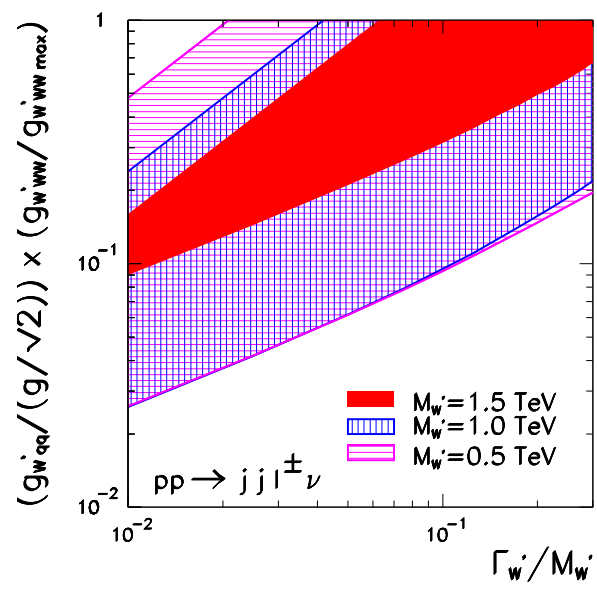

Figura 4.15: As regiões hachuradas referem-se ao intervalo de observação de um $W^{\prime}$ com massas $M_{W^{\prime}}=0.5,1.0$ e $1.5 \mathrm{TeV}$ com pelo menos $5 \sigma$ de significância na reação $p p \rightarrow W^{ \pm} \rightarrow W^{ \pm} Z \rightarrow l^{ \pm} j j \mathbb{E}_{T}$.

também $l^{+} l^{\prime-} \mathbb{E}_{T}$. Neste sentido esses canais são complementares, proporcionando o discernimento das ressonâncias em $W^{\prime}$ e $Z^{\prime}$.

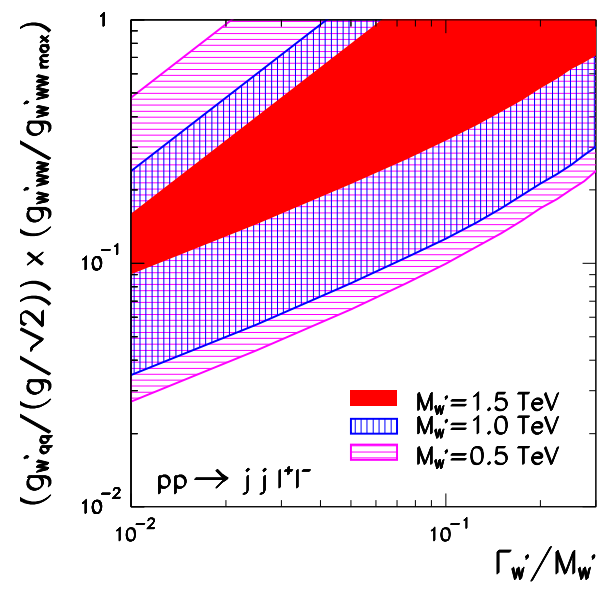

Figura 4.17: As regiões hachuradas referem-se ao intervalo de observação de um $W^{\prime}$ com massas $M_{W^{\prime}}=0.5,1.0$ e $1.5 \mathrm{TeV}$ com pelo menos $5 \sigma$ de significância na reação $p p \rightarrow W^{\prime \pm} \rightarrow W^{ \pm} Z \rightarrow l^{+} l^{-} j j$. 


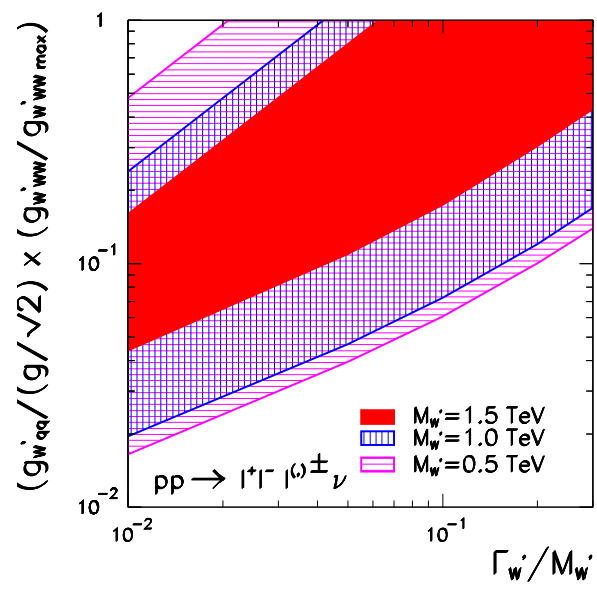

Figura 4.16: As regiões hachuradas referem-se ao intervalo de observação de um $W^{\prime}$ com massas $M_{W^{\prime}}=0.5,1.0$ e $1.5 \mathrm{TeV}$ com pelo menos $5 \sigma$ de significância na reação $p p \rightarrow W^{\prime \pm} \rightarrow W^{ \pm} Z \rightarrow l^{\prime \pm} l^{+} l^{-} \mathbb{E}_{T}$.

\subsection{Conclusões}

Neste capítulo efetuamos uma análise independente de modelo da observabilidade de novos bósons vetoriais, neutros e carregados, no LHC. Essa análise possui como motivação um conjunto bastante amplo de teorias além do MP, as quais buscam a restauração da unitariedade no espalhamento de bósons vetoriais pela inserção de uma torre de Kaluza-Klein de partículas de Spin-1 não requerendo portanto a existência do Higgs.

Com tal motivação analisamos cinco canais de produção dos bósons vetoriais extras $Z^{\prime}$ e $W^{\prime}$ via processos Drell-Yan, equações (4.1) e (4.2). Para esses canais efetuamos uma análise dos melhores cortes para supressão do background de modo que possamos observar o sinais de produção desses novos bósons.

Após encontrados os melhores cortes expressamos um vínculo de observação com $5 \sigma$ desses novos sinais para uma luminosidade de $100 \mathrm{fb}^{-1}$, em que associamos o produto das constantes de acoplamento $g_{V^{\prime} q \bar{q}} g_{V^{\prime} W V}$ com a largura $\Gamma_{V^{\prime}}$ para uma dada massa, expressos nas figuras (4.13) - (4.17) para três valores de massas $M_{V^{\prime}}=$ $0.5,1.0$ e $1.5 \mathrm{TeV}$.

Constatamos na análise desses processos que o LHC cobre uma extensa faixa do espaço de parâmetros dessas teorias com bósons vetoriais extras, permitindo a esse experimento descobrir ou descartar esse tipo de extensão ao MP onde se enquadram os Higgsless Models. 


\section{Capítulo 5}

\section{Conclusão}

Nesta dissertação iniciamos expondo os aspectos teóricos fundamentais do Modelo Padrão e ressaltando sua incrível concordância com os resultados experimentais até a escala de energia provada na atualidade. Apesar desse acordo com os resultados experimentais apontamos a razão da comunidade científica acreditar na existência de nova física já na escala de $1 \mathrm{TeV}$, a ser explorada no LHC, devido ao Problema da Hierarquia de Escalas.

Na tentativa de responder a esse problema tratamos em detalhe do mecanismo de Quebra Espontânea de Simetria por Condições de Contorno em uma dimensão extra compacta, mecanismo fundamental na classe de teorias Além do Modelo Padrão denominada Higgsless Models. Esses modelos postulam a inexistência do Higgs utilizando como mecanismo de restauração da unitariedade no espalhamento entre bósons de gauge, $W W \rightarrow W W$ e $W Z \rightarrow W Z$, uma torre de novos bósons vetoriais que satisfazem as regras de soma obtidas em (3.62) e (3.67). Após a construção de alguns modelos simples no capítulo 3 que possuem apenas carácter didático analisamos um modelo mais realista proposto pela referência [21], que explicita as simetrias que desejavamos proteger em especial a simetria custodial. A partir desse apontamos as modificações aplicadas pelos trabalhos mais recentes para torná-lo uma possível teoria Além do Modelo Padrão, respeitando os vínculos experimentais já estabelecidos.

Essas teorias serviram de inspiração para o capítulo 4, onde efetuamos uma análise independente de modelo da observabilidade de novos bósons vetoriais no LHC associados a restauração da unitariedade, os quais também são previstos pelos Higgsless Models. Analisamos a observabilidade das primeiras novas ressonâncias vetoriais $Z^{\prime}$ e $W^{\prime}$ através cinco canais de produção, equações (4.1) e (4.2). Esses 
canais complementam o trabalho efetuado na referência [29] via fusão de bósons vetoriais, pois por fusão temos apenas a observabilidade do $W^{\prime}$. Deste modo expomos novos modos de análise para o $W^{\prime}$ e apresentamos canais que podem fornecer informações sobre $Z^{\prime}$ que não são obtidas por fusão.

Nossos resultados estão expressos nas figuras (4.13) - (4.17), que apresentam a região do espaço de parâmetros em que o LHC nos proporcionará observação com uma luminosidade integrada de $100 \mathrm{fb}^{-1}$. Essa região está expressa em termos do produto das constantes de acoplamento $g_{V^{\prime} q \bar{q}} g_{V^{\prime} W V}$ e da largura total $\Gamma_{V^{\prime}}$ para três massas $M_{V^{\prime}}=0.5,1.0$ e $1.5 \mathrm{TeV}$.

Dada a vasta região no espaço dos parâmetros coberta com essa luminosidade concluimos que o estudo desses processos nos fornece muita informação, permitindo a descoberta ou exclusão desse conjunto de teorias além do Modelo Padrão que restauram a unitariedade via bósons de Kaluza-Klein. 


\section{Referências Bibliográficas}

[1] C. Quigg. Gauge Theories of Strong, Weak and Eletromagnetic Interactions, Frontier on Physics (1983).

[2] A. Pich, The Standard Model of Electroweak Interactions, arXiv: hepph/0705.4264v1.

[3] C. Burgess; G.Moore, The Standard Model A Primer, Cambridge University Press (2007).

[4] E. A. Paschos, Electroweak Theory, Cambridge University Press (2007).

[5] I. J. R. Aitchison, An Informal Introduction to Gauge Field Theories, Cambridge University Press (1982).

[6] F. Halzen; A. L. Martin. Quarks and Leptons: An Introductory Course in Modern Particle Physics, John Wiley \& Sons (1984).

[7] A. Djouadi, The Anatomy of Electro-Weak Symmetry Breaking, Tome I, Phys. Rept. 457: 1-216 (2008), arXiv: hep-ph/0503172v2.

[8] C. Giunti; C.W. Kim, Fundamentals of Neutrino Physics and Astrophysics, Oxford University Press (2007).

[9] S. Pokorski, Gauge Field Theories 2nd Edition, Cambridge University Press (2000).

[10] C. Amsler et al. (Particle Data Group), Physics Letters B667, 1 (2008).

[11] C. Grojean, Les Houches Summer School, Particle Physics Beyond the Standard Model (2005).

[12] M. Veltman, The Screening Theorem and The Higgs System, Acta Physica Polonica B12, vol. 25 (1994). 
[13] S. Willenbrock, Symmetries of the Standard Model, hep-ph/0410370.

[14] T. Plehn, LHC Physics, http://www.thphys.uni-heidelberg.de/ plehn/.

[15] B. W. Lee, C. Quigg, H. B. Thacker, Phys. Rev. D 16, 1519 (1977).

[16] S. Dawson, Introduction to Eletroweak Symmetry Breaking, AIP Conf. Proc. 1116: 11-34 (2009), arXiv: hep-ph/0812.2190v1.

[17] M. Schmaltz, Physics Beyond the Standard Model: Introducing the Little Higgs, Nucl. Phys. Proc. Suppl. 117 40-49 (2003), arXiv: hep-ph/0210415v2.

[18] C. Kolda; H. Murayama, The Higgs Mass and New Physics Scales in the Minimal Standard Model, JHEP 0007 (2000) 035, arXiv: hep-ph/0003170v1.

[19] LEP Electroweak Working Group, http://www.cern.ch/LEPEWWG/.

[20] C. Csáki; J. Hubisz; P. Meade, TASI Lectures on Eletroweak Symmetry Breaking from Extra Dimensions, arXiv: hep-ph/0510275v1.

[21] C. Csáki; C. Grojean, H. Murayama; L. Pilo; J. Terning, Gauge Theories on an Interval: Unitarity without a Higgs, Phys. Rev. D 69: 055006 (2004), arXiv: hep-ph/0305237v1.

[22] C. Csáki; C. Grojean; L. Pilo; J. Terning, Towards a Realistic Model of Eletroweak Symmetry Breaking, Phys. Rev. Lett. 92: 101802 (2004), arXiv: hep-ph/0308038v2.

[23] T. Gherghetta; A. Pomarol, Bulk fields and supersymmetry in a slice of AdS, Nucl. Phys. B 586: 141-162 (2000), arXiv: hep-ph/0003129v2.

[24] G. Cacciapaglia; C. Csáki; G. Marandella; J. Terning, A New Custodian for Realistic Higgsless Model, Phys. Rev. D 75: 015003 (2007), arXiv: hep$\mathrm{ph} / 0607146 \mathrm{v} 1$.

[25] A. Pérez-Lorenzana, An Introduction to Extra Dimensions, J. Phys. Conf. Ser. 18: 224-269 (2005), arXiv: hep-ph/0503177v2.

[26] G. Cacciapaglia, Higgsless models of eletroweak symmetry breaking, arXiv: hep-ph/0602198. 
[27] R. S. Chivukula, Hong-jian He, M. Kurachi, E. H. Simmons, M. Tanabashi, General Sum Rules for WW Scattering in Higgsless Models: Equivalence Theorem and Deconstruction Identities, Phys. Rev. D 78: 095003 (2008), arXiv: hep-ph/0808.1682v1.

[28] R. S. Chivukula, Lectures on Technicolor and Compositeness, arXiv: hep$\mathrm{ph} / 0011264$.

[29] A. Birkedal, K. Matchev, M. Perelstein, Collider Phenomenology of the Higgsless Models, Phys. Rev. Lett. 94: 191803 (2005), arXiv: hep-ph/0412278v2.

[30] M. Papucci, NDA and perturbativity in Higgsless models, arXiv: hep$\mathrm{ph} / 0408058 \mathrm{v} 1$.

[31] A. Alves, O. J. P. Éboli, D. Gonçalves Netto, M. C. Gonzalez-Garcia, J. K. Mizukoshi, Signals of New Spin-1 Resonances in Eletroweak Gauge Bosons Pais Production at the LHC, arXiv: hep-ph/0907.2915v1.

[32] A. Alves, O. J. P. Éboli, M. C. Gonzalez-Garcia, J. K. Mizukishi, Deciphering the spin o new resonances in Higgsless models, Phys. Rev. D 79: 035009 (2009), arXiv: hep-ph/0810.1952v3.

[33] T. Stelzer and W. F. Long, Comp. Phys. Comm. 81, 357 (1994), arXiv: hep$\mathrm{ph} / 9401258$.

[34] J. Pumplin, D. R. Stump, J. Huston, H. L. Lai, P. Nadolsky and W. K. Tung, JHEP 0207, 012 (2002), arXiv: hep-ph/0201195v3.

[35] K. Agashe et al., LHC Signals for Warped Electroweak Neutral Gauge Bosons, Phys. Rev. D 76: 115015 (2007), arXiv: hep-ph/0709.0007v1.

[36] K. Agashe et al., LHC Signals for Warped Electroweak Charged Gauge Bosons, arXiv: hep-ph/0810.1497v1. 